\title{
Symplectic Forms in the Theory of Solitons*
}

\author{
I.M. Krichever ${ }^{\dagger}$ and D.H. Phong ${ }^{\ddagger}$ \\ † Department of Mathematics \\ Columbia University \\ New York, NY 10027 \\ and \\ Landau Institute for Theoretical Physics \\ Moscow 117940, Russia \\ e-mail: krichev@math.columbia.edu \\ $\ddagger$ Department of Mathematics \\ Columbia University \\ New York, NY 10027 \\ $\ddagger$ e-mail: phong@math.columbia.edu
}

\begin{abstract}
We develop a Hamiltonian theory for 2D soliton equations. In particular, we identify the spaces of doubly periodic operators on which a full hierarchy of commuting flows can be introduced, and show that these flows are Hamiltonian with respect to a universal symplectic form $\omega=\operatorname{Res}_{\infty}\left\langle\Psi_{0}^{*} \delta L \wedge \delta \Psi_{0}\right\rangle d k$. We also construct other higher order symplectic forms and compare our formalism with the case of $1 \mathrm{D}$ solitons. Restricted to spaces of finite-gap solitons, the universal symplectic form agrees with the symplectic forms which have recently appeared in non-linear WKB theory, topological field theory, and Seiberg-Witten theories. We take the opportunity to survey some developments in these areas where symplectic forms have played a major role.
\end{abstract}

${ }^{*}$ Research supported in part by the National Science Foundation under grant DMS-9505399. 


\section{Introduction}

There is increasing evidence that symplectic structures for solitons may provide a unifying thread to many seemingly unrelated developments in geometry and physics. In soliton theory, the space of finite-gap solutions to the equation $\left[\partial_{y}-L, \partial_{t}-A\right]=0$ is a space $\mathcal{M}_{g}(n, m)$ of punctured Riemann surfaces $\Gamma$ and pair of Abelian integrals $E$ and $Q$ with poles of order less than $n$ and $m$ at the punctures. The fibration over $\mathcal{M}_{g}(n, m)$ with $\Gamma$ as fiber carries a natural meromorphic one-form, namely $d \lambda=Q d E$. It is a remarkable and still mysterious fact that the form $d \lambda$ is actually central to several theories with very distinct goals and origins. These include the non-linear WKB (or Whitham) theory [22][23][26][36][38], two-dimensional topological models [11][12][13], and Seiberg-Witten exact solutions of $N=2$ supersymmetric gauge theories [18][40][52][53]. The form $d \lambda$ can be viewed as a precursor of a symplectic structure. Indeed, it can be extended as a 1-form $\sum_{i=1}^{k} d \lambda\left(z_{i}\right)$ on the fibration over $\mathcal{M}_{g}(n, m)$ with fiber a symmetric $g$ th-power of $\Gamma$. Its differential $\omega$ becomes single-valued when restricted to a suitable $g$-dimensional leaf of a canonical foliation on $\mathcal{M}_{g}(n, m)$, and defines a symplectic form [39]. Earlier special cases of this type of construction were pioneered by Novikov and Veselov [50] in the context of hyperelliptic surfaces and 1D solitons, and by Seiberg, Witten, and Donagi [18][53] in the context of N=2 SUSY gauge theories.

The goal of this paper is twofold. Our first and primary objective is to construct the foundations of a Hamiltonian theory of $2 \mathrm{D}$ solitons.

- For this, we provide an improved formulation of 2D hierarchies, since the classical formulations (e.g. Sato [54]) are less pliable than in the 1D case, and inadequate for our purposes. In particular, the new formulation allows us to identify suitable spaces $\mathcal{L}(b)$ of doubly periodic operators on which a full hierarchy of commuting flows $\partial_{m} L=\partial_{y} A_{m}+\left[A_{m}, L\right]$ can be introduced;

- We can then define a universal symplectic form $\omega$ on these spaces $\mathcal{L}(b)$ by

$$
\omega=\operatorname{Res}_{\infty}\left\langle\Psi_{0}^{*} \delta L \wedge \delta \Psi_{0}\right\rangle d k,
$$

where $\Psi_{0}$ and $\Psi_{0}^{*}$ are the formal Bloch and dual Bloch functions for $L$. This form had been shown in [39] to restrict to the geometric symplectic form $\delta \sum_{i=1}^{g} d \lambda\left(z_{i}\right)$ when finite-gap solitons are imbedded in the space of doubly periodic operators. Here we show that it is a symplectic form in its own right on $\mathcal{L}(b)$, and that with respect to this form, the hierarchy of $2 \mathrm{D}$ flows is Hamiltonian. Their Hamiltonians are shown to be $2 n H_{n+m}$, where $H_{s}$ are the coefficients of the expansion of the quasi-momentum in terms of the quasi-energy.

- Our formalism is powerful enough to encompass many diverse symplectic structures for 1D solitons. For example, $\omega$ reduces to the Gardner-FaddeevZakharov symplectic structure for $\mathrm{KdV}$, while its natural modifications for $y$ independent equations (see (2.71) and (2.73) below), reproduce the infinite set of Gelfand-Dickey as well as Adler-Magri symplectic structures. 
- The symplectic form (1.1) is algebraic in nature. However, it suggests new higher symplectic forms,

$$
\omega_{m_{0}}=\operatorname{Res}_{\infty}\left\langle\Psi_{0}^{*}\left(A_{m_{0}}^{(1)} \delta L-L^{(1)} \delta A_{m_{0}}\right) \wedge \delta \Psi_{0}\right\rangle_{0} d k
$$

which are well-defined only on certain spaces of operators with suitable growth or ergodicity conditions. For Lax equations $\partial_{t_{m}} L=\left[A_{m}, L\right]$, these higher symplectic forms have a remarkable interpretation: they are forms with respect to which the eigenvalues of $A_{m_{0}}$, suitably averaged, can serve as Hamiltonians, just as the eigenvalues of $L$ are Hamiltonians with respect to the basic symplectic structure (1.1). It would be very interesting to understand these new forms in an analytic theory of solitons.

Our second objective is to take this opportunity to provide a unified survey of some developments where the form $d \lambda$ (or its associated symplectic form $\omega)$ played a central role. Thus $d \lambda$ emerges as the generating function for the Whitham hierarchy, and its coefficients and periods are Whitham times (Section IV). The same coefficients are deformation parameters of topological Landau-Ginzburg models in two dimensions (Section V), while for $\mathrm{N}=2$ SUSY four-dimensional gauge theories, the periods of $d \lambda$ generate the lattice of Bogomolny-Prasad-Sommerfeld states (Section VI). Together with $d \lambda$, another notion, that of a prepotential $\mathcal{F}$, emerges repeatedly, albeit under different guises. In non-linear WKB methods, $\mathcal{F}$ is the exponential of the $\tau$-function of the Whitham hierarchy. In topological Landau-Ginzburg models, it is the free energy. In $\mathrm{N}=2$ supersymmetric gauge theories, it is the prepotential of the Wilson effective action. It is an unsolved, but clearly very important problem, to determine whether these coincidences can be explained from first principles.

\section{Hamiltonian Theory of 2D Soliton Equations}

Solitons arose originally in the study of shallow water waves. Since then, the notion of soliton equations has widened considerably. It embraces now a wide class of non-linear partial differential equations, which all share the characteristic feature of being expressible as a compatibility condition for an auxiliary pair of linear differential equations. This is the viewpoint we also adopt in this paper. Thus the equations of interest to us are of the form

$$
\left[\partial_{y}-L, \partial_{t}-A\right]=0
$$

where the unknown functions $\left\{u_{i}(x, y, t)\right\}_{i=0}^{n},\left\{v_{j}(x, y, t)\right\}_{j=0}^{m}$ are the $N \times N$ matrix coefficients of the ordinary differential operators

$$
L=\sum_{i=0}^{n} u_{i}(x, y, t) \partial_{x}^{i}, \quad A=\sum_{j=0}^{m} v_{j}(x, y, t) \partial_{x}^{j} .
$$

A preliminary classification of equations of the form (2.1) is by the orders $n, m$ of the operators $L$ and $A$, and by the dimension $N$ of the square matrices 
$u_{i}(x, y, t), v_{j}(x, y, t)$. In what follows, we assume that the leading coefficients of $L$ and $A$ are constant diagonal matrices $u_{n}^{\alpha \beta}=u_{n}^{\alpha} \delta_{\alpha \beta}, v_{m}^{\alpha \beta}=v_{m}^{\alpha} \delta_{\alpha \beta}$. Under this assumption, the equation (2.1) is invariant under the gauge transformations $L, A \rightarrow L^{\prime}=g(x)^{-1} L g(x), A^{\prime}=g(x) A g(x)^{-1}$ where $g(x)$ is a diagonal matrix. We fix the gauge by the condition $u_{n}^{\alpha \beta}=\delta^{\alpha \beta} u_{\alpha}, u_{n-1}^{\alpha \alpha}=0$. We shall refer to (2.1) as a zero curvature or $2 D$ soliton equation. The $1 \mathrm{D}$ soliton equation corresponds to the special case of $y$-independent operators $L$ and $A$. In this case the equation (2.1) reduces to a Lax equation $L_{t}=[A, L]$.

\section{A. Difficulties in a Hamiltonian Theory of 2D Solitons}

The Hamiltonian theory of 1D solitons is a rich subject which has been developed extensively over the years [10][25]. However, much less is known about the $2 \mathrm{D}$ case. We illustrate the differences between $1 \mathrm{D}$ and $2 \mathrm{D}$ equations in the basic example of the hierarchies for the Korteweg-deVries (KdV)

$$
u_{t}-\frac{3}{2} u \partial_{x} u-\frac{1}{4} \partial_{x}^{3} u=0
$$

and the Kadomtsev-Petviashvili (KP) equations

$$
\frac{3}{4} u_{y y}=\partial_{x}\left(u_{t}-\frac{3}{2} u \partial_{x} u-\frac{1}{4} \partial_{x}^{3} u\right) .
$$

The KP equation arises from the choice $N=1, n=2, m=3$, and $L=\partial_{x}^{2}+u$, $A=\partial_{x}^{3}+\frac{3}{2} u \partial_{x}+v_{0}$ in (2.1). We obtain in this way the system

$$
\partial_{x} v_{0}=\frac{3}{4} \partial_{x}^{2} u+\frac{3}{4} u_{y}, \quad v_{0, y}=u_{t}-\frac{1}{4} \partial_{x}^{3} u+\frac{3}{4} \partial_{x} u_{y}-\frac{3}{2} u \partial_{x} u
$$

which is equivalent to (2.4) (up to an $(x, y)$-independent additive term in $v_{0}$, which does not affect the commutator $\left.\left[\partial_{y}-L, \partial_{t}-A\right]\right)$. Taking $L$ and $A$ independent of $y$ gives the $\mathrm{KdV}$ equation.

The basic mechanism behind this construction is that the zero curvature equation actually determines $A$ in terms of $L$. This remains the case for the 1D Lax equation $L_{t}=[A, L]$ even when $A$ is taken to be of arbitrarily high order $m$, but not for the $2 \mathrm{D}$ zero curvature equation $L_{t}-A_{y}=[A, L]$. The point is that $[A, L]$ is a differential operator of order $m+1$. The Lax equation requires that it be in fact of order 0 , while the $2 \mathrm{D}$ zero curvature equation requires only that it be of order $\leq m-1$. The order 0 constraint is quite powerful. Expressed as differential constraints on the coefficients of $A$, it implies readily that the space of such $A$ 's for fixed $L$ is of dimension $m$. An explicit basis can be obtained by the Gelfand-Dickey construction [10][27], which we present for a general operator $L$ of order $n$. Let a pseudo-differential operator of order $n$ be a formal Laurent series $\sum_{i=-\infty}^{n} w_{i} \partial_{x}^{i}$ in $\partial_{x}$, with $\partial_{x}$ and $\partial_{x}^{-1}$ satisfying the identities

$$
\partial_{x} u=u \partial_{x}+u^{\prime}, \partial_{x}^{-1} u=\sum_{i=0}^{\infty}(-)^{i} u^{(i)} \partial_{x}^{-i-1}
$$


Then there exists a unique pseudo-differential operator $L^{1 / n}$ of order 1 satisfying $\left(L^{1 / n}\right)^{n}=L$. Evidently, the coefficients of $L^{1 / n}$ are differential polynomials in the coefficients of $L$. For example, for $L=\partial_{x}^{2}+u$, we find $L^{1 / 2}=\partial_{x}+\frac{1}{2} u \partial_{x}^{-1}-$ $\frac{1}{4} u^{\prime} \partial_{x}^{-2}+\ldots$. We set

$$
L^{i / n}=L_{+}^{i / n}+L_{-}^{i / n}
$$

where the first term on the right hand side is the differential part of the pseudodifferential operator $L^{i / n}$, and the second term on the right hand side is of order $\leq-1$. Then $\left[L, L_{+}^{i / n}\right]=\left[L, L^{i / n}\right]-\left[L, L_{-}^{i / n}\right]=-\left[L, L_{-}^{i / n}\right]$. Since the commutator $\left[L, L_{-}^{i / n}\right]$ is of order at most $n-2$, this shows that the differential operators $L_{+}^{i / n}$ provide the desired basis.

Associated to $L$ are then an infinite hierarchy of flows, obtained by introducing "times" $t_{1}, \ldots, t_{m}, \ldots$, and considering the evolutions of $L=\sum_{i=0}^{n} u\left(x ; t_{1}, \ldots, t_{n}\right) \partial_{x}^{i}$ defined by

$$
\partial_{m} L=\left[L, L_{+}^{m / n}\right]
$$

where we have denoted by $\partial_{m}$ the partial derivative with respect to the time $t_{m}$. A key property of these flows is their commutativity, i.e.

$$
\left[\partial_{i}-L_{+}^{i / n}, \partial_{j}-L_{+}^{j / n}\right]=0 .
$$

To see this, we note first that if $L$ evolves according to a flow $\partial_{t} L=[L, A]$, then $L^{\alpha}$ evolves according to $\partial_{t} L^{\alpha}=\left[L^{\alpha}, A\right]$. Thus we have $\partial_{i} L^{j / n}=\left[L_{+}^{i / n}, L^{j / n}\right]$, $\partial_{j} L^{i / n}=\left[L_{+}^{j / n}, L^{i / n}\right]$, and the left hand side of (2.7) can be rewritten as $-\left[L_{+}^{i / n}, L^{j / n}\right]+\left[L_{+}^{j / n}, L^{i / n}\right]+\left[L_{+}^{i / n}, L_{+}^{j / n}\right]$. If we replace $L_{+}^{i / n}, L_{+}^{j / n}$ by $L^{i / n}-L_{-}^{i / n}$ and $L^{j / n}-L_{-}^{j / n}$, all terms cancel, except for $\left[L_{-}^{i / n}, L_{-}^{j / n}\right]$. This term is however pseudo-differential, of order $\leq-3$, and cannot occur in the left hand side of (2.7), which is manifestly a differential operator.

The flows (2.6) are known to be Hamiltonian with respect to an infinite number of symplectic structures with different Hamiltonians. For example, the $\mathrm{KdV}$ equation itself can be rewritten in two Hamiltonian forms

$$
u_{t}=\partial_{x} \frac{\delta H}{\delta u}, \quad u_{t}=\left(\partial_{x}^{3}+2\left(u \partial_{x}+\partial_{x} u\right)\right) \frac{\delta H^{\prime}}{\delta u}
$$

where the skew-symmetric operators $K=\partial_{x}, K^{\prime}=\partial_{x}^{3}+2\left(u \partial_{x}+\partial_{x} u\right)$ correspond to two different symplectic structures (called respectively the GardnerFaddeev-Zakharov [10][25] and Adler-Magri structures [1][41]), and $H=\frac{1}{4} u^{3}-$ $\frac{1}{8} u_{x}^{2}, H^{\prime}=\frac{1}{8} u^{2}$ are the corresponding Hamiltonians.

The situation for the $2 \mathrm{D}$ zero curvature equation is much less simple, since the arguments narrowing $A$ to an $m$-dimensional space of operators break down. Although formally, we may still introduce the KP hierarchy as $\partial_{m} L=\partial_{y} A_{m}+$ $\left[A_{m}, L\right]$, with $A_{m}$ an operator of order $m$ which should also be viewed as an unknown, this is not a closed system of equations for the coefficients of $L$, as 
it was in the case of the Lax equation. Another way, due to Sato [54], is to introduce the KP hierarchy as a system of commuting flows

$$
\partial_{m} \mathrm{~L}=\left[\mathrm{L}_{+}^{m}, \mathrm{~L}\right]
$$

on the coefficients $\left(v_{i}(x, t)\right)_{i=1}^{i=\infty}$ of a pseudo-differential operator $\mathrm{L}$

$$
\mathrm{L}=\partial+\sum_{i=1}^{\infty} v_{i}(x, t) \partial^{-i}
$$

In this form, the KP hierarchy can be viewed as a completely integrable Hamiltonian system. However, it now involves an infinite number of functions $v_{i}$, and its relation to the original KP equation (which is an equation for a single function of two variables $(x, y))$ requires additional assumptions.

\section{A. Quasi-Energy and Quasi-Momentum}

Our first main task is then to identify the space of differential operators with periodic coefficients on which the $\mathrm{KP}$ equation and its higher order analogues can be considered as completely integrable Hamiltonian systems. Our approach actually applies systematically to general $2 \mathrm{D}$ soliton equations. We present these results at the end of this section, and concentrate for the moment on the simplest case of a differential operator $L$ of order $n$. We begin with the construction of the formal Bloch eigenfunction for two-dimensional linear operators with periodic coefficients.

Theorem 4. Let $L$ be an arbitrary linear differential operator of order $n$ with doubly periodic coefficients

$$
\begin{aligned}
& L=\partial_{x}^{n}+\sum_{i=0}^{n-2} u_{i}(x, y) \partial_{x}^{i}, \\
& u_{i}(x+1, y)=u_{i}(x, y+1)=u_{i}(x, y)
\end{aligned}
$$

Then there exists a unique formal solution $\Psi_{0}(x, y ; k)$ of the equation

$$
\left(\partial_{y}-L\right) \Psi_{0}(x, y ; k)=0,
$$

which satisfies the following properties

(i) $\Psi_{0}(x, y ; k)$ has the form

$$
\Psi_{0}(x, y ; k)=\left(1+\sum_{s=1}^{\infty} \xi_{s}(x, y) k^{-s}\right) e^{\left(k x+k^{n} y+\sum_{i=0}^{n-2} B_{i}(y) k^{i}\right)}
$$

(ii) $\Psi_{0}(0,0 ; k)=1$ 
(iii) $\Psi_{0}(x, y ; k)$ is a Bloch function with respect to the variable $x$, i.e.,

$$
\Psi_{0}(x+1, y, k)=w_{1}(k) \Psi_{0}(x, y, k), \quad w_{1}(k)=e^{k} .
$$

The formal solution $\Psi_{0}(x, y, k)$ is then also a Bloch function with respect to the variable $y$ with a Bloch multiplier $w_{2}(k)$

$$
\begin{aligned}
\Psi_{0}(x, y+1 ; k) & =w_{2}(k) \Psi_{0}(x, y ; k) \\
w_{2}(k) & =\left(1+\sum_{s=1}^{\infty} J_{s} k^{-s}\right) e^{\left.\left(k^{n}+\sum_{i=0}^{n-2} B_{i}(1)\right) k^{i}\right)} .
\end{aligned}
$$

Proof. To simplify the notation, we begin with the proof in the case of $n=2$, with $L=\partial_{x}^{2}+u(x, y)$. The formal solution has then the form

$$
\Psi_{0}(x, y ; k)=\left(1+\sum_{s=1}^{\infty} \xi_{s}(x, y) k^{-s}\right) e^{k x+k^{2} y+B_{0}(y)}
$$

Substituting this formal expansion in the equation $\left(\partial_{y}-L\right) \Psi_{0}(x, y ; k)=0$ gives the following equations for the coefficients $\xi_{s}$

$$
\partial_{y} \xi_{s}+\partial_{y} B_{0} \xi_{s}=2 \xi_{s+1}^{\prime}+\xi_{s}^{\prime \prime}+u \xi_{s}
$$

(Here and henceforth, we also denote derivatives in $x$ by primes.) These equations are solved recursively by the formula:

$$
\begin{aligned}
& \xi_{s+1}(x, y)=c_{s+1}(y)+\xi_{s+1}^{0}(x, y) \\
& \xi_{s+1}^{0}(x, y)=\frac{1}{2} \int_{0}^{x}\left(\partial_{y} \xi_{s}(\tilde{x}, y)-\xi_{s}^{\prime \prime}(\tilde{x}, y)+\left(\partial_{y} B_{0}-u(\tilde{x}, y)\right) \xi_{s}(\tilde{x}, y)\right) d \tilde{x}
\end{aligned}
$$

where $c_{s}(y)$ are arbitrary functions of the variable $y$ with the only requirement that $c_{s}(0)=0$, which is dictated by (ii).

Our next step is to show by induction that the Bloch property (ii), which is equivalent to the periodicity condition

$$
\xi_{s}(x+1, y)=\xi_{s}(x, y)
$$

uniquely defines the functions $c_{s}(y)$. Assume then that $\xi_{s-1}(y)$ is known and satisfies the condition that the corresponding function $\xi_{s}^{0}(x, y)$ is periodic. The first step of the induction, namely the periodicity in $x$ of $\xi_{1}(x, y)$, requires $B_{0}(y)$ to be

$$
B_{0}(y)=\int_{0}^{y} \int_{0}^{1} u\left(x^{\prime}, y^{\prime}\right) d x^{\prime} d y^{\prime}
$$

The choice of the function $c_{s}(y)$ does not affect the periodicity property of $\xi_{s}(x, y)$, but it does affect the periodicity in $x$ of the function $\xi_{s+1}^{0}(x, y)$. In order to make $\xi_{s+1}^{0}(x, y)$ periodic, the function $c_{s}(y)$ should satisfy the equation

$$
\partial_{y} c_{s}=-\int_{0}^{1}\left(\partial_{y} \xi_{s}^{0}(x, y)-\left(\xi_{s}^{0}\right)^{\prime \prime}(x, y)+\left(\partial_{y} B_{0}-u(x, y)\right) \xi_{s}^{0}(x, y)\right) d x
$$


Together with the initial condition $c_{s}(0)=0$, this defines $c_{s}$ uniquely

$$
c_{s}=-\int_{0}^{y} d y^{\prime} \int_{0}^{1}\left(\partial_{y} \xi_{s}^{0}\left(x, y^{\prime}\right)-\left(\xi_{s}^{0}\right)^{\prime \prime}\left(x, y^{\prime}\right)+\left(\partial_{y} B_{0}-u\left(x, y^{\prime}\right)\right) \xi_{s}^{0}\left(x, y^{\prime}\right)\right) d x
$$

and completes the induction step.

We can now establish (2.12), also by induction. Assume that $\xi_{s-1}$ satisfies the relation

$$
\xi_{s-1}(x, y+1)-\xi_{s-1}(x, y)=\sum_{i=1}^{s-1} J_{i} \xi_{s-i-1}(x, y)
$$

where $J_{1}, \ldots, J_{s-1}$ are constants. Then (2.14) implies that

$$
\xi_{s}(x, y+1)-\xi_{s}(x, y)=\sum_{i=1}^{s-1} J_{i} \xi_{s-i}(x, y)+J_{s}=\sum_{i=1}^{s} J_{i} \xi_{s-i}(x, y)
$$

with

$$
J_{s}=c_{s}(y+1)-c_{s}(y)-\sum_{i=1}^{s-1} J_{i} c_{s-i}(y) .
$$

We claim that $J_{s}$ is actually constant. In fact, it follows from (2.14) and (2.19) that

$$
\xi_{s}^{0}(x, y+1)-\xi_{s}^{0}(x, y)=\sum_{i=1}^{s-1} J_{i} \xi_{s-i}^{0}(x, y)=\sum_{i=1}^{s-1} J_{i}\left(\xi_{s-i}(x, y)-c_{s-i}(y)\right)
$$

Thus (2.18) implies that

$$
\begin{gathered}
\partial_{y} c_{s}(y+1)-\partial_{y} c_{s}(y)=-\sum_{i=1}^{s-1} J_{i} \int_{0}^{1}\left(\partial_{y} \xi_{s-i}^{0}-\left(\xi_{s}^{0}\right)^{\prime \prime}+\left(\partial_{y} B_{0}-u\right) \xi_{s}^{0}\right) d x \\
=\sum_{i=1}^{s-1} J_{i}\left(\partial_{y} c_{s-i}-\xi_{s+1}^{0}(1, y)-\xi_{s+1}^{0}(0, y)\right)=\sum_{i=1}^{s-1} J_{i} \partial_{y} c_{s-i}
\end{gathered}
$$

In particular the derivative of $J_{s}$ vanishes. This proves Theorem 1 when $n=2$.

The proof can be easily adapted to the case of general $n$. Let $\xi_{s}^{0}(x, y)$ be the coefficients of the formal series

$$
\Psi^{(0)}(x, y, k)=\frac{\Psi_{0}(x, y, k)}{\Psi_{0}(0, y, k)}=\left(1+\sum_{s=1}^{\infty} \xi_{s}^{0}(x, y, k) k^{-s}\right) e^{k x}
$$

Then $\left(\partial_{y}-L\right) \Psi_{0}=0$ is equivalent to the system of equations

$$
\sum_{l=0}^{n-1} C_{n}^{l}\left(\partial_{x}^{n-l} \xi_{s+l}^{0}\right)+\sum_{i=0}^{n-2} u_{i} \sum_{l=0}^{i} C_{i}^{l}\left(\partial_{x}^{i-l} \xi_{s+l}^{0}\right)=\partial_{y} \xi_{s}^{0}+\sum_{j=-s}^{n-2} b_{j} \xi_{j+s}^{0}, \quad s \geq-n+2
$$


where we have assumed that $\xi_{s}^{0}=0$ for $s<0$, and set $b_{j}=b_{j}(y)$ to be the coefficients of the series

$$
\frac{\partial_{y} \Psi_{0}(0, y, k)}{\Psi_{0}(0, y, k)}=k^{n}+\sum_{j=-\infty}^{n-2} b_{j}(y) k^{j} .
$$

These equations are of the form

$$
n \partial_{x} \xi_{s+n-1}^{0}=b_{-s}+F_{s}\left(\xi_{s^{\prime}}^{0}, b_{s^{\prime \prime}}\right) \text {. }
$$

Here $F$ is a linear combination of the $\xi_{s^{\prime}}^{0}, s^{\prime} \leq s+n-2$ and their derivatives, with coefficients which are themselves linear in $u_{i}$ and $b_{s^{\prime \prime}}, s^{\prime \prime}<s$ (c.f. (2.13)). The equations define recursively $b_{-s}(y)$ and $\xi_{s+n-1}^{0}$. The coefficient $b_{-s}$ follows from the periodicity in $x$ of $(2.23)$

$$
b_{-s}(y)=-\int_{0}^{1} F_{s}\left(\xi_{s^{\prime}}^{0}, b_{s^{\prime \prime}}\right) d x
$$

and the coefficient $\xi_{s+n-1}^{0}$ follows in turn

$$
n \xi_{s+n-1}^{0}=b_{-s}(y) x+\int_{0}^{x} F_{s}\left(\xi_{s^{\prime}}^{0}, b_{s^{\prime \prime}}\right) d x^{\prime} .
$$

We can now integrate (2.22) and find that $\Psi_{0}$ has the form (i) with

$$
B_{i}(y)=\int_{0}^{y} b_{i}(y), \quad i=0, \ldots, n-2 .
$$

We obtain at the same time the Bloch property for $\Psi_{0}$ with respect to $y$ with the Bloch multiplier

$$
w_{2}(k)=\int_{0}^{1}\left(k^{n}+\sum_{i=-\infty}^{n-2} b_{i}(y) k^{i}\right) d y .
$$

The proof of Theorem 1 is complete.

In Theorem 1, we have chosen the simple form $w_{1}(k)=e^{k}$ for the Bloch multiplier in $x$. If we view $x$ as a "space" variable, this identifies the spectral parameter $k$ with the "quasi-momentum" (up to a factor of $i=\sqrt{-1}$ ). The variable $y$ can then be interpreted as a time variable in the Schrödinger-like equation $\left(\partial_{y}-L\right) \Psi_{0}=0$. This identifies (again up to a factor of $i$ ) the logarithm of the second Bloch multiplier $w_{2}(k)$ with the quasi-energy $E(k)$. Alternatively, we may change spectral parameters, and introduce the spectral parameter $K$ as well as the coefficients $\left(\mathcal{E}_{i}\right)_{i=-n+2}^{\infty}$ of the expansion in $k$ of the quasi-energy by

$$
\begin{aligned}
K^{n} & =E(k)=\log w_{2}(k)=k^{n}+\sum_{i=-n+2}^{\infty} \mathcal{E}_{i} k^{-i}, \\
K & =k+O\left(k^{-1}\right) .
\end{aligned}
$$


We observe that a change of spectral parameter of the form $k \rightarrow K=k+O\left(k^{-1}\right)$ transforms a formal series in $k$ of the form (i) in Theorem 1 into another formal series of the same form in $K$. With $K$ as spectral parameter, the second Bloch multiplier $w_{2}(K)$ reduces to $w_{2}(K)=\exp \left(K^{n}\right)$, but the first Bloch multiplier $w_{1}(K)$ becomes non-trivial. The coefficients $\left(H_{s}\right)_{s=1}^{\infty}$ of the expansion in $K$ of its logarithm

$$
w_{1}(K)=e^{k(K)}, \quad k(K)=K+\sum_{s=1}^{\infty} H_{s} K^{-s}
$$

will play an important role in the sequel.

\section{B. Basic Constraints}

The coefficients $\mathcal{E}_{i}$ and $H_{s}$ of the expansions (2.28) and (2.29) of the spectral parameters are uniquely defined by the coefficients $\left(u_{i}\right)_{i=0}^{n-2}$ of the operator $L$, and hence can be considered as functionals on the space of periodic operators. We now restrict ourselves to the subspace $\mathcal{L}(b)$ of operators $L$ satisfying the constraints

$$
b_{i}(y)=b_{i}, 0 \leq i \leq n-2,
$$

where $b_{i}(y)=d B_{i}(y) / d y$, with the functions $B_{i}(y)$ defined by (i) in Theorem 1 , and $b=\left(b_{0}, \ldots, b_{n-2}\right)$ are $(n-1)$ fixed constants. On the space $\mathcal{L}(b)$, the essential singularity in the second Bloch multiplier $w_{2}(k)$ simplifies to $k^{n}+\sum_{i=0}^{n-2} b_{i} k^{i}$ (c.f. (2.12)). Comparing with (2.28), we see that the constraints (2.30) fix the values of the first $(n-1)$ functionals $\mathcal{E}_{-i}=b_{i}$. This is in turn clearly equivalent to fixing the values of the first $(n-1)$ functionals $H_{1}, \ldots, H_{n-1}$ in (2.29). We claim that these constraints can also be expressed under the form

$$
\int_{0}^{1} h_{i}\left(u_{j}\right) d x=H_{s}=\text { const }, \quad i=1, \ldots, n-1
$$

where $h_{s}\left(u_{j}\right), j \geq(n-i-1)$, are universal differential polynomials depending only on $n$. In fact, the constraints (2.30) imply that the essential singularity in the Bloch function $\Psi_{0}(x, y ; k)$ is of the form

$$
k x+\left(k^{n}+\sum_{i=0}^{n-2} b_{i} k^{i}\right) y=K x+K^{n} y+O\left(K^{-1}\right) .
$$

Since the expression exp $\left(O\left(K^{-1}\right)\right)$ contains no essential singularity and can be expanded as a formal series in $K^{-1}$, we have shown that the formal Bloch solution $\Psi_{0}(x, y ; k)$ can be rewritten in terms of the spectral parameter $K$ under the form

$$
\Psi_{0}(x, y, K)=\left(1+\sum_{s=1}^{\infty} \zeta_{s}(x, y) K^{-s}\right) e^{K x+K^{n} y}
$$


Substituting $(2.32)$ in $\left(\partial_{y}-L\right) \Psi_{0}(x, y ; K)=0$ gives for the first $(n-1)$ coefficients $\zeta_{1}, \ldots, \zeta_{n-1}$ a system of ordinary differential equations

$$
\sum_{l=0}^{n-1} C_{n}^{l}\left(\partial_{x}^{n-l} \zeta_{s+l}^{0}\right)+\sum_{i=0}^{n-2} u_{i} \sum_{l=0}^{i} C_{i}^{l}\left(\partial_{x}^{i-l} \zeta_{s+l}^{0}\right)=0, \quad s=-n+2 \ldots, 0
$$

which just coincides with the first equations defining formal eigenfunctions for ordinary differential operators (see [33]). Let $\zeta_{i}\left(x, y ; x_{0}\right)$, be the solution of (2.33) with the normalization $\zeta_{0}=1$ and $\zeta_{i}\left(x_{0}, y ; x_{0}\right)=0$. Then the equations (2.33) define recursively differential polynomials $h_{i}\left(u_{j}(x, y)\right)$ such that

$$
\left.\partial_{x} \zeta_{i}\left(x, y ; x_{0}\right)\right|_{x=x_{0}}=\left.h_{i}\left(u_{j}(x, y)\right)\right|_{x=x_{0}} .
$$

The above left hand side is equal to the first coefficients of the logarithmic derivative of $\Psi_{0}$ at $x=x_{0}$, i.e.

$$
K+\sum_{s=1}^{n-1}\left(\left.\partial_{x} \zeta_{s}\left(x, y ; x_{0}\right)\right|_{x_{0}=x}\right) K^{-s}=\frac{\partial_{x} \Psi_{0}(x, y, K)}{\Psi_{0}(x, y, K)}+O\left(K^{-n}\right) .
$$

Integrating gives the first (constant) coefficients of (2.29) and establishes our claim.

Henceforth we will always assume that $L$ is in the space $\mathcal{L}(b)$ of operators with periodic coefficients $\left(u_{i}\right)_{i=0}^{n-2}$ satisfying either one of the equivalent constraints $(2.30)$ or $(2.31)$.

\section{Commuting Flows}

On the space $\mathcal{L}(b)$ we can now define an infinite set of mutually commuting flows as follows. First, we observe that for any formal series of the form (i) in Theorem 1 , there exists for each integer $m \geq 1$ a unique differential operator $A_{m}$ of the form

$$
A_{m}=\partial_{x}^{m}+\sum_{i=0}^{m-2} u_{i, m}(x, y) \partial_{x}^{i}
$$

which satisfies the condition

$$
\left(A_{m}-K^{m}(k)\right) \Psi_{0}(x, y ; k)=O\left(k^{-1}\right) \Psi_{0}(x, y ; k),
$$

where $K(k)$ is defined by (2.28). Indeed, this condition is equivalent to the following finite system of equations for the coefficients $\left(u_{i, m}\right)$ of $A_{m}$

$$
\sum_{i=0}^{m} u_{i, m} \sum_{l=0}^{i} C_{i}^{l}\left(\partial_{x}^{i-l} \zeta_{s+l}\right)=\zeta_{s+m}, \quad s=-m+2, \ldots, 0 .
$$

This system is triangular, and identifies uniquely the coefficients $u_{i, m}$ as differential polynomials in the first $m-1$ coefficients $\xi_{s}$ of $\Psi_{0}(x, y ; k)$. For example, we find

$$
u_{m-2, m}=-m \zeta_{1}^{\prime}, \quad u_{m-3, m}=-m \zeta_{2}^{\prime}-C_{m}^{2} \zeta_{1}^{\prime \prime} .
$$


Let $L$ be now an operator in $\mathcal{L}(b), \Psi_{0}(x, y ; k)$ its Bloch function, and $A_{m}$ be the operators obtained by the preceding construction. Then

$$
\left[\partial_{y}-L, A_{m}\right] \Psi_{0}(x, y, k)=\left(\partial_{y}-L\right) O\left(k^{-1}\right) \Psi_{0}(x, y, k)=O\left(k^{n-2}\right) e^{K x+K^{n} y}
$$

which implies that the operator $\left[\partial_{y}-L, A_{m}\right]$ has order less or equal to $n-2$. We set

$$
\left[\partial_{y}-L, A_{m}\right]=\partial_{y} A_{m}-\left[L, A_{m}\right]=\sum_{i=0}^{n-2} F_{i, m}(x, y) \partial_{x}^{i} .
$$

The functions $F_{i, m}(x, y)$ are uniquely defined by $u_{j}(x, y)$ and can by expressed in terms of multiple integrals of differential polynomials in $u_{j}$. Thus they can also be expressed as

$$
F_{i, m}(x, y)=\hat{F}_{i, m}(u(x, y)), \quad u=\left(u_{0}, \ldots, u_{n-2}\right) .
$$

where $\hat{F}_{i, m}($.$) is a nonlocal but exact functional of u_{j}(x, y)$.

Theorem 5. The system of equations

$$
\partial_{m} u_{i}=\hat{F}_{i, m}(u) \longleftrightarrow \partial_{m} L=\partial_{y} A_{m}+\left[A_{m}, L\right]
$$

defines an infinite set of commuting flows on the subspace $\mathcal{L}(b)$ of doubly periodic operators.

Since $\hat{F}_{i, m}$ and $A_{m}$ are well-defined functions of $L$, we need only check the commutativity of the flows. For this, we need the following version of the uniqueness of Bloch solutions, namely, that if $\Psi(x, y ; k)$ is a Bloch solution $\left(\Psi(x+1, y ; k)=w_{1}(k) \Psi(x, y ; k)\right)$ of the equation $\left(\partial_{y}-L\right) \Psi=0$ having the form

$$
\Psi_{0}(x, y ; k)=\left(\sum_{s=-N}^{\infty} \xi_{s}(x, y) k^{-s}\right) e^{k x+\left(k^{n}+\sum_{i=0}^{n-2} b_{i} k^{i}\right) y}
$$

then $\Psi(x, y ; k)$ must be given by

$$
\Psi(x, y ; k)=a(k) \Psi_{0}(x, y ; k),
$$

where $a(k)$ is a constant Laurent series $a(k)=\sum_{s=N}^{\infty} a_{s} k^{-s}$. This is because the leading coefficient $\xi_{N}$ in any formal solution $\Psi$ is a constant. As a consequence, if $\Psi(x, y ; k)$ is a Bloch solution, then the ratio $\Psi(x, y ; k) \Psi^{-1}(0,0 ; k)$ is also a solution. This expression has all the properties of, and can be identified with $\Psi_{0}$. Since $\Psi(0,0 ; k)$ is a Laurent series, our assertion follows.

Returning to the proof of Theorem 2, we observe that if $\Psi_{0}$ is the Bloch solution to $(2.22)$ then $\left(\partial_{m}-A_{m}\right) \Psi_{0}$ is also a Bloch solution to the same equation. The preceding uniqueness property implies

$$
\left(\partial_{m}-A_{m}\right) \Psi_{0}=-\tilde{\Omega}_{m}(k) \Psi_{0}, \quad \tilde{\Omega}_{m}(k)=K^{m}+O\left(k^{-1}\right)
$$


for a suitable $(x, y)$ independent Laurent series $\Omega_{m}(k)$. In particular, $\left[\partial_{m}-A_{m}, \partial_{n}-A_{n}\right] \Psi_{0}=O\left(k^{-1}\right) \Psi_{0}$. The last equality implies that $\left[\partial_{m}-A_{m}, \partial_{n}-A_{n}\right]$ is an ordinary differential operator in $x$ of order less than zero. Therefore, it must vanish identically

$$
\left[\partial_{m}-A_{m}, \partial_{n}-A_{n}\right]=0
$$

This establishes the commutativity of the flows. The proof of Theorem 2 is complete.

Conversely, let $u(x, y, t, k), t=\left(t_{1}, t_{2}, \ldots\right)$ be a solution to the hierarchy (2.41). Then there exists a unique formal Bloch solution $\Psi(x, y, t ; K)$ of the equations

$$
\left(\partial_{y}-L\right) \Psi=0, \quad\left(\partial_{m}-A_{m}\right) \Psi=0
$$

having the form

$$
\Psi(x, y, t, K)=\left(1+\sum_{s=1}^{\infty} \zeta_{s}(x, y, t) K^{-s}\right) e^{K x+K^{n} y+\sum_{m} K^{m} t_{m}}
$$

The above expression identifies the original variables $x$ and $y$ with the first and the $n$-th times of the hierarchy,

$$
x=t_{1}, \quad y=t_{n},
$$

respectively. More generally, the preceding results show that for periodic operatos $L$, an equation of the zero curvature form $\left[\partial_{y}-L, \partial_{t}-A\right]=0$ must be equivalent to a pencil of equations for the coefficients of $L$ only. In other words, there must exist constants $c_{i}$ such that

$$
A=\sum_{i=1}^{m} c_{i} A_{i}
$$

and the flow is along the basic times $t_{i}=c_{i} t$, of the hierarchy.

Finally, we point out that for all $n$, the equations of the corresponding hierarchy for $L=L_{n}$ have the same form $\left[\partial_{n}-L_{n}, \partial_{m}-L_{m}\right]=0$, and can be considered as reductions of this system. Our approach to these reductions is to select two particular times which we treat as spatial variables, and to impose periodicity conditions in these variables.

\section{Dual Formal Bloch Solutions}

A key ingredient in our construction of symplectic structures on spaces of periodic operators $L$ is the notion of dual Bloch functions $\Psi_{0}^{*}(x, y ; k)$. In the one-dimensional case, dual Bloch functions were introduced in [7]. In our setup, its main properties are as follows: 
- Let $\Psi_{0}(x, y, k)$ be a formal series of the form (i) in Theorem 1. Then there exists a unique formal series $\Psi_{0}^{*}(x, y ; k)$ of the form

$$
\Psi_{0}^{*}(x, y ; k)=e^{-k x-\left(k^{n}+\sum_{i=0}^{n-2} b_{i} k^{i}\right) y}\left(1+\sum_{s=1}^{\infty} \xi_{s}^{*}(x, y) k^{-s}\right),
$$

such that for all non-negative integers $m$ the equalities

$$
\operatorname{Res}_{\infty}\left(\Psi_{0}^{*}(x, y ; k) \partial_{x}^{m} \Psi_{0}(x, y ; k) d k\right)=0, \quad m=0,1, \ldots
$$

are fulfilled;

- If $\Psi_{0}$ is a Bloch function with Bloch multipliers $w_{i}(k)$, then $\Psi_{0}^{*}(x, y ; k)$ is a Bloch function as well with inverse Bloch multipliers

$$
\Psi_{0}^{*}(x+1, y ; k)=w_{1}^{-1}(k) \Psi_{0}^{*}(x, y ; k), \quad \Psi_{0}^{*}(x, y+1 ; k)=w_{2}^{-1}(k) \Psi_{0}^{*}(x, y ; k),
$$

- If $\Psi_{0}(x, y ; k)$ is a solution to the equation $\left(\partial_{y}-L\right) \Psi_{0}=0$, then the series $\Psi_{0}^{*}(x, y ; k)$ is a solution to the adjoint equation

$$
\Psi_{0}^{*}\left(\partial_{y}-L\right)=0
$$

where the action on the left of a differential operator is defined as a formal adjoint action, i.e. for any function $f^{*}$

$$
\left(f^{*} \partial_{x}^{i}\right)=\left(-\partial_{x}\right)^{i} f^{*}
$$

To see this, we begin by noting that, although each of the factors in (2.51) has an essential singularity, their product is a meromorphic differential and the residue is well-defined. It has the form

$$
\operatorname{Res}_{\infty}\left(\Psi_{0}^{*} \partial_{x}^{m} \Psi_{0} d k\right)=\xi_{m}+\xi_{m}^{*}+g_{m}\left(\xi_{1}, \ldots, \xi_{m-1} ; \xi_{1}^{*}, \ldots, \xi_{m-1}^{*}\right)
$$

where $g_{m}$ is linear in $\xi_{s}^{*}$, in $\xi_{s}$ and their derivatives, $s<m$. The condition (2.51) defines then $\xi_{m}$ recursively as differential polynomials in $\xi_{s}, s=1, \ldots, m$. For example, we have

$$
\xi_{1}^{*}=-\xi_{1}, \quad \xi_{2}^{*}=-\xi_{2}+\xi_{1}^{2}-\xi_{1}^{\prime} .
$$

This shows the existence and uniqueness of $\Psi_{0}^{*}$. Since the second statement is a direct corollary of the uniqueness of $\Psi_{0}^{*}$, we turn to the proof of the last statement. First, we show that if $\Psi^{*}(x, y ; k)$ is a formal series

$$
\Psi^{*}(x, y ; k)=e^{-k x-\left(k^{n}+\sum_{i=0}^{n-2} b_{i} k^{i}\right) y}\left(\sum_{s=-N}^{\infty} \xi_{s}^{*}(x, y) k^{-s}\right),
$$

satisfying the equations (2.51), then there exists a unique degree $N$ ordinary linear differential operator $D$ such that

$$
\Psi^{*}(x, y ; k)=\Psi_{0}^{*}(x, y ; k) D .
$$


Since $\partial_{x}^{i} \Psi_{0}^{*}$ satisfies the equations (2.51), we can find $D$ satisfying the condition

$$
\Psi^{*}(x, y ; k)-\Psi_{0}^{*}(x, y ; k) D=O\left(k^{-1}\right) \Psi_{0}^{*}(x, y ; k) .
$$

The above right hand side has the form (2.55) with $N<0$ and satisfies (2.51). Evaluating the leading term, we find that it must vanish identically.

Let $\Psi_{0}$ be a solution of $\left(\partial_{y}-L\right) \Psi_{0}=0$. Then

$$
\operatorname{Res}_{\infty}\left(\partial_{y} \Psi_{0}^{*} \partial_{x}^{m} \Psi_{0} d k\right)=\partial_{y} \operatorname{Res}_{\infty}\left(\Psi_{0}^{*} \partial_{x}^{m} \Psi_{0} d k\right)-\operatorname{Res}_{\infty}\left(\Psi_{0}^{*} \partial_{x}^{m} L \Psi_{0} d k\right)=0
$$

In particular, there exists a differential operator $\tilde{L}$ such that

$$
\partial_{y} \Psi_{0}^{*}=\Psi_{0}^{*} \tilde{L}
$$

Let $f(x)$ be an arbitrary periodic function on one variable. We have

$$
\left.\partial_{y}\left\langle f(x) \Psi_{0}^{*} \Psi_{0}\right\rangle_{x}=\left\langle f(x) \Psi_{0}^{*}(\tilde{L}+L) \Psi_{0}\right)\right\rangle_{x},
$$

where we have denoted as usual the average value in $x$ of any periodic function $g(x)$ by $\langle g\rangle_{x}$. The above left hand side is of order -1 in $k$. On the other hand, if $L+\tilde{L}$ is not equal to zero and $g_{i}(x, y), 0 \leq i \leq n-2$ are its leading coefficients, then the right hand side is of the form $\left\langle f(x) g_{i}(x, y)\right\rangle_{x} k^{i}+O\left(k^{i-1}\right)$. This implies that $\left\langle f g_{i}\right\rangle_{x}=0$. Since $f$ was arbitrary, we conclude that $g_{i}=0$, establishing the last desired property of dual Bloch functions.

We conclude our discussion of dual Bloch functions with several useful remarks. The first is that the identity

$$
\left\langle\Psi_{0}^{*}(x, y ; k) \Psi_{0}(x, y ; k)\right\rangle_{x}=1 .
$$

holds for any formal series $\Psi_{0}(x, y ; k)$ of the form (i) in Theorem 1 and its dual Bloch series $\Psi_{0}^{*}(x, y ; k)$. Indeed, just as in Section II.C, we can show the existence of a unique pseudo-differential operator $\Phi=1+\sum_{s=0}^{\infty} w_{s}(x, y) \partial_{x}^{-s}$ so that

$$
\Psi_{0}(x, y ; k)=\Phi e^{k x+\left(k^{n}+\sum_{i=0}^{n} b_{i} k^{i}\right) y}
$$

As in [10], this implies

$$
\Psi_{0}^{*}(x, y ; k)=\left(e^{-k x-\left(k^{n}-\sum_{i=0}^{n} b_{i} k^{i}\right) y}\right) \Phi^{-1} .
$$

More precisely, let $Q=\sum_{s=N}^{\infty} q_{s}(x) \partial_{x}^{-s}$ be a pseudo-differential operator. Then we may define its residue $\operatorname{res}_{\partial} Q$ by

$$
\operatorname{res}_{\partial} Q=\operatorname{Res}_{\infty}\left(e^{-k x}\left(Q e^{k x}\right)\right) d k=-q_{1} .
$$

The point is that, while the ring of pseudo-differential operators is not commutative, the residue is, after averaging

$$
\left\langle\operatorname{res}_{\partial}\left(Q_{1} Q_{2}\right)\right\rangle_{x}=\left\langle\operatorname{res}_{\partial}\left(Q_{2} Q_{1}\right)\right\rangle_{x} .
$$


This shows that the series defined by the right hand side of (2.58) satisfies (2.51), and hence must coincide with $\Psi_{0}^{*}$. The desired identity (2.56) is now a direct consequence of the two preceding identities and of the associativity of the left and right actions under averaging.

Secondly, we would like to stress that, although $\Psi_{0}^{*}$ is a Bloch solution of the adjoint equation $\Psi_{0}^{*}\left(\partial_{y}-L\right)=0$, its normalization is different from that used for $\Psi_{0}$. This symmetry may be restored if we introduce

$$
\Psi_{0}^{+}(x, y ; k)=\frac{\Psi_{0}^{*}(x, y ; k)}{\Psi_{0}^{*}(0,0 ; k)} .
$$

The inverse relation is then

$$
\Psi_{0}^{*}(x, y ; k)=\frac{\Psi_{0}^{+}(x, y ; k)}{\left\langle\Psi_{0}^{+} \Psi_{0}\right\rangle_{x}} .
$$

Finally, the definition of the action on the left of a differential operator adopted earlier implies that for any degree $N$ differential operator

$$
D=\sum_{i=0}^{N} w_{i}(x) \partial_{x}^{i}
$$

there exist degree $(N-i)$ differential operators $D^{(i)}$ such that for any pair of functions $f^{+}$and $g$ the equality

$$
\left(f^{*} D\right) g=f^{*}(D g)+\sum_{i=0}^{n} \partial_{x}^{i}\left(f^{*}\left(D^{(i)} g\right)\right)
$$

holds. The set of operators $D^{(i)}$ was introduced in [36]. Of particular interest is of course $D^{(0)}=D$, and the "first descendant" of $D$, namely

$$
D^{(1)}=\sum_{i=0}^{n} i w_{i}(x) \partial_{x}^{i-1} .
$$

\section{E. The Basic Symplectic Structure}

We are now in position to introduce a symplectic structure on the space $\mathcal{L}(b)$ of periodic operators $L$ subject to the constraints (2.30), and to show that the infinite set of commuting flows constructed in Theorem 2 are Hamiltonian.

The main ingredients are the one-forms $\delta L$ and $\delta \Psi_{0}$. The one-form $\delta L$ is given by

$$
\delta L=\sum_{i=0}^{n-2} \delta u_{i} \partial_{x}^{i}
$$

and can be viewed as an operator-valued one-form on the space of operators $L=\partial_{x}^{n}+\sum_{i=0}^{n-2} u_{i} \partial_{x}^{i}$. Similarly, the coefficients of the series $\Psi_{0}$ are explicit 
integro-differential polynomials in $u_{i}$. Thus $\delta \Psi_{0}$ can be viewed as a one-form on the space of operators with values in the space of formal series. More concretely, we can write

$$
\delta \Psi_{0}=\left(\sum_{s=1}^{\infty} \delta \xi_{s} k^{-s}\right) e^{k x+\left(k^{n}+\sum_{i=0}^{n-2} b_{i} k^{i}\right) y}=\left(\sum_{s=1}^{\infty} \delta \zeta_{s} K^{-s}\right) e^{K x+K^{n} y} .
$$

The coefficients $\delta \xi_{s}$ (or $\delta \zeta_{s}$ ) can be found from the variations of the formulae $(2.24),(2.25)$ for $\xi_{s}$, or recursively from the equation

$$
\left(\partial_{y}-L\right) \delta \Psi_{0}=(\delta L) \Psi_{0} .
$$

Let $f(x, y)$ be a function of the variables $x$ and $y$. We denote its mean value by

$$
\langle f\rangle=\int_{0}^{1} \int_{0}^{1} f(x, y) d x d y .
$$

Theorem 6. (a) The formula

$$
\omega=\operatorname{Res}_{\infty}\left\langle\Psi_{0}^{*} \delta L \wedge \delta \Psi_{0}\right\rangle d k
$$

defines a symplectic form, i.e., a closed non-degenerate two-form on the space $\mathcal{L}(b)$ of operators $L$ with doubly periodic coefficients. (b) The form $\omega$ is actually independent of the normalization point $\left(x_{0}=0, y_{0}=0\right)$ for the formal Bloch solution $\Psi_{0}(x, y ; k)$. (c) The flows (2.41) are Hamiltonian with respect to this form, with the Hamiltonians $2 n H_{m+n}(u)$ defined by (2.29).

Proof. We require the following formula, which is a generalization of the wellknown expression for the variation of energy for one-dimensional operators. Let $E(k)$ be the quasi-energy which is defined by (2.28). Its coefficients are nonlocal functionals on the space $\mathcal{L}(b)$ of periodic functions $u_{i}(x, y)$ subject to the constraints (2.30). Then we have

$$
\delta E(k)=\left\langle\Psi_{0}^{*} \delta L \Psi_{0}\right\rangle .
$$

Indeed, from the equation $\left(\partial_{y}-L\right) \Psi_{0}=0$ and (2.53), it follows that

$$
\partial_{y}\left\langle\Psi_{0}^{*} \delta \Psi_{0}\right\rangle_{x}=\left\langle\Psi_{0}^{*} \delta L \Psi_{0}\right\rangle_{x}
$$

Taking the integral over $y$ and using the following monodromy property of $\delta \Psi_{0}$

$$
\delta \Psi_{0}(x, y+1 ; k)=w_{2}(k)\left(\delta \Psi_{0}(x, y ; k)+\delta E(k) \Psi_{0}\right) .
$$

we obtain (2.63).

We begin by checking that the form $\left.\left\langle\Psi_{0}^{*} \delta L \wedge \delta \Psi_{0}\right)\right\rangle_{x}$ is periodic in $y$. The shift of the argument $y \rightarrow y+1$ gives

$$
\left\langle\Psi_{0}^{*} \delta L \wedge \delta \Psi_{0}\right\rangle_{x} \longrightarrow\left\langle\Psi_{0}^{*} \delta L \wedge \delta \Psi_{0}\right\rangle_{x}+\left\langle\Psi_{0}^{*} \delta L \Psi_{0}\right\rangle_{x} \wedge \delta E
$$


The second term on the right hand side can be rewritten as $\delta E \wedge \delta E$ and hence vanishes, due to the skew-symmetry of the wedge product.

Next, we show that (b) is a consequence of the basic constraints defining the space $\mathcal{L}(b)$. Let $\Psi_{1}$ be the formal Bloch solution with the normalization $\Psi_{1}\left(x_{1}, y_{1}, k\right)=1$. Then

$$
\Psi_{1}(x, y ; k)=\Psi_{0}(x, y ; k) \Psi_{0}^{-1}\left(x_{1}, y_{1} ; k\right)
$$

and

$$
\operatorname{Res}_{\infty}\left\langle\Psi_{1}^{*} \delta L \wedge \delta \Psi_{1}\right\rangle d k=\omega-\operatorname{Res}_{\infty}\left(\delta E \wedge \frac{\delta \Psi_{0}\left(x_{1}, y_{1} ; k\right)}{\Psi_{0}\left(x_{1}, y_{1} ; k\right)}\right) d k
$$

In view of the constraints (2.30), we have $\delta E=O\left(k^{-1}\right)$. On the other hand, the second factor in the last term of the above right hand side also has order $O\left(k^{-1}\right)$. The product has therefore order $O\left(k^{-2}\right)$ and its residue equals zero.

To see that $\omega$ is a closed form, we express the operator $L$ as

$$
L=\Phi D \Phi^{-1}+O\left(\partial_{x}^{-1}\right), D=\partial_{x}^{n}+\sum_{i=0}^{n-2} b_{i} \partial_{x}^{i}
$$

which can be done in view of (2.57) and (2.58). Therefore

$$
\omega=\left\langle\operatorname{res}_{\partial}\left(D \Phi^{-1} \delta \Phi \Phi^{-1} \wedge \delta \Phi\right)\right\rangle=-\delta\left\langle\operatorname{res}_{\partial}\left(D \Phi^{-1} \delta \Phi\right)\right\rangle
$$

and $\omega$ is closed.

We turn now to the non-degeneracy of $\omega$ on $\mathcal{L}(b)$. Let $V$ be a vector field such that $\omega\left(V_{1}, V\right)=0$ for all vector fields $V_{1}$. Let $\Psi_{1}=\delta \Psi(V)$ be the evaluation of the one-form $\delta \Psi$ on $V$. Then the equality

$$
\omega\left(V_{1}, V\right)=\operatorname{Res}_{\infty}\left\langle\Psi_{0}^{*} L_{1} \Psi_{1}\right\rangle d k=0
$$

holds for all degree $n-2$ operators $L_{1}=\delta L\left(V_{1}\right)$. Since $L_{1}$ is arbitrary, it follows that $\Psi_{1}=O\left(k^{-n}\right) \Psi_{0}$. In view of $(2.61)$ we have then

$$
\delta L(V) \Psi_{0}=\left(\partial_{y}-L\right) \Psi_{1}=O\left(k^{-1}\right) \Psi_{0} .
$$

Hence $\delta L(V)=0$. This means that $V=0$, and the non-degeneracy of $\omega$ is established.

It remains to exhibit the flows $(2.41)$ as Hamiltonian flows. We recall the classical definition of the Hamiltonian vector field $\partial_{t}$ corresponding to a Hamiltonian $H$ and a two-form $\omega$. The contraction $i\left(\partial_{t}\right) \omega$ of $\omega$ with the vector field $\partial_{t}$ should be the one-form given by the differential of the Hamiltonian, i.e. the equality

$$
i\left(\partial_{t}\right) \omega(X)=\omega\left(X, \partial_{t}\right)=d H(X),
$$

should be fulfilled for all vector-fields $X$. 
The contraction of the form $\omega$ defined by (2.62) with the vector-field $\partial_{m}$ (2.41) is equal to

$$
i\left(\partial_{m}\right) \omega=\operatorname{Res}_{\infty}\left\langle\Psi_{0}^{*} \delta L \partial_{m} \Psi_{0}\right\rangle d k-\operatorname{Res}_{\infty}\left\langle\Psi_{0}^{*}\left(\partial_{m} L\right) \delta \Psi_{0}\right\rangle d k .
$$

Here we use the fact that the evaluations of the forms $\delta L$ and $\delta \Psi_{0}$ on the vector field $\partial_{m}$ are equal by definition to

$$
\delta L\left(\partial_{m}\right)=\partial_{m} L, \quad \delta \Psi_{0}\left(\partial_{m}\right)=\partial_{m} \Psi_{0}
$$

From (2.44) it follows that

$$
\operatorname{Res}_{\infty}\left\langle\Psi_{0}^{*} \delta L \partial_{m} \Psi_{0}\right\rangle d k=\operatorname{Res}_{\infty}\left(\left\langle\Psi_{0}^{*} \delta L A_{m} \Psi_{0}\right\rangle+\tilde{\Omega}_{m}(k)\left\langle\Psi_{0}^{*} \delta L \Psi_{0}\right\rangle\right) d k .
$$

The first term in the right hand side is zero due to the definition of $\Psi_{0}^{*}$. The usual formula for the implicit derivative

$$
\delta E(k) d k=-\delta k(K) d E
$$

implies that the second term is equal to

$$
\begin{aligned}
-\operatorname{Res}_{\infty} \tilde{\Omega}_{m}(K) \delta k(K) d E & =-\operatorname{Res}_{\infty}\left(K^{m}+O\left(K^{-1}\right)\right)\left(\sum_{s=n}^{\infty} \delta H_{s} k^{-s}\right) d K^{n} \\
& =n \delta H_{n+m} .
\end{aligned}
$$

(Recall that $\delta H_{s}=0, s<n$ due to the constraints.) Consider now the second term in the right hand side of (2.68). The equation (2.41) for $\partial_{m} L$ and the defining equations for $\Psi_{0}$ and $\Psi_{0}^{*}$ imply

$$
\operatorname{Res}_{\infty}\left\langle\Psi_{0}^{*}\left(\partial_{m} L\right) \delta \Psi_{0}\right\rangle_{x} d k=\operatorname{Res}_{\infty} \partial_{y}\left(\left\langle\Psi_{0}^{*} A_{m} \delta \Psi_{0}\right\rangle_{x}\right) d k
$$

Therefore,

$$
\int_{0}^{1} \partial_{y}\left\langle\Psi_{0}^{*} A_{m} \delta \Psi_{0}\right\rangle_{x} d y=\left.\delta E(k)\left\langle\Psi_{0}^{*} A_{m} \Psi_{0}\right\rangle_{x}\right|_{y=0}
$$

The equality (2.37) implies

$$
\left\langle\Psi_{0}^{*} A_{m} \Psi_{0}\right\rangle_{x}=K^{m}+O\left(K^{-1}\right) .
$$

Hence, the second term in (2.68) is equal to

$$
\operatorname{Res}_{\infty}\left\langle\Psi_{0}^{*}\left(\partial_{m} L\right) \delta \Psi_{0}\right\rangle d k=-n \delta H_{m+n}
$$

and Theorem 3 is proved. 
Example 1. For $n=2$, the operator $L$ is the second order differential operator of the form $L=\partial_{x}^{2}+u(x, y)$. The space $\mathcal{L}\left(b_{0}\right)$ is the space of periodic functions with fixed mean value in $x$

$$
\langle u\rangle_{x}=H_{1} \rightarrow\langle\delta u\rangle_{x}=0 .
$$

and the symplectic form $\omega$ becomes

$$
\omega=-\left\langle\delta u \wedge \int_{x_{0}}^{x} \delta u d x\right\rangle .
$$

This symplectic form reduces to the Gardner-Faddeev-Zakharov symplectic form when $u(x, y)=u(x)$ is a function of a single variable $x$. In this case the $\mathrm{KP}$ equation reduces to the $\mathrm{KdV}$ equation.

Example 2. For $n=3$, the operator $L$ is the third order differential operator $L=\partial_{x}^{3}+u \partial_{x}+v$. The space $\mathcal{L}\left(b_{0}, b_{1}\right)$ is the space of doubly periodic functions $u=u(x, y), v=v(x, y)$ satisfying the constraints

$$
\langle u\rangle_{x}=\text { const, }\langle v\rangle_{x}=\text { const. }
$$

The symplectic form $\omega$ works out to be

$$
\omega=-\frac{2}{3}\left(\delta u \wedge \int_{x_{0}}^{x} \delta v d x+\delta v \wedge \int_{x_{0}}^{x} \delta u d x\right) .
$$

In the case where $u$ and $v$ are functions of a single variable $x$, this form gives a symplectic structure for the Boussinesq equation hierarchy

$$
u_{t}=2 v_{x}-u_{x x}, v_{t}=v_{x x}-\frac{2}{3} u_{x x x}-\frac{2}{3} u u_{x} .
$$

Note that the usual form of the Boussinesq equation, $u_{t t}+\left(\frac{4}{3} u u_{x}+\frac{1}{3} u_{x x x}\right)_{x}=0$, as an equation in one unknown function $u$, is the result of eliminating $v$ from the above system.

\section{F. Lax Equations}

In this section, we compare the results obtained in our formalism with the one-dimensional case, where the zero curvature equation reduces to the Lax equation, and where there is a rich theory of Hamiltonian structures. It turns out that the symplectic structure constructed above reduces then to the socalled first (or generalized) Gardner-Faddeev-Zakharov symplectic structure. Thus our approach gives a new representation for this structure, as well as a new proof of its well-known properties (c.f. [10][25]). As we shall see below, the second (Adler-Magri) symplectic structure requires a slight modification, which explains why it is special to the one dimensional case and has no analogue in the proposed Hamiltonian theory of two-dimensional systems.

Our construction of the basic symplectic form $\omega$ easily extends to the construction of an infinite sequence of symplectic structures: 
Theorem 7. Let $l$ be any integer $\geq 0$. Then the formula

$$
\omega^{(l)}=\operatorname{Res}_{\infty} E^{l}(k)\left\langle\Psi_{0}^{*} \delta L \wedge \delta \Psi_{0}\right\rangle_{x} d k,
$$

defines a closed two-form on the space $\mathcal{L}\left(H_{1}, \ldots, H_{n l-1}\right)$ of doubly periodic operators $L$, subject to the constraints $H_{s}=$ const, $s=1, \ldots, n l-1$. The equations (2.8) are Hamiltonian with respect to this form, with the Hamiltonians $2 n H_{m+n(l+1)}(u)$ defined by (2.29).

The proof of the theorem is identical to the proof for the basic structure $\omega$. Specializing to the subspace of periodic $L$ with coefficients depending only on $x$, we can easily verify that the symplectic forms $\omega=\omega^{(0)}$ coincide with the generalized Gardner-Faddeev-Zakharov forms.

The construction of the Adler-Magri symplectic structure is less obvious, although formally it has the form (2.71) with $l=-1$ and the residue at infinity is replaced by the residue at $E=0$. Let $L$ be an ordinary linear differential operator of order $n$ with periodic coefficients. Then for generic values of the complex number $E$, there exist $n$ linearly independent Bloch solutions $\Psi_{i}(x, E)$ of the equation

$$
(L-E) \Psi_{i}=0,
$$

with different Bloch multipliers $w_{i}(E)$,

$$
\Psi_{i}(x+1, E)=w_{i}(E) \Psi_{i}(x, E) .
$$

The value $p_{i}(E)=\log w_{i}(E)$ is called the quasi-momentum. Its differential $d p_{i}(E)$ is well-defined. (In our previous formal theory of Bloch solutions, there are also $n$ different solutions corresponding to the same $E$, due to the relation $E=k^{n}+O\left(k^{n-2}\right)=K^{n}$ which defines $k$ and $K$ only up to a root of unity.) We fix the Bloch solutions $\Psi_{j}^{*}$ of the adjoint equation

$$
\Psi_{i}^{*}(L-E)=0
$$

by the condition

$$
\left\langle\Psi_{i}^{*}(x, E) \Psi_{j}(x, E)\right\rangle_{x}=\delta_{i j}
$$

Theorem 8. The formula

$$
\omega^{(-1)}=\sum_{i=1}^{n} R_{i}^{-1}\left\langle\Psi_{i}^{*}(x, 0) \delta L \wedge \delta \Psi_{i}(x, 0)\right\rangle,
$$

where the constants $R_{i}$ are given by

$$
R_{i}=\left\langle\Psi_{i}^{*} L^{(1)} \Psi_{i}\right\rangle
$$

defines a closed two-form on the space of operators $L$ with coefficients depending only on the variable $x$, and obeying the constraints $H_{s}=$ const, $s=1, \ldots, n-1$. The equations (2.8) are Hamiltonian with respect to this symplectic form, with the Hamiltonians $\mathrm{nH}_{m}(u)$ defined by (2.29). 
We observe that for $n=2$, the operator $L^{(1)}$ reduces to $L^{(1)}=2 \partial_{x}$. The expression $\left\langle\Psi_{i}^{*} L^{(1)} \Psi_{i}\right\rangle_{x}$ is then just the Wronskian of two solutions of the Schrödinger equation.

The proof of Theorem 5 is analogous to the proof of Theorem 3. The formula (2.73) can be rewritten in the form

$$
\omega^{(-1)}=-\operatorname{Res}_{0} \frac{d E}{E} \sum_{i=1}^{n} \frac{\left\langle\Psi_{i}^{*}(x, E) \delta L \wedge \delta \Psi_{i}(x, E\rangle\right.}{\left\langle\Psi_{i}^{*}(x, E) L^{(1)} \Psi_{i}(x, E)\right\rangle} .
$$

Due to the summation over $i$, this expression is independent of the labeling of the Bloch functions. Thus on the right hand side, we have the residue of a well-defined function of $E$. The formula we need for the differential of the branch of the quasi-momentum corresponding to the Bloch solution $\Psi(x, E)$ of (2.72) is the following

$$
i d p\left\langle\Psi_{i}^{*}(x, E) L^{(1)} \Psi_{i}(x, E)\right\rangle=d E .
$$

Its proof is identical to the proof in the finite gap theory (see [36]). Consider the differential $d \Psi$ in the variable $E$ of the Bloch function. Then

$$
(L-E) d \Psi=-\Psi d E .
$$

Integrating from $x_{0}$ to $x_{0}+1$ the identity

$$
0=\left(\Psi^{*}(L-E)\right) d \Psi=-\left(\Psi^{*} \Psi\right) d E+\sum_{j=1}^{n} \partial_{x}^{j}\left(\Psi^{*}\left(L^{j} d \Psi\right)\right)
$$

we obtain

$$
d E=i d p\left(\Psi_{i}^{*}\left(x_{0}, E\right) L^{(1)} \Psi_{i}\left(x_{0}, E\right)+\sum_{j=2}^{n} \partial_{x}^{j-1}\left(\Psi^{*}\left(x_{0}, E\right)\left(L^{j} \Psi\left(x_{0}, E\right)\right)\right) .\right.
$$

The desired formula follows after averaging in $x_{0}$ this last identity.

With the formula $(2.75)$ for $d E$ and the analyticity in $E$ for $E \neq 0$ of all relevant expressions, we can, in the computation of the contracted form $i\left(\partial_{m}\right) \omega^{(-1)}$, reduce the residues at $E=0$ to the residue at $E=\infty$ and get the desired result. For example, we have

$$
-\operatorname{Res}_{0} \sum_{i=1}^{n}\left\langle\Psi_{i}^{*} \delta L \partial_{m} \Psi_{i}\right\rangle \frac{d p_{i}(E)}{E}=\operatorname{Res}_{\infty}\left\langle\Psi^{*}(x, K) \delta L \partial_{m} \Psi(x, K)\right\rangle \frac{d K}{E}=n \delta H_{m} .
$$

\section{G. Higher Symplectic Structures in the Two-Dimensional Case}

In this section, we introduce higher Hamiltonian structures which exist in both one and two dimensions. We would like to emphasize that, in contrast with the 
previous results which have basically an algebraic nature, the following results require in general some additional assumptions on the long-time behavior of the solutions to the hierarchy of flows $\partial_{m} L=\partial_{t} A_{m}+\left[A_{m}, L\right]$. In these higher Hamiltonian structures, the role of the quasi-momentum $k(K)$ for the basic structure is assumed by the quasi-momentums $\Omega_{m}(K)$ corresponding to the higher times of the hierarchy.

Our first step is to study in greater detail the quasi-momentums $\Omega_{m}(K)$, corresponding to higher times of the hierarchy. Their "densities" $\tilde{\Omega}_{m}(K)$ made their first appearance in (2.44). They can be re-expressed as

$$
\tilde{\Omega}_{m}(K)=\left.A_{m} \Psi_{0}(x, y ; k)\right|_{x=y=0}=K^{m}+\sum_{s=1}^{\infty} \tilde{\Omega}_{s, m} K^{-s} .
$$

The coefficients $\tilde{\Omega}_{s, m}$ of $\tilde{\Omega}_{m}(k)$ are integro-differential polynomials in the coefficients of the operator $L$. As stated above, they do not depend on $(x, y)$, but they do depend on the times $t$ if the operator $L$ evolves according to the equation (2.41), i.e. $\tilde{\Omega}=\tilde{\Omega}(K, t)$. From (2.44) and (2.45) it follows that

$$
\partial_{i} \tilde{\Omega}_{m}=\partial_{m} \tilde{\Omega}_{i}
$$

Since the coefficients $\tilde{\Omega}_{s, m}$ are independent of the choice of normalization point, they can be considered as functionals on the space of periodic operators $L$.

The subsequent arguments are based on the variational formulas for these functionals, which were found originally in the case of finite-gap solutions in [36]. Following [36], we use the identity

$$
\begin{aligned}
& \sum_{j>1} \partial_{x}^{j-1}\left(\partial _ { m } \left(\Psi_{0}^{*}\left(L^{(j)} \delta \Psi_{0}\right)-\partial_{y}\left(\Psi_{0}^{*}\left(A_{m}^{(j)} \delta \Psi_{0}\right)\right)\right.\right. \\
& =\sum_{j \geq 1} \partial_{x}^{j-1}\left(\left(\Psi_{0}^{*}\left(L^{(j)}\left(\delta A_{m}+\delta \tilde{\Omega}_{m}\right) \Psi_{0}\right)-\left(\Psi_{0}^{*}\left(A^{(j)} \delta L \Psi_{0}\right)\right)\right.\right. \\
& +\sum_{k, j \geq 1} \partial_{x}^{j+k-1}\left(\Psi_{0}^{*}\left(A_{m}^{(k)} L^{(j)}-L^{(k)} A_{m}^{(j)}\right) \delta \Psi_{0}\right)
\end{aligned}
$$

where $L^{(j)}$ and $A^{(j)}$ are the descendants of the operators $L$ and $A$ defined by (2.60). Note that if $L$ and $A_{m}$ satisfy (2.44), then the equality

$$
\partial_{m} L^{(j)}-\partial_{y} A_{m}^{(j)}+\sum_{k}\left[L^{(k)}, A_{m}^{(j-k)}\right]=0
$$

holds. We now average (2.78) first in $x$ and $y$, and then in the normalization point $x_{0}$ (the last averaging eliminates all terms with $j>1$ ). The outcome is the equality

$$
\delta \tilde{\Omega}_{m}(K, t)\left\langle\Psi_{0}^{*} L^{(1)} \Psi_{0}\right\rangle=\left\langle\Psi_{0}^{*}\left(L^{(1)} \delta A_{m}-A_{m}^{(1)} \delta L\right) \Psi_{0}\right\rangle+\partial_{m}\left\langle\Psi_{0}^{*} L^{(1)} \delta \Psi_{0}\right\rangle .
$$


Let $\mathcal{D}_{m_{0}}$, be the space of all periodic operators $L$ which are stationary under the first $m_{0}$-th flow, i.e. which satisfy the condition

$$
\partial_{y} A_{m_{0}}=\left[L, A_{m_{0}}\right]
$$

It should be emphasized that due to (2.77) onto this space the corresponding density of the corresponding quasi-momentum is a constant, i.e. does not depend on the times

$$
\Omega_{m_{0}}(K)=\tilde{\Omega}_{m_{0}}(K) .
$$

The space carrying a higher symplectic structure is a subspace $\mathcal{D}_{m_{0}}^{(I)}$ of $\mathcal{D}_{m_{0}}$, consisting of the stationary operators $L$ satisfying in addition the following higher constraints

$$
\Omega_{m_{0}}=K^{m_{0}}+\sum_{s=1}^{\infty} \Omega_{s, m_{0}} K^{-i}, \quad \Omega_{s, m_{0}}=I_{s}
$$

for a set $I=\left(I_{1}, \ldots, I_{n-1}\right)$ of $(n-1)$ fixed constants. These constraints replace the constraints $(2.30)$ of our previous considerations. The subspace $\mathcal{D}_{m_{0}}^{(I)}$ is invariant with respect to all the other flows corresponding to times $t_{i}$.

Theorem 9. The formula

$$
\omega_{m_{0}}=\operatorname{Res}_{\infty}\left\langle\Psi_{0}^{*}\left(A_{m_{0}}^{(1)} \delta L-L^{(1)} \delta A_{m_{0}}\right) \wedge \delta \Psi_{0}\right\rangle d k
$$

defines a closed two-form on $\mathcal{D}_{m_{0}}^{(I)}$. The restrictions of the equations (2.41) to this space are Hamiltonian with respect to this form, with Hamiltonians $2 n \Omega_{m_{0}, n+m}$.

Remark 1. This statement has an obvious generalization if we replace the stationary condition $(2.81)$ by the condition that $L$ be stationary with respect to a linear combination of the first $m_{0}$ flows, i.e.

$$
\partial_{y} A=[L, A], \quad A=\sum_{i=0}^{m_{0}} c_{i} A_{i} .
$$

Remark 2. For $m_{0}=1$, we have $A_{1}=\partial, A_{1}^{(1)}=1$, and the formula (2.83) becomes identical to $(2.62)$, i.e. $\omega_{1}=\omega$.

The proof of Theorem 6 is identical to that of Theorem 3, after replacing of (2.63) by the formula

$$
\delta \Omega_{m_{0}}(K) d E=\left\langle\Psi_{0}^{*}\left(L^{(1)} \delta A_{m_{0}}-A_{m_{0}}^{(1)} \delta L\right) \Psi_{0}\right\rangle d k,
$$

which is valid on $\mathcal{D}_{m_{0}}$. This formula is itself a direct corollary of (2.80) and (2.75).

As an example, we consider the case $n=2, m_{0}=3$. For $n=2$, the equations (2.41) define the KP hierarchy on the space of periodic functions $u(x, y)$ of two 
variables. For $A=L_{3}$, the condition (2.81) describes the stationary solutions of the original KP equation, i.e. the space of functions described by the equation

$$
3 u_{y y}+\left(6 u u_{x}+u_{x x x}\right)_{x}=0 .
$$

Theorem 6 asserts that, besides of the basic Hamiltonian structure, the restriction of any flow of the KP-hierarchy to the space of functions $u(x, y)$ subject to $(2.85)$ is Hamiltonian with respect to the structure given by $(2.83)$.

In the one-dimensional case, the constraint (2.81) is equivalent to the restriction of the Lax hierarchy on the space of finite-gap solutions. This space is described by the following commutativity condition for the ordinary differential operators $L$ and $A$ of respective degrees $n$ and $m_{0}$

$$
\left[L, A_{m_{0}}\right]=0 .
$$

This condition is equivalent to a system of ordinary differential equations for the coefficients $u_{i}(x)$ of $L$. Theorem 6 asserts that the restriction of the Lax hierarchy to the space of solutions to (2.86) is Hamiltonian with respect to the symplectic form (2.83). In particular, the first flow (which is just a shift in $x$ ) is Hamiltonian. For the $\mathrm{KdV}$ case the corresponding symplectic structure coincides with the stationary Hamiltonian structure found in [5].

Example 3. We return to the case $n=3$ of Example 2, and consider this time operators $A$ of order $m=2$. Thus the operators $L$ and $A$ are given by $L=$ $\partial_{x}^{3}+u \partial_{x}+v, A=\partial_{x}^{2}+\frac{2}{3} u$. The space $\mathcal{D}_{c}$ is the space of two quasi-periodic functions $u(x, y)$ and $v(x, y)$ satisfying the constraints

$$
\langle u\rangle_{x}=\text { const, }\langle v\rangle_{x}=\text { const, }\left\langle u^{2}\right\rangle_{x}=\text { const. }
$$

The operators $L^{(1)}$ and $A^{(1)}$ are given by

$$
L^{(1)}=3 \partial_{x}^{2}+u, \quad A^{(1)}=2 \partial_{x}
$$

and the symplectic form $\omega_{2}$ of $(2.83)$ becomes

$$
\omega=2\left\langle\frac{3}{4} u \delta u \wedge \int_{x_{0}}^{x} \delta u d x+2 \delta v \wedge \int_{x_{0}}^{x} \delta v d x+2 \delta u \wedge \delta v-\frac{3}{2} \delta u_{x} \wedge \delta u\right\rangle .
$$

\section{H. Symplectic Structures under Ergodicity Assumptions}

It may be worthwhile to point out that the existence of the higher Hamiltonian structures obtained in the previous section requires less that the stationary condition (2.81). The only item which was necessary to the argument was the possibility of dropping the last term (which was a full derivative in $t_{m_{0}}$ ) in the formula (2.80).

This suggests considering the space $\mathcal{D}_{m_{0}}^{e r g}$ of all operators $L$ with smooth periodic coefficients, for which the corresponding solution $L\left(t_{m_{0}}\right), L(0)=L$ 
of the equation (2.41) for $m=m_{0}$ exists for all $t_{m_{0}}$ with uniformly bounded coefficients. In this case we may introduce the quasi-momentum

$$
\Omega_{m_{0}}(K)=\lim _{T \rightarrow \infty} \frac{1}{T} \int_{0}^{T} \tilde{\Omega}_{m_{0}}(K, t) d t_{m_{0}} .
$$

Although in this definition, only the dependence on $t_{m_{0}}$ is eliminated through averaging, the quasi-momentum $\Omega_{m_{0}}$ is actually also independent of all the other times $t_{i}$, in view of (2.77).

For $L \in \mathcal{D}_{m_{0}}^{e r g}$ the formula

$$
\delta \Omega_{m_{0}}(K) d E=\left\langle\Psi_{0}^{*}\left(L^{(1)} \delta A_{m_{0}}-A_{m_{0}}^{(1)} \delta L\right) \Psi_{0}\right\rangle_{0} d k
$$

where $\langle f(x, y, t)\rangle_{0}$ stands for

$$
\langle f(x, y, t)\rangle_{0}=\lim _{T \rightarrow \infty} \frac{1}{T} \int_{0}^{T}\langle f(x, y, t)\rangle d t,
$$

holds. Here we make use of the fact that if $\delta L$ is variation of the initial Cauchy data $L(0)$ for $(1.47)$ then the variation $\delta L\left(t_{m_{0}}\right)$ is defined by the linearized equation

$$
\partial_{m_{0}} \delta L-\partial_{y} \delta A_{m_{0}}+\left[\delta L, A_{m_{0}}\right]+\left[L, \delta A_{m_{0}}\right]
$$

With (2.87), it is now easy to establish the following theorem

Theorem 10. The formula

$$
\omega_{m_{0}}=\operatorname{Res}_{\infty}\left\langle\Psi_{0}^{*}\left(A_{m_{0}}^{(1)} \delta L-L^{(1)} \delta A_{m_{0}}\right) \wedge \delta \Psi_{0}\right\rangle_{0} d k,
$$

defines a closed two-form on subspaces of $\mathcal{D}_{m_{0}}^{\text {erg }}$ subject to the constraints (2.82). The restrictions of the equations (2.41) to this space are Hamiltonian with respect to this form, with the Hamiltonians $2 n \Omega_{m_{0}, n+m}$.

The space $\mathcal{D}_{m_{0}}^{\text {erg }}$ appears to be a complicated space, and we do not have at this moment an easier description for it. As noticed in [36], it contains (for an arbitrary $m_{0}$ ) all the finite-gap solutions. There exist a few other cases where we can justify the ergodicity assumption. For example, for the KdV hierarchy, the ergodicity assumption is fulfilled for smooth periodic functions with sufficiently rapidly decreasing Fourier coefficients. Indeed, if $u(x)$ can be extended as an analytic function in a complex neighborhood of real values for $x$ and $y$,

$$
|u(x)|<U, \quad|\operatorname{Im} x|<q
$$

then $u(x, t)$ is bounded by the same constant for all $t$ due to trace formulae. Using the approximation theorem [37] for all periodic solutions to (2.5) (also called the KP-2 equation, by contrast with the KP-1 equation given by (3.19) below) by finite-gap solutions, we can prove the ergodicity assumption in the case when the Fourier coefficients $u_{i j}$ of $u$ satisfy the condition $\left|u_{i j}\right|<U q^{|i|+|j|}$. 
Important Remark. In order to clarify the meaning of the higher symplectic forms and the higher Hamiltonians, it is instructive to explain its analogue for the usual Lax equations. The Lax equations $\partial_{m} L=\left[M_{m}, L\right]$ obviously imply that the eigenvalues of $L$ are integrals of motion, and usually they serve as Hamiltonians for the basic symplectic structure. The higher Hamiltonians correspond to the eigenvalues of the operator $M_{m_{0}}$ instead of $L$. Of course, they are time dependent, but after averaging with respect to one of the times, namely $t_{m_{0}}$, they become nonlocal integrals of motion and can serve as Hamiltonians for the corresponding symplectic structures.

\section{The Matrix Case and the 2D Toda Lattice}

Our formalism extends without difficulty to a variety of more general settings. We shall discuss briefly the specific cases of matrix equations and of the Toda lattice, which correspond respectively to the cases where $L$ is matrix-valued, and where the differential operator $\partial_{x}$ is replaced by a difference operator.

Let $L=\sum_{i=0}^{n} u_{i}(x, y) \partial_{x}^{i}$ be then an operator with matrix coefficients $u_{i}=$ $\left(u_{i}^{\alpha \beta}\right)$ which are smooth and periodic functions of $x$ and $y$, whose leading term $u_{n}^{\alpha \beta}=u_{n}^{\alpha} \delta_{\alpha \beta}$ is diagonal with distinct diagonal elements $u_{n}^{\alpha} \neq u_{n}^{\beta}$ for $\alpha \neq \beta$, and which satisfies $u_{n-1}^{\alpha \alpha}=0$. Then, arguing as in Section II.B, we can show that there exists a unique matrix formal solution $\Psi_{0}=\left(\Psi_{0}^{\alpha \beta}(x, y ; k)\right)$ of the equation $\left(\partial_{y}-L\right) \Psi_{0}=0$, which has the form

$$
\Psi_{0}(x, y ; k)=\left(I+\sum_{s=1}^{\infty} \xi_{s}(x, y) k^{-s}\right) \exp \left(k x+u_{n} k^{n} y+\sum_{i=0}^{n-2} B_{i}(y) k^{i}\right)
$$

(where $I$ is the identity matrix, $\xi_{s}=\left(\xi_{s}^{\alpha \beta}\right)$ are matrix functions, and $B_{i}(y)=$ $\left(B_{i}^{\alpha \beta}(y)\right)=\left(B_{i}^{\alpha}(y) \delta_{\alpha \beta}\right)$ are diagonal matrices $)$, and which has the Bloch property

$$
\Psi_{0}(x+1, y ; k)=\Psi_{0}(x, y ; k) w_{1}(k), w_{1}(k)=e^{k} .
$$

The formal solution $\Psi_{0}(x, y ; k)$ has the Bloch property with respect to $y$ as well,

$$
\Psi_{0}(x, y+1 ; k)=\Psi_{0}(x, y ; k) w_{2}(k)
$$

with the Bloch multiplier $w_{2}(k)$ of the form

$$
w_{2}(k)=\left(1+\sum_{s=1}^{\infty} J_{s} k^{-s}\right) \exp \left(u_{n} k^{n}+\sum_{i=0}^{n-2} B_{i} k^{i}\right)
$$

where $J_{s}$ and $B_{i}$ are diagonal matrices.

As noted at the end of Section II.B, the second Bloch multiplier defines the quasi-energy $E(k)$. This defines in turn the functionals $\mathcal{E}_{i}$ just as in (2.28), with the only difference the fact that they are now diagonal matrices. If we introduce the diagonal matrix $K$ by the equality

$$
u_{n} K^{n}=E(k)=\log w_{2}(k)=u_{n} k^{n}+\sum_{i=-n+2}^{\infty} \mathcal{E}_{i} k^{-i}
$$


then we may define diagonal matrices $H_{s}=\left(H_{s}^{\alpha} \delta_{\alpha \beta}\right)$ by

$$
k I=K+\sum_{i=1}^{\infty} H_{s} K^{-s}
$$

The definition of the commuting flows in the matrix case is then just the same as in the scalar case. The only difference is that the number of these flows is now $N$ times larger. The corresponding times are denoted by $t=\left(t_{\alpha, m}\right)$, and the flows are given by

$$
\left[\partial_{y}-L, \partial_{\alpha, m}-L_{\alpha, m}\right]=0
$$

where $L_{\alpha, m}$ is the unique operator of the form

$$
L_{\alpha, m}=v_{\alpha} \partial_{x}^{m}+\sum_{i=1}^{m-1} u_{i,(\alpha, m)}(t) \partial_{x}^{i}, v_{\alpha}^{\beta \gamma}=\delta_{\alpha \beta} \delta^{\beta \gamma}
$$

which satisfies the condition

$$
L_{\alpha, m} \Psi_{0}(x, y ; k)=\Psi_{0}(x, y ; k)\left(v_{\alpha} K^{m}+O\left(K^{-1}\right)\right) .
$$

As before, the dual Bloch formal series $\Psi_{0}^{*}(x, y ; k)$ is defined as being of the form (2.58), and satisfying the equation

$$
\operatorname{Res}_{\infty} \operatorname{Tr}\left(\Psi_{0}^{*} v \partial_{x}^{m} \Psi_{0}\right) d k=0, m \geq 0
$$

for arbitrary matrices $v$. We have then

Theorem 11. The formula

$$
\omega=\operatorname{Res}_{\infty} \operatorname{Tr}\left\langle\Psi_{0}^{*} \delta L \wedge \delta \Psi_{0}\right\rangle d k
$$

defines a closed non-degenerate two-form on the space of periodic operators $L$ subject to the constraints $H_{s}^{\beta}=$ constant $\beta=1, \ldots, l, s=1, \ldots, n-1$. The equations (2.93) are Hamiltonian with respect to this form, with Hamiltonians $2 n H_{m+n}^{\alpha}$ defined by (2.92).

Example 4. Consider the case $n=1$, where the operator $L$ is of the form $L=A \partial_{x}+u(x, y)$, with $A$ the $N \times N$ matrix $A^{\alpha, \beta}=a_{\alpha} \delta_{\alpha, \beta}$ and $u(x, y)$ is an $N \times N$ matrix with zero diagonal entries $u^{\alpha, \alpha}=0$. In this case the symplectic form (2.96) becomes

$$
\omega=\sum_{\alpha \neq \beta} \frac{1}{a_{\alpha}-a_{\beta}} \delta u^{\beta, \alpha} \wedge \delta u^{\alpha, \beta} .
$$

Finally, as a basic example of a system corresponding to an auxiliary linear equation where the differential operator $\partial_{x}$ is replaced by a difference operator acting on spaces of infinite sequences, we consider the $2 D$ Toda lattice. 
The 2D Toda lattice is the system of equations for the unknown functions $\varphi_{n}=\varphi_{n}\left(t_{+}, t_{-}\right)$

$$
\frac{\partial^{2}}{\partial t_{+} \partial t_{-}} \varphi_{n}=e^{\varphi_{n}-\varphi_{n-1}}-e^{\varphi_{n+1}-\varphi_{n}}
$$

It is equivalent to the compatibility conditions for the following auxiliary linear problem

$$
\partial_{+} \psi_{n}=\psi_{n+1}+v_{n} \psi_{n}, v_{n}=\partial_{+} \varphi_{n}, \partial_{-} \psi_{n}=c_{n} \psi_{n-1}, c_{n}=e^{\varphi_{n}-\varphi_{n-1}} .
$$

We consider solutions of this system which are periodic in the variables $n$ and $y=\left(t_{+}+t_{-}\right)$. The relevant linear operator is the difference operator

$$
L \Psi_{n}=\Psi_{n+1}+v_{n} \Psi_{n}+c_{n} \Psi_{n-1}
$$

with periodic coefficients $v_{n}(y)=v_{n+N}(y)=v_{n}(y+l)$ and $c_{n}(y)=c_{n+N}(y)=$ $c_{n}(y+1)$. Then, arguing as in Section II.B we can show that there exist unique formal solutions $\Psi^{( \pm)}=\Psi_{n}^{( \pm)}(y ; k)$ of the equation

$$
\left(\partial_{y}-L\right) \Psi^{( \pm)}=0
$$

which have the form

$$
\Psi_{n}^{( \pm)}(y ; k)=k^{ \pm n}\left(\sum_{s=0}^{\infty} \xi_{s}^{( \pm)}(n, y) k^{-s}\right) e^{k y+B(y)}, \quad \xi_{0}^{(+)}=1
$$

the Bloch property

$$
\Psi_{n+N}^{( \pm)}(y ; k)=\Psi_{n}^{( \pm)}(y ; k) w_{1}^{( \pm)}(k), \quad w_{1}^{( \pm)}(k)=k^{ \pm N},
$$

and which are normalized by the condition

$$
\Psi_{0}^{( \pm)}(0 ; k)=1
$$

The coefficients $\xi_{s}^{( \pm)}$can be found recursively. The initial value $\xi_{0}^{(+)}=1$ and the condition that $\xi_{1}^{(+)}$is periodic in $n$ define the function $B(y)$ in $(2.97)$

$$
B(y)=N^{-1} \int_{0}^{y}\left(\sum_{n=1}^{N} v_{n}\right) d y
$$

The only difference with the previous differential case is the definition of the leading term $\xi_{0}^{(-)}(n, y)$. Let us introduce $\varphi_{n}(y)=\log \xi_{0}^{(-)}(n, y)$. Then we have

$$
c_{n}(y)=e^{\varphi_{n}(y)-\varphi_{n-1}(y)} .
$$

The periodicity condition for $\xi_{1}^{(-)}$requires the equality

$$
\sum_{n=0}^{N-1} \partial_{y} \varphi_{n}=0
$$


which allows us to define $\varphi_{n}$ uniquely through $c_{n}$ :

$$
\varphi_{n}=\sum_{n=1}^{n-1} \log c_{n}-\int_{0}^{y}\left(N^{-1} \sum_{n=0}^{N-1} \log c_{n}\right) d y .
$$

The formal solutions $\Psi_{n}^{( \pm)}(y, k)$ have the Bloch property with respect to $y$ as well,

$$
\Psi_{n}^{( \pm)}(y+1 ; k)=\Psi_{n}^{( \pm)}(y ; k) w_{2}^{( \pm)}(k)
$$

with the Bloch multipliers $w_{2}^{( \pm)}(k)$ of the form

$$
w_{2}^{( \pm)}(k)=e^{k+B}\left(\sum_{s=0}^{\infty} J_{s}^{( \pm)} k^{-s}\right)
$$

As noted at the end of Section II.B, the second Bloch multiplier defines the quasi-energy $E^{( \pm)}(k)$ and the functionals $\varepsilon_{i}^{( \pm)}$just as in (2.28). If we introduce the variable

$$
K=E^{( \pm)}(k)=\log w_{2}^{( \pm)}(k)=k+\sum_{i=0}^{\infty} \mathcal{E}_{i}^{( \pm)} k^{-i}
$$

then we may define the functionals $H_{s}^{( \pm)}$by

$$
\log k=\log K \pm \sum_{s=0} H_{s}^{( \pm)} K^{-s}
$$

The definition of the commuting flows in the discrete case is then the same as in the scalar case. The basic constraints that specify the space of periodic functions $v_{n}$ and $c_{n}$ have the form

$$
I_{0}=\sum_{n=1}^{n} \log c_{n}(y)=\text { const, } \quad I_{1}=\sum_{n=1}^{N} v_{n}(y)=\text { const. }
$$

The corresponding times are denoted by $t=\left(t_{ \pm, m}\right)$ and the flows are given by

$$
\left[\partial_{y}-L, \partial_{ \pm, m}-L_{ \pm, m}\right]=0
$$

where $L_{ \pm, m}$ is the unique operator of the form

$$
L_{ \pm, m} \Psi_{n}=\sum_{i=0}^{m} u_{i,( \pm, m)}(n, t) \Psi_{n \pm i}, u_{i,(+, m)}=1, u_{i,(-, m)}(n, t)=e^{\varphi_{n}-\varphi_{n-i}}
$$

which satisfies the condition

$$
L_{ \pm, m} \Psi_{n}^{( \pm)}=\Psi_{n}^{( \pm)}\left(K^{m}+O\left(K^{-1}\right)\right) .
$$


The dual formal series $\Psi_{n}^{*,( \pm)}$ are defined as the formal series of the form

$$
\Psi_{n}^{*,( \pm)}(y, k)=k^{\mp n}\left(\sum_{s=0}^{\infty} \xi_{s}^{*,( \pm)}(n, y) k^{-s}\right) e^{-(k y+B(y))}, \quad \xi_{0}^{*,(+)}=1,
$$

which satisfy the equations

$$
\left.\operatorname{Res}_{\infty}\left(\Psi_{n}^{*,(+)} \Psi_{m}^{(+)}\right)-\Psi_{n}^{*,(-)} \Psi_{m}^{(-)}\right) \frac{d k}{k}=0, m \in \mathbf{Z}
$$

for all integers $m$.

Theorem 12. The formula

$$
\omega=\operatorname{Res}_{\infty}\left\langle\Psi^{*,(+)} \delta L \wedge \delta \Psi^{(+)}-\Psi^{*,(-)} \delta L \wedge \delta \Psi^{(-)}\right\rangle \frac{d k}{k}=\left\langle\delta\left(2 v_{n}-\partial_{y} \varphi_{n}\right) \wedge \delta \varphi_{n}\right\rangle
$$

defines a closed non-degenerate two-form on the space of periodic operators $L$ subject to the constraints (2.99). The equations (2.100) are Hamiltonian with respect to this form, with Hamiltonians $H_{m}^{ \pm}$defined by (2.98).

Finally, we would like to conclude this section by calling the reader's attention to [59][60], where symplectic structures are discussed from a group theoretic viewpoint, and where applications of soliton theory to harmonic maps are given.

\section{Geometric Theory of 2D Solitons}

In Section I, we had developed a general Hamiltonian theory of 2D solitons. The central notion was the symplectic form (1.1), which was defined on the infinite-dimensional space $\mathcal{L}(b)$ of doubly periodic operators obeying suitable constraints. Our main goal in this section is to present and extend the results of [39]. In this work, as described in the Introduction, a natural symplectic form $\omega_{\mathcal{M}}$ was constructed on Jacobian fibrations over the leaves of moduli spaces $\mathcal{M}_{g}(n, m)$ of finite-gap solutions to soliton equations. Imbedded in the space of doubly periodic functions, the form $\omega_{\mathcal{M}}$ was shown to coincide with $\omega$ (this was in fact our motivation for constructing a general Hamiltonian theory based on $\omega$ in this paper). Although the infinite-dimensional symplectic form $\omega$ and its variants in Section II can be expected to play an important role in an analytic theory of solitons, it is the geometric and finite-dimensional form $\omega_{\mathcal{M}}$ which has provided a unifying theme with topological and supersymmetric field theories.

In Section II, we have seen how a differential operator $L$ determined a Bloch function $\Psi_{0}$, which was a formal series in a spectral parameter $k$ or $K$. The key to the construction of finite-gap solutions of soliton equations is the reverse process, namely the association of an operator $L$ to a series of the form of $\Psi_{0}$. To allow for evolutions in an arbirary time $t_{m}$, it is convenient for us to 
incorporate a factor $e^{t_{m} k^{m}}$ in $\Psi_{0}$ for each $t_{m}$, and consider series $\Psi_{0}(t ; k)$ of the form

$$
\Psi_{0}(t ; k)=\left(1+\sum_{s=1}^{\infty} \xi_{s}(t) k^{-s}\right) \exp \left(\sum_{i=1}^{\infty} t_{i} k^{i}\right) .
$$

As usual, all the times $t_{i}$ except for a finite number have been set to 0 . Then the operators $L_{m}$ are uniquely defined by the requirement that

$$
\left(\partial_{m}-L_{m}\right) \Psi_{0}(t ; k)=O\left(k^{-1}\right) \exp \left(\sum_{i=1}^{\infty} t_{i} k^{i}\right)
$$

(this is equivalent to the earlier requirement that $\left(L_{m}-k^{m}\right) \Psi_{0}=O\left(k^{-1}\right) \Psi_{0}$ in the case of $\xi_{s}(t)$ independent of $\left.t_{m}\right)$. In particular, we have the following identity between formal power series

$$
\left[\partial_{n}-L_{n}, \partial_{m}-L_{m}\right] \Psi_{0}=O\left(k^{-1}\right) \exp \left(\sum_{i=1}^{\infty} t_{i} k^{i}\right) .
$$

This identity assumes its full value when the formal series $\Psi_{0}(t ; k)$ is a genuine convergent function of $k$ and has an analytic continuation as a meromorphic function with $g$ poles on a Riemann surface of genus $g$. In this case, the equation (3.2) with zero right hand side becomes exact. The null space of $\left[\partial_{n}-L_{n}, \partial_{m}-L_{m}\right]$ is parametrized then by $k$ and is infinite-dimensional. Since $\left[\partial_{n}-L_{n}, \partial_{m}-L_{m}\right]$ is an ordinary differential operator, it must vanish. Thus a convergent $\Psi_{0}(t ; k)$ gives rise to a solution of the zero curvature equation $\left[\partial_{n}-L_{n}, \partial_{m}-L_{m}\right]=0$. The algebraic-geometric theory of solitons provides precisely the geometric data which leads to convergent Bloch functions. These functions are now known as Baker-Akhiezer functions.

\section{A. Geometric Data and Baker-Akhiezer Functions}

In a Baker-Akhiezer function, the spectral parameter $k$ is interpreted as the inverse $k=z^{-1}$ of a local coordinate $z$ on a Riemann surface. Thus let a "geometric data" $(\Gamma, P, z)$ consist of a Riemann surface $\Gamma$ of some fixed genus $g$, a puncture $P$ on $\Gamma$, and a local coordinate $k^{-1}$ near $P$. Let $\gamma_{1}, \ldots, \gamma_{g}$ be $g$ points of $\Gamma$ in general position. Then for any $t=\left(t_{i}\right)_{i=1}^{\infty}$, only a finite number of which are non-zero, there exists a unique function $\Psi(t ; z)$ satisfying

(i) $\Psi$ is a meromorphic on $\Gamma \backslash P$, with at most simple poles at $\gamma_{1}, \ldots, \gamma_{g}$;

(ii) in a neighborhood of $P, \Psi$ can be expressed as a convergent series in $k$ of the form appearing on the right hand side of (3.1).

The exponential factor in (3.1) describes the essential singularity of $\Psi(t ; z)$ near $P$. Alternatively, we can view it as a transition function (on the overlap between $\Gamma \backslash P$ and a neighborhood of $P$ ) for a line bundle $\mathcal{L}(t)$ on $\Gamma$. The Baker-Akhiezer function $\Psi$ is then a section of $\mathcal{L}(t)$, meromorphic on the whole of $\Gamma$. 
The form of the essential singularity of $\Psi$ implies that $\Psi$ has as many zeroes as it has poles (equivalently, the line bundle $\mathcal{L}(t)$ has vanishing Chern class). Indeed, $d \Psi / \Psi=d\left(\sum_{i=1}^{\infty} t_{i} k^{-i}\right)+$ regular, and thus has no residue at $P$. From this, the uniqueness of the Baker-Akhiezer function follows, since the ratio $\Psi / \tilde{\Psi}$ of two Baker-Akhiezer functions would be a meromorphic function on the whole of $\Gamma$, with at most $g$ poles (corresponding to the zeroes of $\tilde{\Psi}$ ). By the Riemann-Roch Theorem, it must be constant. Finally, the existence of $\Psi$ can be deduced most readily from an explicit formula. Let $A_{1}, \ldots, A_{g} B_{1}, \ldots, B_{g}$ be a canonical homology basis for $\Gamma$

$$
A_{j} \cap A_{k}=B_{j} \cap B_{k}=0, A_{j} \cap B_{k}=\delta_{j k},
$$

and let

(a) $d \omega_{j}, \tau_{j k}$ be respectively the dual basis of holomorphic abelian differentials and the period matrix

$$
\oint_{A_{j}} d \omega_{k}=\delta_{j k}, \oint_{B_{j}} d \omega_{k}=\tau_{j k}
$$

(b) $\theta(z \mid \tau)$ the Riemann $\theta$-function;

(c) $d \Omega_{i}^{0}$ the Abelian differential of the second kind with unique pole of the form

$$
d \Omega_{i}^{0}=\left(1+O\left(k^{-i-1}\right)\right) d k^{i}
$$

normalized to have vanishing $A$-periods

$$
\oint_{A_{I}} d \Omega_{i}^{0}=0,1 \leq I \leq g
$$

(d) $P_{0}$ a fixed reference point, with which we can define the Abel map $A$ : $z \in \Gamma \rightarrow A(z) \in \mathbf{C}^{g}$ and Abelian integrals $\Omega_{i}^{0}$ by

$$
A_{j}(z)=\int_{P_{0}}^{z} d \omega_{j}, \Omega_{i}^{0}(z)=\int_{P_{0}}^{z} d \Omega_{i}^{0}
$$

Here the Abel map as well as the Abelian integrals are path dependent, and we need to keep track of the path, which is taken to be the same in both cases.

(e) $Z=K-\sum_{s=1}^{g} A\left(\gamma_{s}\right)$, where $K$ is the vector of Riemann constants (c.f. [48]).

Using the transformation laws for the $\theta$-function, it is then easily verified that the following expression is well defined, and must coincide with $\Psi(t ; z)$

$$
\Psi(t ; z)=\frac{\theta\left(A(z)+\frac{1}{2 \pi i} \sum_{i=1}^{\infty} t_{i} \oint_{B} d \Omega_{i}^{0}+Z \mid \tau\right) \theta(A(P)+Z)}{\theta(A(z)+Z) \theta\left(A(P)+\frac{1}{2 \pi i} \sum_{i=1}^{\infty} t_{i} \oint_{B} d \Omega_{i}^{0}+Z \mid \tau\right)} \exp \left(\sum_{i=1}^{\infty} t_{i} \Omega_{i}^{0}(z)\right) .
$$


In this formalism, the role of the quasi-momentum $p$ is now assumed by the Abelian integral $\Omega_{1}$ with unique pole at $P$ of the first order normalized to have pure imaginary periods on $\Gamma$. With the Baker-Akhiezer function assuming now the former role of formal Bloch functions, we can construct a hierarchy of operators $L_{n}$ as in (2.37). The requirement that $\left(\partial_{m}-L_{m}\right) \Psi(t ; z)=O(z) \Psi(t ; z)$ determines recursively the coefficients of $L_{m}$ as differential polynomials in the $\xi_{s}$. The crucial improvement over formal Bloch functions is that here, this requirement actually implies that

$$
\left(\partial_{n}-L_{n}\right) \Psi(t ; z)=0
$$

identically. In fact, $\left(\partial_{n}-L_{n}\right) \Psi(t ; z)$ satisfies all the conditions for a BakerAkhiezer function, except for the fact that the Taylor expansion of its coefficient in front of the essential singularity $\exp \left(\sum_{i=1}^{\infty} t_{i} k^{i}\right)$ starts with $k^{-1}$. If this function is not identically zero, it can be used to generate distinct Baker-Akhiezer functions from any given one, contradicting the uniqueness of Baker-Akhiezer functions. As noted earlier, (3.8) implies that $\left[\partial_{n}-L_{n}, \partial_{m}-L_{m}\right] \Psi(t ; z)=0$, and hence that the zero curvature equation $\left[\partial_{n}-L_{n}, \partial_{m}-L_{m}\right]=0$ holds. In summary, we have defined in this way a "geometric map' $\mathcal{G}$ which sends the geometric data $\left(\Gamma, P, z ; \gamma_{1}, \ldots, \gamma_{g}\right)$ to an infinite hierarchy of operators [33][34]

$$
\mathcal{G}:\left(\Gamma, P, z ; \gamma_{1}, \ldots, \gamma_{g}\right) \rightarrow\left(L_{n}\right)_{n=2}^{\infty}
$$

The expression (3.7) for $\Psi(t ; z)$ leads immediately to explicit solutions for a whole hierarchy of soliton equations. Let $t_{1}=x, t_{2}=y, t_{3}=t$, and $n=2$. We find then solutions $u(x, y, t)$ of the KP equation (2.4) expressed as [34]

$$
u(x, y, t)=2 \partial_{x}^{2} \log \theta\left(x \oint_{B} d \Omega_{1}^{0}+y \oint_{B} d \Omega_{2}^{0}+t \oint_{B} d \Omega_{3}^{0}+Z \mid \tau\right)+\text { const. }
$$

This formula is at the origin of a remarkable application of the theory of non-linear integrable models, namely to a solution of the famous RiemannSchottky problem. According to the Torelli theorem, the period matrix defines uniquely the algebraic curve. The Riemann-Schottky problem is to describe the symmetric matrices with positive imaginary part which are period matrices of algebraic curves. Novikov conjectured that the function $u(x, y, t)=$ $2 \partial_{x}^{2} \log \theta(U x+V y+W t \mid \tau)$ is a solution of the KP equation if and only if the matrix $\tau$ is the period matrix of an algebraic curve, and $U, V, W$ are the $B$ periods of the corresponding normalized meromorphic differentials with poles only at a fixed point of the curve. This conjecture was proved in [55].

\section{The dual Baker-Akhiezer function}

For later use, we also recall here the main properties of the dual Baker-Akhiezer function $\Psi^{+}(t ; z)$ which coincides with the formal dual series defined in Section II.D. To define $\Psi^{+}(t ; z)$, we note that, given $g$ points $\gamma_{1}, \ldots, \gamma_{g}$ in general position, the unique meromorphic differential $d \Omega=d\left(z^{-1}+\sum_{s=2}^{\infty} a_{s} z^{s}\right)$ with 
double pole at $P$ and zeroes at $\gamma_{1}, \ldots, \gamma_{g}$, must also have $g$ other zeroes, by the Riemann-Roch theorem. Let these additional zeroes be denoted by $\gamma_{1}^{+}, \ldots, \gamma_{g}^{+}$. Then the dual Baker-Akhiezer function $\Psi^{+}(t ; z)$ is the unique function $\Psi^{+}(t ; z)$ which is meromorphic everywhere except at $P$, has at most simple poles at $\gamma_{1}^{+}, \ldots, \gamma_{g}^{+}$, and admits the following expansion near $P$

$$
\Psi^{+}(t ; z)=\exp \left(-\sum_{n=1}^{\infty} t_{n} z^{-n}\right)\left(1+\sum_{s=1}^{\infty} \xi_{s}^{+}(t) z^{s}\right)
$$

To compare the dual Baker-Akhiezer function with the formal dual Bloch function $\Psi_{0}^{+}$of Section II.E, it suffices to observe that

$$
\operatorname{Res}_{P} \Psi^{+}(t ; z)\left(\partial_{x}^{m} \Psi(t ; z)\right) d \Omega=0,
$$

since the differential on the left hand side is meromorphic everywhere, and holomorphic away from $P$. Together with the normalization $\Psi^{+}(0 ; z)=1$, this implies that $\Psi^{+}$indeed coincides with the formal dual function $\Psi_{0}^{+}$. An exact formula for $\Psi^{+}(t ; z)$ can be obtained from (3.7) by changing signs for $t$ and by replacing the vector $Z$ by $Z^{+}$. From the definition of the dual set of zeroes $\gamma_{1}^{+}, \ldots, \gamma_{g}^{+}$, this vector satisfies the equation $Z+Z^{+}=2 P+K$, where $K$ is the canonical class. Recalling that the quasi-momentum $p$ was defined to be $p=\Omega_{1}$, we also obtain the following formula for the differential $d \Omega$ we introduced earlier

$$
d \Omega=\frac{d p}{\langle\Psi+\Psi\rangle}
$$

\section{The Multi-Puncture Case}

The above formalism extends easily to the case of $N$ punctures $P_{\alpha}$ (with one marked puncture $P_{1}$ ). The Baker-Akhiezer function $\Psi$ is required then to have the essential singularity

$$
\Psi(t ; z)=\left(\sum_{s=0}^{\infty} \xi_{s \alpha}(t) k_{\alpha}^{-s}\right) \exp \left(\sum_{i=1}^{\infty} t_{i \alpha} k_{\alpha}^{i}\right),
$$

where $k_{\alpha}^{-1}$ are local coordinates near each puncture $P_{\alpha}, t_{i \alpha}$ are given "times", only a finite number of which are non-zero, and the coefficient $\xi_{s 1}$ at $P_{1}$ is normalized to be 1 for $s=0$. We can introduce as before $d \Omega_{i \alpha}^{0}$ associated now to each puncture $P_{\alpha}$ and their Abelian integrals $\Omega_{i \alpha}^{0}$. Then the Baker-Akhiezer function $\Psi(t ; z)$ becomes

$$
\begin{array}{r}
\Psi(t ; z)=\frac{\theta\left(A(z)+\frac{1}{2 \pi i} \sum_{\alpha=1}^{N} \sum_{i=1}^{\infty} t_{i \alpha} \oint_{B} d \Omega_{i \alpha}^{0}+Z \mid \tau\right) \theta\left(A\left(P_{1}\right)+Z\right)}{\theta(A(z)+Z) \theta\left(A\left(P_{1}\right)+\frac{1}{2 \pi i} \sum_{\alpha=1}^{N} \sum_{i=1}^{\infty} t_{i \alpha} \oint_{B} d \Omega_{i \alpha}^{0}+Z \mid \tau\right)} \\
\times \exp \left(\sum_{\alpha=1}^{N} \sum_{i=1}^{\infty} t_{i \alpha} \Omega_{i \alpha}^{0}(z)\right)
\end{array}
$$


For each pair $(\alpha, n)$ there is now a unique operator $L_{\alpha n}$ of the form (2.36) so that $\left(\partial_{\alpha n}-L_{\alpha n}\right) \psi(t, z)=0$, with $\partial_{\alpha n}=\partial / \partial t_{\alpha n}$. The operators $L_{\alpha n}$ satisfy the compatibility condition $\left[\partial_{\alpha n}-L_{\alpha n}, \partial_{\beta m}-L_{\beta m}\right]=0$.

\section{Periodic Solutions}

In general, the finite-gap solutions of soliton equations obtained by the above construction are meromorphic, quasi-periodic functions in each of the variables $t_{\alpha i}$ (a quasi-periodic function of one variable is the restriction to a line of a periodic function of several variables). We would like to single out the geometric data which leads to periodic solutions. For this we need the following slightly different formula for the Baker-Akhiezer function.

Let $d \Omega_{i \alpha}$ be the unique differential with pole of the form (3.4) near $P_{\alpha}$, but normalized so that all its periods be purely imaginary, and define the function $\Phi\left(\zeta_{1}, \ldots, \zeta_{2 g} ; z\right)$ by the formula

$$
\Phi(\zeta ; z)=\frac{\theta\left(A(z)+\zeta_{k} e_{k}+\zeta_{k+g} \tau_{k}+Z \mid \tau\right) \theta\left(A\left(P_{1}\right)+Z\right)}{\theta(A(z)+Z) \theta\left(A\left(P_{1}\right)+\zeta_{k} e_{k}+\zeta_{k+g} \tau_{k}+Z \mid \tau\right)} \exp \left(2 \pi i \sum_{k=1}^{g} A_{k}(z) \zeta_{k+g}\right)
$$

where $e_{k}=(0, \ldots, 0,1,0, \ldots, 0)$ are the basis vectors in $\mathbf{C}^{g}$, and $\tau_{k}$ are the vectors with components $\tau_{j k}$. We observe that $\Phi$ is periodic of period 1 in each of the variables $\zeta_{1}, \ldots, \zeta_{2 g}$. Then the Baker-Akhiezer function can be expressed as

$$
\Psi(t ; z)=\Phi\left(\sum_{i \alpha} t_{i \alpha} U_{i \alpha} ; z\right) \exp \left(\sum_{i \alpha} t_{i \alpha} \Omega_{i \alpha}\right)
$$

where we have denoted by $U_{i \alpha}$ the real, $2 g$-vector of periods of $d \Omega_{i \alpha}$

$$
U_{i \alpha}^{k}=\frac{1}{2 \pi i} \oint_{A_{k}} d \Omega_{i \alpha}, \quad U_{i \alpha}^{k+g}=-\frac{1}{2 \pi i} \oint_{B_{k}} d \Omega_{i \alpha}
$$

In particular, for geometric data $\left\{\Gamma, P_{\alpha}, z_{\alpha}\right\}$ satisfying the condition

$$
\frac{1}{2 \pi i} \sum_{i \alpha} a_{i \alpha} U_{i \alpha} \in \mathbf{Z}
$$

the Baker-Akhiezer function is a Bloch function with respect to the variable $\tilde{t}$ if we set $t_{i \alpha}=a_{i \alpha} \tilde{t}$, with Bloch multiplier $w=\exp \left(\sum_{i \alpha} a_{i \alpha} \Omega_{i \alpha}(z)\right)$. The coefficients of the operators $L_{\alpha n}$ are then periodic functions of $\tilde{t}$. As an example, we consider the one-puncture case. If we express the data under the form $U_{1}^{k}=$ $2 \pi m_{k} / l_{1}, U_{2}^{k}=2 \pi n_{k} / l_{2}$, with $m_{k}, n_{k} \in \mathbf{Z}$, then the corresponding solution of the KP hierarchy is periodic in the variables $x=t_{1}, y=t_{2}$, with periods $l_{1}$ and $l_{2}$ respectively. 


\section{Real and Smooth Solutions}

There are two types of conditions which guarantee that the solutions obtained by the above geometric construction are real and smooth for real values of $t_{i \alpha}$. We present them in the case of the KP hierarchy.

Assume that the geometric data defining the Baker-Akhiezer function is real, in the sense that

(a) the algebraic curve $\Gamma$ admits an anti-holomorphic involution $\iota: \Gamma \rightarrow \Gamma$;

(b) the puncture $P_{1}$ is a fixed point of $\iota$;

(c) the local coordinate $k^{-1}$ in a neighborhood of $P_{1}$ satisfies the condition $k(\iota(z))=\overline{k(z)}$;

(d) the divisor $\left(\gamma_{1}, \ldots, \gamma_{g}\right)$ is invariant under $\iota$, i.e., $\iota\left(\gamma_{s}\right)=\gamma_{\sigma(s)}$, where $\sigma$ is a permutation.

Then the Baker-Akhiezer function satisfies the reality condition

$$
\Psi(t ; \iota(z))=\overline{\Psi(t ; z)} .
$$

This is an immediate consequence of the uniqueness of the Baker-Akhiezer function and the fact that both sides of the equation have the same analytic properties. In particular, the coefficients of $L_{n}$ and the corresponding solutions of the KP hierarchy are real.

In order to have real and smooth solutions, it is necessary to restrict further the geometric data. In general, the set of fixed points of any anti-holomorphic involution on a smooth Riemann surface is a union of disjoint cycles. The number of these cycles is less or equal to $g+1$. The algebraic curves which admit an anti-involution with exactly $g+1$ fixed cycles are called $M$-curves. We claim that the coefficients of $L_{n}$ are real and smooth functions of all variables $t_{i}$ when $\Gamma$ is an M-curve with fixed cycles $A_{0}, A_{1}, \ldots, A_{g}$, and $P \in A_{0}, \gamma_{s} \in A_{s}$, $s=1, \ldots, g$. To see this, we note that, from the explicit expression for the Baker-Akhiezer function, the coefficients of $L_{n}$ have poles at some value of $t_{i}$ if and only if

$$
\theta\left(A\left(P_{1}\right)+\sum_{i} U_{i} t_{i}+Z\right)=0 .
$$

The monodromy properties of the $\theta$-function imply that the zeros of the function $\theta\left(A(z)+\sum_{i} U_{i} t_{i}+Z\right)$ are well-defined on $\Gamma$, even though the function itself is multi-valued. The number of these zeroes is $g$. They coincide with the zeroes of $\Psi(t, z)$. In view of (3.17), the Baker-Akhiezer function is real on the cycles $A_{s}$. On each of the cycles $A_{1}, \ldots, A_{g}$, there is one pole of $\Psi$. There must then be at least one zero on the same cycle. Hence all zeroes of $\theta\left(A(z)+\sum_{i} U_{i} t_{i}+Z\right)$ are located on cycles $A_{s}$. Since $P_{1} \in A_{0}$, the equation (3.18) cannot be fulfilled for real values of $t_{i}$.

We observe that the real and smooth solutions of the KP hierarchy corresponding to M-curves with a fixed puncture, are parametrized by the points of 
a real $g$-dimensional torus which is the product of the $g$ cycles $A_{s}$. If we choose these cycles (as our notation suggests) as half of a canonical basis of cycles, then this torus is the real part of the Jacobian.

In the theory of real and smooth solutions the equation (2.4) is called the KP-2 equation. The other, so-called, KP-1 equation is the other real form of the same equation. It can be obtained from (2.4) by changing $y$ to $i y$. Thus the KP-1 equation is given explicitly by

$$
-\frac{3}{4} u_{y y}=\left(u_{t}-\frac{3}{2} u u_{x}-\frac{1}{4} u_{x x x}\right)_{x} .
$$

As complex equations (2.4) and (3.19) are equivalent. But the conditions which single out real and smooth solutions are different. These conditions for the KP-1 equation may be found in [37]. Briefly they are :

Assume that the geometric data defining the Baker-Akhiezer function is real, in the sense that

(a) the algebraic curve $\Gamma$ admits an anti-holomorphic involution $\iota: \Gamma \rightarrow \Gamma$;

(b) the puncture $P_{1}$ is a fixed point of $\iota$;

(c) the local coordinate $k^{-1}$ in a neighborhood of $P_{1}$ satisfies the condition $k(\iota(z))=-\overline{k(z)}$;

(d) the divisor $\left(\gamma_{1}, \ldots, \gamma_{g}\right)$ under $\iota$ becomes the dual divisor $\gamma_{1}^{+}, \ldots, \gamma_{g}^{+}$i.e., $\iota\left(\gamma_{s}\right)=\gamma_{\sigma(s)}^{+}$, where $\sigma$ is a permutation.

Then the Baker-Akhiezer function satisfies the reality condition

$$
\Psi^{+}\left(t^{\prime} ; \iota(z)\right)=\overline{\Psi\left(t^{\prime} ; z\right)}
$$

where the new variables $t^{\prime}=\left(t_{1}^{\prime}, \ldots\right)$ are equal to $t_{2 m+1}^{\prime}=t_{2 m+1}, t_{2 m}=i t_{2 m}$. As before, this is an immediate consequence of the uniqueness of the BakerAkhiezer function and the fact that both sides of the equation have the same analytic properties. In particular, the coefficients of $L_{n}$ and the corresponding solutions of the KP-1 hierarchy are real for real values of $t^{\prime}$.

The further restriction of geometric data corresponding to real and smooth solutions of the KP-1 hierarchy is as follows. The fixed cycles $a_{1}, \ldots, a_{l}$ of $\iota$ should divide $\Gamma$ into two disconnected domaines $\Gamma^{ \pm}$. The complex domain $\Gamma^{+}$ defines the orientation on the cycles considered as its boundary. The differential $d \Omega$ of (3.11) should be positive on $a_{s}$ with respect to this orientation.

\section{B. Moduli Spaces of Surfaces and Abelian Integrals}

The space $\left\{\Gamma, P, z, \gamma_{1}, \ldots, \gamma_{g}\right\}$ provides geometric data for solutions of a complete hierarchy of soliton equations, and is infinite-dimensional. In the remaining part of this paper, we concentrate rather on a single equation of zero curvature form $\left[\partial_{y}-L, \partial_{t}-A\right]=0$. The geometric data associated with the 
pair $(L, A)$ corresponds to the Jacobian bundle over a finite-dimensional moduli space $\mathcal{M}_{g}(n, m)$ of Riemann surfaces with a pair of Abelian integrals $(E, Q)$ with poles of order $n$ and $m$ respectively at the puncture $P$. The associated operators $(L, A)$ are then operators of order $n$ and $m$, and are obtained by the basic construction (3.9), after imbedding $\mathcal{M}_{g}(n, m)$ in the space $(\Gamma, P, z)$ of geometric data. Alternatively, we may choose to represent the equation $\left[\partial_{y}-L, \partial_{t}-A\right]=0$ as a dynamical system on a space of operators $L$, with $t$ as time variable. In this case, a finite-dimensional and geometric space of operators $L$ is obtained by the same construction as just outlined, starting instead from the Jacobian bundle over the moduli space $\mathcal{M}_{g}(n)$ of Riemann surfaces $\Gamma$ with just one Abelian integral with pole of order $n$ at the puncture.

More precisely, given $(\Gamma, E)$, a geometric data $(\Gamma, P, z)$ is obtained by setting the local coordinate $z \equiv K^{-1}$ near the puncture $P$ to be

$$
E=z^{-n}+R^{E} \log z
$$

where $n \geq 1$ and $R^{E}$ are respectively the order of the pole of $E$ and its residue at $P$. When $n=0$, we set instead

$$
E=R^{E} \log z
$$

This gives immediately a map

$$
\begin{aligned}
(\Gamma, E) & \rightarrow(\Gamma, P, z), \\
\left(\Gamma, E, \gamma_{1}, \ldots, \gamma_{g}\right) & \rightarrow\left(\Gamma, P, z, \gamma_{1}, \ldots, \gamma_{g}\right) \rightarrow L,
\end{aligned}
$$

where the operator $L$ is characterized by the condition $\left(\partial_{y}-L\right) \Psi=0$, with $\Psi(x, y ; k)$ the Baker-Akhiezer function having the essential singularity $\exp (k x+$ $\left.k^{n} y\right), k=z^{-1}$. In presence of a second Abelian integral $Q$, we can select a second time $t$, by writing the singular part $Q_{+}(k)$ of $Q$ as a polynomial in $k$ and setting

$$
\begin{aligned}
Q_{+}(k) & =a_{1} k+\ldots+a_{m} k^{m}, \\
t_{i} & =a_{i} t, 1 \leq i \leq m .
\end{aligned}
$$

This means that we consider the Baker-Akhiezer function $\Psi(x, y, t ; k)$ with the essential singularity $\exp \left(k x+k^{n} y+Q_{+}(k) t\right)$, and construct the operators $L$ and $A$ by requiring that $\left(\partial_{y}-L\right) \Psi=\left(\partial_{t}-A\right) \Psi=0$. The pair $(L, A)$ provides then a solution of the zero-curvature equation. By rescaling $t$, we can assume that $A$ is monic. Altogether, we have restricted the geometric map $\mathcal{G}$ of (3.9) to a map on finite-dimensional spaces, which we still denote by $\mathcal{G}$

$$
\begin{aligned}
\mathcal{G}:\left(\Gamma, E ; \gamma_{1}, \ldots \gamma_{g}\right) & \rightarrow(\Gamma, P, z) \rightarrow(L), \\
\mathcal{G}:\left(\Gamma, E, Q ; \gamma_{1}, \ldots \gamma_{g}\right) & \rightarrow(\Gamma, P, z, t) \rightarrow(L, A) .
\end{aligned}
$$

Here we have indicated explicitly the choice of time in the geometric data. The proper interpretation of the full geometric data $\left(\Gamma, E, Q ; \gamma_{1}, \ldots \gamma_{g}\right)$ is as a 
point in the bundle $\mathcal{N}_{g}^{g}(n, m)$ over $\mathcal{M}_{g}(n, m)$, whose fiber is the $g$-th symmetric power $S^{g}(\Gamma)$ of the curve. The $g$-th symmetric power can be identified with the Jacobian of $\Gamma$ via the Abel map

$$
\left(\gamma_{1}, \ldots, \gamma_{g}\right) \rightarrow \phi_{j}=\sum_{i=1}^{g} \int_{P_{1}}^{\gamma_{i}} d \omega_{j}
$$

More generally, we can construct the bundles $\mathcal{N}_{g}^{k}(n, m)$ and $\mathcal{N}_{g}^{k}(n)$ with fiber $S^{k}(\Gamma)$ over the bases $\mathcal{M}_{g}(n, m)$ and $\mathcal{M}_{g}(n)$ respectively

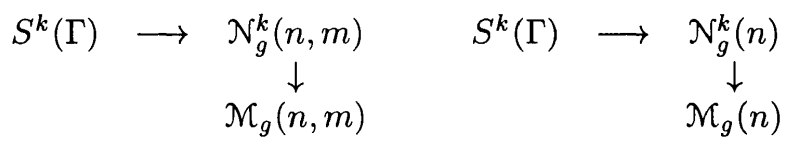

Thus the bundles $\mathcal{N}_{g}^{k=1}(n, m) \equiv \mathcal{N}_{g}(n, m)$, and $\mathcal{N}_{g}^{k=1}(n) \equiv \mathcal{N}_{g}(n)$ are the analogues in the our context of the universal curve. Returning to soliton equations, the geometric map $\mathcal{G}$ of (3.25) can now be succinctly described as a map from the fibrations $\mathcal{N}_{g}^{g}(n)$ and $\mathcal{N}_{g}^{g}(n, m)$ into the spaces respectively of operators $L$ and pairs $(L, A)$ of operators

$$
\begin{gathered}
\mathcal{G}: \mathcal{N}_{g}^{g}(n) \rightarrow(L), \\
\mathcal{G}: \mathcal{N}_{g}^{g}(n, m) \rightarrow(L, A) .
\end{gathered}
$$

We emphasize that, although the operators in its image are not all periodic operators, the ones arising later upon restriction of $\mathcal{G}$ to suitable subvarieties of $\mathcal{N}_{g}^{g}(n, m)$ and $\mathcal{N}_{g}^{g}(n)$ with integral periods (c.f. Section III.C) will be.

We conclude this section by observing that, in the preceding construction, $L$ and $A$ depend only the singular part of $Q$, and hence are unaffected if $d Q$ is shifted by a holomorphic differential. As we shall see below, the appropriate normalization in soliton theory is the real normalization by which we require that

$$
\operatorname{Re} \oint_{C} d Q=0
$$

In the study of $\mathrm{N}=2$ supersymmetric gauge theories, holomorphicity is a prime consideration, and we shall rather adopt in this context the complex normalization

$$
\oint_{A_{j}} d Q=0,1 \leq j \leq g
$$

We note that each normalization provides an imbedding of $\mathcal{M}_{g}(n)$ into $\mathcal{M}_{g}(n, 1)$, by making the choice $Q_{+}(K)=K$, with periods satisfying either (3.29) or (3.30), so that the operator $A$ is just $A=\partial_{x}$ in either case. The image of $\mathcal{M}_{g}(n)$ in $\mathcal{M}_{g}(n, 1)$ does depend on the normalization, however. 


\section{Geometric Symplectic Structures}

We begin by discussing the basic local geometry of the moduli spaces $\mathcal{M}_{g}(n)$ and $\mathcal{M}_{g}(n, m)$. They are complex manifolds with only orbifold singularities, of dimensions

$$
\begin{aligned}
\operatorname{dim} \mathcal{M}_{g}(n) & =4 g-3+2 N+\sum_{\alpha=1}^{N} n_{\alpha}, \\
\operatorname{dim} \mathcal{M}_{g}(n, m) & =5 g-3+3 N+\sum_{\alpha=1}^{N}\left(n_{\alpha}+m_{\alpha}\right) .
\end{aligned}
$$

Indeed, the number of degrees of freedom of an Abelian integral $E$ with poles of order $n=\left(n_{\alpha}\right)$ is $1+\sum_{\alpha=1}^{N}\left(n_{\alpha}+1\right)-1+g=N+g+\sum_{\alpha=1}^{N} n_{\alpha}$, where the first 1 corresponds to the additive constant, and the remaining integer on the left is the dimension of meromorphic differentials with poles of order $\leq n_{\alpha}+1$ at each $P_{\alpha}$. For $g>1$, the dimension of the moduli space of Riemann surfaces with $N$ punctures is $3 g-3+N$, which leads immediately to (3.31). For $g \leq 1$, it is easily verified that the same formula (3.31) holds, although the counting has to incorporate holomorphic vector fields and is slightly different in intermediate stages.

We can introduce explicit local coordinates on $\mathcal{M}_{g}(n, m)$. To obtain welldefined branches of Abelian integrals, we cut apart the Riemann surface $\Gamma$ along a canonical homology basis $A_{i}, B_{j}, i, j=1, \ldots, g$, and along cuts from $P_{1}$ to $P_{\alpha}$ for each $2 \leq \alpha \leq N$. Locally on $\mathcal{M}_{g}(n, m)$, this construction can be carried out continuously, with paths homotopic by deformations not crossing any of the poles. Denote the resulting surface by $\Gamma_{\text {cut }}$. On $\Gamma_{\text {cut }}$, the Abelian integrals $E$ and $Q$ become single-valued holomorphic functions, and we can introduce the one-form $d \lambda$ by

$$
d \lambda=Q d E \text {. }
$$

We observe that $d \lambda$ has a singularity of order $n_{\alpha}+m_{\alpha}+1$ at each puncture $P_{\alpha}$. Now the Abelian integral $E$ defines a coordinate system $z_{\alpha}$ near each $P_{\alpha}$ by

$$
E=z_{\alpha}^{-n_{\alpha}}+R_{\alpha}^{E} \log z_{\alpha}
$$

when $n_{\alpha}$ is strictly positive. When $n_{\alpha}=0$, we write instead

$$
E=R_{\alpha}^{E} \log z_{\alpha}
$$

The coordinate $z_{\alpha}$ can be used to fix the additive normalization of the Abelian integral $\lambda$, and to describe its Laurent expansion near each puncture. Thus we fix the additive constant in $\lambda$ by demanding that its expansion in $z_{1}$ near $P_{1}$ have no constant term. The parameters $T_{\alpha, i}, 1 \leq i \leq n_{\alpha}+m_{\alpha}, R_{\alpha}^{\lambda}, 2 \leq \alpha \leq N$, can then be defined by

$$
\begin{aligned}
T_{\alpha, i} & =-\frac{1}{i} \operatorname{Res}_{P_{\alpha}}\left(z_{\alpha}^{i} d \lambda\right), 1 \leq \alpha \leq N, 1 \leq i \leq n_{\alpha}+m_{\alpha} \\
R_{\alpha}^{\lambda} & =\operatorname{Res}_{P_{\alpha}}(d \lambda) .
\end{aligned}
$$


The parameters $T_{\alpha, i}(1 \leq \alpha \leq N)$ and $R_{\alpha}^{\lambda}(2 \leq \alpha \leq N)$ account for $\sum_{\alpha=1}^{N}\left(n_{\alpha}+\right.$ $\left.m_{a}\right)+N-1$ parameters. The remaining parameters needed to parametrize $\mathcal{M}_{g}(n, m)$ consist of the $2 N-2$ residues of $d E$ and $d Q$

$$
R_{\alpha}^{E}=\operatorname{Res}_{P_{\alpha}} d E, R_{\alpha}^{Q}=\operatorname{Res}_{P_{\alpha}} d Q, \alpha=2, \ldots, N,
$$

and the following $5 g$ parameters which account for the presence of non-trivial topology

$$
\begin{aligned}
\tau_{A_{i}, E} & =\oint_{A_{i}} d E, \tau_{B_{i}, E}=\oint_{B_{i}} d E \\
\tau_{A_{i}, Q} & =\oint_{A_{i}} d Q, \tau_{B_{i}, Q}=\oint_{B_{i}} d Q \\
a_{i} & =\oint_{A_{i}} Q d E, i=1, \ldots, g .
\end{aligned}
$$

Theorem 13. Let $\mathcal{D}$ be the open set in $\mathcal{M}_{g}(m, n)$ where the zero divisors of $d E$ and $d Q$, namely the sets $\{z ; d E(z)=0\}$ and $\{z ; d Q(z)=0\}$, do not intersect. Then

(a) Near each point in $\mathcal{D}$, the $5 g-3+3 N+\sum_{\alpha=1}^{N}\left(n_{\alpha}+m_{\alpha}\right)$ parameters $R_{\alpha}^{E}$, $R_{\alpha}^{Q}, R_{\alpha}^{\lambda}, T_{\alpha, k}, \tau_{A_{i}, E}, \tau_{B_{i}, E}, \tau_{A_{i}, Q}, \tau_{B_{i}, Q}, a_{i}$ have linearly independent differentials, and thus define a local holomorphic coordinate system for $\mathcal{M}_{g}(n, m)$;

(b) The joint level sets of the set of all parameters except $a_{i}$ define a smooth $g$-dimensional foliation of $\mathcal{D}$, independent of the choices we made to define the coordinates themselves.

Theorem 10 is proved in [39]. Since $\mathcal{M}_{g}(n)$ can be imbedded in $\mathcal{M}_{g}(n, m)$ by choosing $Q$ to have Laurent expansion $Q_{+}(K)=K$ and fixing its $A_{k}$ periods, Theorem 1 also provides local coordinates for $\mathcal{M}_{g}(n)$. Specifically, the coordinates of $\mathcal{M}_{g}(n)$ which arise this way are

- $T_{\alpha, i}$ with $1 \leq i \leq n_{\alpha}$ for $\alpha \geq 2, T_{1, i}$ with $1 \leq i \leq n_{1}-1$ (since the normalization $Q=K^{-1}+O(\bar{K})$ fixes the coefficients of the two leading terms $T_{1, n_{1}+1}$ and $T_{1, n_{1}}$ in the singularity expansion of $\lambda$ near $P_{1}$ to be $T_{1, n_{1}+1}=\frac{n_{1}}{n_{1}+1}, T_{1, n_{1}}=0$ );

- the $2 N-2$ residues of $d E$ and $d \lambda$ at $P_{\alpha}$ for $\alpha \geq 2$;

- the $B_{k}$ periods of $d Q$, the $A_{k}$ and $B_{k}$ periods of $d E$, and the $A_{k}$ periods of $d \lambda$,

for a total of $4 g-3+2 N+\sum_{\alpha=1}^{N} n_{\alpha}$.

The intrinsic foliation obtained in Theorem 10 is central to our considerations, and we shall refer to it as the canonical foliation. Our goal is to construct now a symplectic form $\omega$ on the complex $2 g$-dimensional space obtained 
by restricting the fibration $\mathcal{N}_{g}^{g}(n, m)$ to a $g$-dimensional leaf $\mathcal{M}$ of the canonical foliation of $\mathcal{M}_{g}(n, m)$. For this, we need to extend the differentials $d E$ and $d Q$ to one-forms on the whole fibration, and distinguish between the two ways this can be done.

One way is to consider the Abelian integrals $E$ and $Q$ as multi-valued functions on the fibration. Despite their multivaluedness, their differentials along any leaf of the canonical fibration are well-defined. In fact, $E$ and $Q$ are welldefined in a small neighborhood of the puncture $P_{1}$. The ambiguities in their values anywhere on each Riemann surface consist only of integer combinations of their residues or periods along closed cycles. Thus they are constant along any leaf of the canonical foliation, and disappear upon differentiation. The differentials along the fibrations obtained this way will be denoted by $\delta E$ and $\delta Q$. Restricted to vectors tangent to the fiber, they reduce of course to the differentials $d E$ and $d Q$.

The other way is to trivialize the fibration by using an Abelian integral, say $E$, as local coordinate in $\Gamma$ (c.f. (3.21) and (3.22)). Equivalently, we note that at any point of $\mathcal{N}$, the varieties $E=$ constant are intrinsic and transversal to the fiber. Thus any one-form $d Q$ on the fiber can be extended to a one-form on the total space of the fibration, by making it zero on vectors tangent to these varieties. We still denote this one-form by $d Q$. In the case of the Abelian integral $E$, we have $\delta E=d E$, but this is not true in general. To compare $d Q$ with $\delta Q$, let $a_{1}, \ldots, a_{g}$ be local coordinates for the leaf. Then $a_{1}, \ldots, a_{g}, E$ are local coordinates for the whole fibration, $d a_{1}, \ldots, d a_{g}, d E$ are a basis of one-forms, and $d Q=\frac{d Q}{d E} d E$. On the other hand,

$$
\delta Q=d Q+\sum_{i=1}^{g} \frac{\partial Q}{\partial a_{i}} d a_{i} \equiv d Q+\delta^{E} Q .
$$

Similarly, the full differential $\delta(Q d E)$ on the total space of the fibration is welldefined, despite the multivaluedness of $Q$. The partial derivatives $\partial_{a_{i}}(Q d E)$ along the base are all holomorphic, since both the singularities and the ambiguities in the differential are constant, and disappear upon differentiation. Recalling that $d \omega_{j}$ denotes the basis of holomorphic differentials dual to the homology basis $A_{k}, B_{k}$, we can then write

$$
\frac{\partial}{\partial a_{i}}(Q d E)=d \omega_{i}
$$

By extending this construction to the fibration of Jacobians over a leaf $\mathcal{M}$ of the canonical foliation, we obtain the desired geometric symplectic form. Furthermore, this symplectic form coincides with the symplectic form constructed in Section II.E, upon imbedding the fibration of Jacobians in the space of soliton solutions of the equation $\left[\partial_{y}-L, \partial_{t}-A\right]=0$ by the geometric map $\mathcal{G}$ of $(3.28)$ [39]:

Theorem 14. (a) The following two-form on the fibration $\mathcal{N}^{g}(n, m)$ restricted 
to a leaf $\mathcal{M}$ of the canonical foliation of $\mathcal{M}_{g}(n, m)$

$$
\omega_{\mathcal{M}}=\delta\left(\sum_{i=1}^{g} Q\left(\gamma_{i}\right) d E\left(\gamma_{i}\right)\right)=\sum_{i=1}^{g} \delta Q\left(\gamma_{i}\right) \wedge d E\left(\gamma_{i}\right)=\sum_{i=1}^{g} d a_{i} \wedge d \omega_{i}
$$

defines a symplectic form; (b) Under the geometric correspondence $\mathcal{G}$, we have

$$
\omega_{\mathcal{M C}}=\mathcal{G}^{*}\left(\omega_{m}\right)
$$

where $\omega_{m}$ is the symplectic form constructed in Theorem 7.

It is instructive enough to present a proof of Theorem 11 for the basic symplectic structure defined by (2.62) on a space of linear operators $L$ with scalar coefficients. In this case $Q=p$.

Let $\Psi, \Psi^{+}$be the Baker-Akhiezer and the dual Baker-Akhiezer functions corresponding to the algebraic-geometric data (3.25) (c.f. Sections III.A and III.B). Then

$$
\mathcal{G}^{*}(\omega)=\operatorname{Res}_{P}\left\langle\Psi^{*} \delta L \wedge \delta \Psi\right\rangle d p, \Psi^{*}=\Psi^{+}\left(\left\langle\Psi^{+} \Psi\right\rangle\right)^{-1} .
$$

The differential

$$
d \Omega^{*}=\left\langle\Psi^{*} \delta L \wedge \delta \Psi\right\rangle d p
$$

is meromorphic. Thus its residue at $P$ is the opposite of the sum of its other residues. The residues of $d \Omega^{*}$ at the poles $\gamma_{s}$ of $\Psi$ are given by

$$
\operatorname{Res}_{\gamma_{s}} d \Omega^{*}=\left\langle\Psi^{*} \delta L \Psi\right\rangle \wedge \delta p\left(\gamma_{s}\right)=\delta E\left(\gamma_{s}\right) \wedge \delta p\left(\gamma_{s}\right)
$$

In general, poles of $d \Omega^{*}$ may also arise from the zeroes $q_{i}$ of $d p, d p\left(q_{i}\right)=0$, which are singular points of the connection on $\mathcal{N}$ defined by $d p$. However,

$$
\operatorname{Res}_{q_{i}} d \Omega^{*}=\operatorname{Res}_{q_{i}}\left\langle\Psi^{*} \delta L d \Psi\right\rangle \wedge \delta p\left(q_{i}\right)=0,
$$

in view of the relations $\Psi^{*} \delta L=\delta \Psi^{*}\left(\partial_{y}-L\right)$ and $\left(\partial_{y}-L\right) d \Psi=0$. Theorem 11 is proved.

Remark. In general, when the Lax operator $L$ explicitly depends on a spectral parameter (as in the example of the Calogero-Moser system below), a special gauge should be chosen in order for $d \Omega^{*}$ to be regular at the branching points of the connection.

\section{Generalizations and Extensions}

The above set-up can be easily adapted to a variety of important equations.

For the 2D Toda lattice, the differential operator $\partial_{x}$ is replaced by a difference operator. Thus for the geometric data, we consider the moduli space of Riemann surfaces $\Gamma$ with two punctures $P_{ \pm}$, and Abelian differentials $E, Q$ which are real-normalized, with $E$ having a pole of order 1 at $P_{+}$, while $d Q$ has simple poles at $P_{ \pm}$. This is a leaf in the foliation of $\mathcal{M}_{g}(1,0 ; 0,0)$. The discrete 
analogue of the Baker-Akhiezer function $\Psi(z ; t)$ is now given by a sequence $\Psi_{n}\left(t_{+}, t_{-} ; z\right)$ of Baker-Akhiezer functions characterized by

$$
\Psi_{n}\left(t_{+}, t_{-} ; z\right)=z_{ \pm}^{ \pm n}\left(\sum_{s=0}^{\infty} \xi_{s}(n, t) z_{ \pm}^{s}\right) \exp \left(t_{+} z_{+}^{-1}+t_{-} z_{-}^{-1}\right)
$$

As in Section II.I, we define the operators $L$ and $A$ as difference operators acting on sequences $\psi_{n}$ which satisfy $\left(\partial_{t_{+}}-L\right) \Psi=\left(\partial_{t_{-}}-A\right) \Psi=0$. Their coefficients $c_{n}, v_{n}, \phi_{n}$ are now functions of the geometric data. In analogy with (3.43), we have then

$$
\begin{aligned}
\omega_{\mathcal{M}}=\mathcal{G}^{*}\left(\omega_{\mathcal{M}}\right) & =-\sum_{\alpha= \pm} \operatorname{Res}_{P_{ \pm}}\left\langle\Psi_{n}^{*} \delta L \wedge \delta \Psi_{n}\right\rangle d p=\sum_{n}\left\langle\delta \phi_{n} \wedge \delta v_{n}\right\rangle \\
d p & =\mp \frac{d z}{z}, z \rightarrow P_{ \pm} .
\end{aligned}
$$

Note that the difference between this formula and the one in Section II.J is due to the choice $t_{+}$instead of $t_{+}+t_{-}$as the second variable.

In the case of $N \times N$ matrix equations $\left[\partial_{y}-L, \partial_{t}-A\right]=0$, we take the geometric data to consist of surfaces $\Gamma$ with $N$ punctures $P_{\alpha}$, and $E, Q$ to be real-normalized Abelian integrals with poles of order $n$ and $m$ at $P_{\alpha}$. Let $a_{\alpha}$ be given coefficients, with $a_{1}=1$. The leaf $\mathcal{M}$ corresponding to the above specifications for $E, Q$, combines with the space of parameters $a_{\alpha}$ to a product space $\mathcal{M} \times \mathbf{C}^{N-1}$ of dimension $g+N-1$. On the fibration $\mathcal{N}^{g+N-1}$ above the product $\mathcal{M} \times \mathbf{C}^{N-1}$, we can construct the symplectic form

$$
\omega_{\mathcal{M}}=\sum_{s=1}^{g+N-1} Q\left(\gamma_{s}\right) d E\left(\gamma_{s}\right)
$$

Now corresponding to the preceding geometric data are local coordinates $z_{\alpha}$ near each puncture $P_{\alpha}$ given in analogy with (3.21) and (3.22) by $E=a_{\alpha} z_{\alpha}^{-n}$, polynomials $Q_{\alpha,+}\left(z_{\alpha}^{-1}\right)$ which are the singular parts of $Q$ near $P_{\alpha}$, and thus a vector Baker-Akhiezer function $\Psi(z ; x, y, t)=\left(\Psi_{\alpha}(z ; x, y, t)\right)_{\alpha=1}^{N}$ with the following essential singularity near $P_{\beta}$

$$
\Psi_{\alpha}(z ; x, y, t)=\left(\delta_{\alpha \beta}+\sum_{s=1}^{\infty} \xi_{s}^{\alpha \beta}(x, y, t) z_{\beta}^{s}\right) \exp \left(z_{\beta}^{-1} x+a_{\beta} z_{\beta}^{-n} y+Q_{\beta,+}\left(z_{\beta}^{-1}\right) t\right)
$$

As in Section II.I, there exist then unique matrix operators $L, A$ so that $\left(\partial_{y}-\right.$ $L) \Psi=\left(\partial_{t}-A\right) \Psi=0$. They have expressions of the form in (2.93) and subsequent equations. We observe that the case where $Q$ has only simple poles $\left(m_{i}=1, Q_{\beta,+}=z_{\beta}^{-1}\right)$ is the matrix generalization of the scalar case considered earlier, where $Q$ still has the interpretation of a quasi-momentum.

On the space of such operators, we had defined in Theorem 8 a symplectic form $\omega$. Here again, the geometric and the formal symplectic forms (3.46) and 
(2.96) correspond to one another under the basic geometric correspondence $\mathcal{G}$. More precisely,

$$
\omega_{\mathcal{M}}=\sum_{\alpha=1}^{N} \operatorname{Res}_{P_{\alpha}}\left\langle\Psi^{*}\left(A_{m}^{(1)} \delta L-L^{(1)} \delta A_{m}\right) \wedge \delta \Psi\right\rangle d p=\mathcal{G}^{*}\left(\omega_{m}\right),
$$

where

$$
\omega_{m}=\operatorname{Res}_{\infty} \operatorname{Tr}\left\langle\Psi_{0}^{*}\left(A_{m}^{(1)} \delta L-L^{(1)} \delta A_{m}\right) \wedge \delta \Psi_{0}\right\rangle d k .
$$

In the case $m_{i}=1$, we have $A=\partial_{x}$, and this relation reduces to

$$
\omega_{\mathcal{X}}=-\sum_{\alpha=1}^{N} \operatorname{Res}_{P_{\alpha}}\left\langle\Psi^{*} \delta L \wedge \delta \Psi\right\rangle d p=\mathcal{G}^{*}(\omega) .
$$

with $\omega$ given by (2.96).

Similarly, our formalism can easily identify the action coordinates for the elliptic Calogero-Moser system, an issue which had been resolved only relatively recently [29]. We recall that the elliptic Calogero-Moser system is a system of $N$ identical particles on a line, interacting with each other via the potential $V(x)=\wp(x)$

$$
\ddot{x}_{i}=4 \sum_{j \neq i} \wp^{\prime}\left(x_{i}-x_{j}\right), \quad \wp(x)=\frac{d \wp(x)}{d x} .
$$

Here $\wp(x)=\wp\left(x \mid \omega, \omega^{\prime}\right)$ is the Weierstrass elliptic function with periods $2 \omega, 2 \omega^{\prime}$, and $\omega, \omega^{\prime}$ are fixed parameters. The complete solution of the elliptic CalogeroMoser system was constructed by geometric methods in [35]. There an explicit Lax pair $(L, M)$ was found, depending on a spectral parameter $z$ varying on the torus $\mathbf{C} /\left(2 \omega \mathbf{Z}+2 \omega^{\prime} \mathbf{Z}\right)$. Thus the dynamical system (3.50) is equivalent to the Lax equation $\dot{L}=[M, L]$, with $L$ and $M N \times N$ matrices given by

$$
\begin{aligned}
L_{i j}(z) & =p_{i} \delta_{i j}+2\left(1-\delta_{i j}\right) \Phi\left(x_{i}-x_{j}, z\right), p_{i}=\dot{x}_{i} \\
M_{i j}(z) & =2 \delta_{i j} \sum_{k \neq i} \wp\left(x_{i}-x_{j}\right)+2\left(1-\delta_{i j}\right) \Phi^{\prime}\left(x_{i}-x_{j}\right)
\end{aligned}
$$

and $\Phi(x, z)$ is the function

$$
\Phi(x, z)=\frac{\sigma(z-x)}{\sigma(z) \sigma(x)} e^{\zeta(z) x}
$$

with $\sigma(z), \zeta(z)$ the usual Weierstrass elliptic functions. In view of the Lax equation, the characteristic polynomial $R(k, z)=\operatorname{det}(2 k+L(z))$ is time-independent, and defines a time-independent spectral curve $\Gamma$

$$
R(k, z) \equiv \sum_{i=0}^{N} r_{i}(z) k^{i}=0,
$$


where the $r_{i}(z)$ are elliptic functions of $z$. The Jacobians of the spectral curves $\Gamma$ are levels of the involutive integrals of the system. In particular, we obtain angle variables $\varphi_{i}$ by choosing $2 \pi$-periodic coordinates on them. However, as noted earlier, the identification of the canonically conjugate action variables is more difficult and has been carried out only recently [29]. In this work, it was shown that the Calogero-Moser sytem can be obtained through a Hamiltonian reduction of a Hitchin system, and as a result, the action-variables $a_{i}$ are the periods of the differential $k d z$ along the $A$-cycles of the spectral curve $\Gamma$.

We can derive this result directly from our approach. From our viewpoint, the leaf $\mathcal{M}$ corresponding to the elliptic Calogero-Moser system is given by $(\Gamma, k, z)$, where $\Gamma$ is a Riemann surface of genus $g=N, k$ is a function with simple poles at $g$ points $P_{1}, \ldots, P_{g}, d z$ is a holomorphic Abelian differential whose periods as well as integrals $\int_{P_{1}}^{P_{\alpha}} d z, 2 \leq \alpha \leq g$, are all on the lattice spanned by $2 \omega$ and $2 \omega^{\prime}$, and the residues of $k d z$ at $P_{\alpha}, 2 \leq \alpha \leq N$ are given by

$$
\operatorname{Res}_{P_{\alpha}}(k d z)=1,2 \leq \alpha \leq N .
$$

The analogues of the Baker-Akhiezer function and its dual are respectively the column vector $C(P)=\left(c_{1}, \ldots, c_{N}\right)$ and the row vector $C^{+}(P)=\left(c_{1}^{+}, \ldots, c_{N}^{+}\right)$, satisfying

$$
\begin{aligned}
(2 k+L(z)) C & =0, \quad C^{+}(2 k+L(z)) & =0, \\
\sum_{i} c_{i} \Phi\left(-x_{i}, z\right) & =1, \quad \sum_{i=1} c_{i}^{+} \Phi\left(x_{i}, z\right) & =1 .
\end{aligned}
$$

Here $P=(k, z)$ is a point of the spectral curve $\Gamma$. The vectors $C(P)$ and $C^{+}(P)$ are meromorphic functions on $\Gamma$ outside the points $P_{\alpha}$ on $\Gamma$ corresponding to $z=0$, and have each $N$ poles. We denote these poles by $\gamma_{1}, \ldots, \gamma_{N}$ and $\gamma_{1}^{+}, \ldots, \gamma_{N}^{+}$, respectively. Near the points $P_{\alpha}$, these vectors have the form

$c_{i}(z)=z\left(N^{-1}+O(z)\right) e^{x_{i} z^{-1}}, \quad c_{i}^{+}(z)=z\left(N^{-1}+O(z)\right) e^{-x_{i} z^{-1}}, \quad$ for $\alpha=1$, $c_{i}(z)=\left(c_{i}^{\alpha}+O(z)\right) e^{x_{i} z^{-1}}, \quad c_{i}^{+}(z)=\left(c_{i}^{\alpha,+}+O(z)\right) e^{-x_{i} z^{-1}}, \quad$ for $\alpha>1$,

where the coefficients $c_{i}^{\alpha}$ for $\alpha>1$ satisfy

$$
\sum_{i} c_{i}^{\alpha}=\sum_{i} c_{i}^{\alpha,+}=0
$$

Let us make the gauge transformation

$$
\tilde{L}=g L g^{-1}, \tilde{C}=g C, \tilde{C}^{+}=C^{+} g^{-1}, g_{i j}=e^{k x_{i}} \delta_{i j} .
$$

The geometric symplectic form $\omega_{\mathcal{M}}$ constructed in Theorem 11 becomes in this case $^{1}$

$$
\omega_{\mathcal{M}}=\delta\left(\sum_{s=1}^{N} k\left(\gamma_{s}\right) d z\right)=\frac{1}{2} \sum_{\alpha=1}^{N} \operatorname{Res}_{P_{\alpha}} \frac{\left\langle\delta \tilde{C}^{+} \wedge \delta \tilde{L} \tilde{C}\right\rangle}{\langle\tilde{C}+\tilde{C}\rangle} d z
$$

\footnotetext{
${ }^{1}$ In [39] the operators $L$ and $C$ in equation (70) should be replaced by their gauge equivalent counterparts $\tilde{L}$ and $\tilde{C}$.
} 
where $\left\langle f^{+} g\right\rangle$ denotes the usual pairing between column vectors and row vectors. Substituting in the expansion (3.56), we obtain

$$
\delta\left(\sum_{s=1}^{N} k\left(\gamma_{s}\right) d z\right)=\frac{1}{2} \sum_{i}^{N} \delta p_{i} \wedge \delta x_{i} .
$$

This identifies our geometric symplectic form with the canonical symplectic form for the Calogero-Moser dynamical system. Since by construction (c.f.(3.42)), the geometric symplectic form admits the periods $a_{i}$ of $k d z$ around $A_{i}$ cycles as action variables dual to the angle variables on the Jacobian, our argument is complete.

\section{Whitham Equations}

\section{A. Non-linear WKB Methods in Soliton Theory}

We have seen that soliton equations exhibit a unique wealth of exact solutions. Nevertheless, it is desirable to enlarge the class of solutions further, to encompass broader data than just rapidly decreasing or quasi-periodic functions. Typical situations arising in practice can involve Heaviside-like boundary conditions in the space variable $x$, or slowly modulated waves which are not exact solutions, but can appear as such over a small scale in both space and time.

The non-linear WKB method (or, as it is now also called, the Whitham method of averaging) is a generalization to the case of partial differential equations of the classical Bogolyubov-Krylov method of averaging. This method is applicable to nonlinear equations which have a moduli space of exact solutions of the form $u_{0}(U x+W t+Z \mid I)$. Here $u_{0}\left(z_{1}, \ldots, z_{g} \mid I\right)$ is a periodic function of the variables $z_{i} ; U=\left(U_{1}, \ldots, U_{g}\right), W=\left(W_{1}, \ldots, W_{g}\right)$ are vectors which like $u$ itself, depend on the parameters $I=\left(I_{1}, \ldots, I_{N}\right)$, i.e. $U=U(I), V=V(I)$. (A helpful example is provided by the solutions (3.10) of the KP equation, where $I$ is the moduli of a Riemann surface, and $U, V, W$ are the $B_{k}$-periods of its normalized differentials $d \Omega_{1}, d \Omega_{2}$, and $d \Omega_{3}$.) These exact solutions can be used as a leading term for the construction of asymptotic solutions

$$
u(x, t)=u_{0}\left(\varepsilon^{-1} S(X, T)+Z(X, T) \mid I(X, T)\right)+\epsilon u_{1}(x, t)+\epsilon^{2} u_{2}(x, t)+\ldots,
$$

where $I$ depend on the slow variables $X=\epsilon x, T=\epsilon t$ and and $\epsilon$ is a small parameter. If the vector-valued function $S(X, T)$ is defined by the equations

$$
\partial_{X} S=U(I(X, T))=U(X, T), \quad \partial_{T} S=W(I(X, T))=W(X, T),
$$

then the leading term of (4.1) satisfies the original equation up to first order one in $\epsilon$. All the other terms of the asymptotic series (4.1) are obtained from the non-homogeneous linear equations with a homogeneous part which is just the linearization of the original non-linear equation on the background of the exact solution $u_{0}$. In general, the asymptotic series becomes unreliable on 
scales of the original variables $x$ and $t$ of order $\epsilon^{-1}$. In order to have a reliable approximation, one needs to require a special dependence of the parameters $I(X, T)$. Geometrically, we note that $\epsilon^{-1} S(X, T)$ agrees to first order with $U x+$ $V y+W t$, and $x, y, t$ are the fast variables. Thus $u(x, t)$ describes a motion which is to first order the original fast periodic motion on the Jacobian, combined with a slow drift on the moduli space of exact solutions. The equations which describe this drift are in general called Whitham equations, although there is no systematic scheme to obtain them.

One approach for obtaining these equations in the case when the original equation is Hamiltonian is to consider the Whitham equations as also Hamiltonian, with the Hamiltonian function being defined by the average of the original one. In the case when the phase dimension $g$ is bigger than one, this approach does not provide a complete set of equations. If the original equation has a number of integrals one may try to get the complete set of equations by averaging all of them. This approach was used in [62] where Whitham equations were postulated for the finite-gap solutions of the $\mathrm{KdV}$ equation. The geometric meaning of these equations was clarified in [26]. The Hamiltonian approach for the Whitham equations of $(1+1)$-dimensional systems was developed in [23] where the corresponding bibliography can also be found.

In [36] a general approach for the construction of Whitham equations for finite-gap solutions of soliton equations was proposed. It is instructive enough to present it in case of the zero curvature equation (2.1) with scalar operators.

Recall from Sections III.A and III.B that the coefficients $u_{i}(x, y, t), v_{j}(x, y, t)$ of the finite-gap operators $L_{0}$ and $A_{0}$ satisfying (2.1) are of the form (c.f. (3.10))

$$
u_{i}=u_{i, 0}(U x+V t+W t+Z \mid I), \quad v_{j}=v_{j, 0}(U x+V t+W t+Z \mid I),
$$

where $u_{i, 0}$ and $v_{j, 0}$ are differential polynomials in $\theta$-functions and $I$ is any coordinate system on the moduli space $\mathcal{M}_{g}(n, m)$.

We would like to construct operator solutions of (2.1) of the form

$$
L=L_{0}+\epsilon L_{1}+\ldots, A=A_{0}+\epsilon A_{1}+\ldots,
$$

where the coefficients of the leading terms have the form

$$
\begin{aligned}
& u_{i}=u_{i, 0}\left(\epsilon^{-1} S(X, Y, T)+Z(X, Y, T) \mid I(X, Y, T)\right), \\
& v_{j}=v_{j, 0}\left(\epsilon^{-1} S(X, Y, T)+Z(X, Y, T) \mid I(X, Y, T)\right)
\end{aligned}
$$

From Section III.B, we also recall that $\mathcal{N}_{g}^{1}(n, m)$ is the bundle over $\mathcal{M}_{g}(n, m)$ with the corresponding curve $\Gamma$ as fiber. If $I$ is a system of coordinates on $\mathcal{M}_{g}(n, m)$, then we may introduce a system of coordinates $(z, I)$ on $\mathcal{N}_{g}^{1}(n, m)$ by choosing a coordinate along the fiber $\Gamma$. The Abelian integrals $p, E, Q$ are multi-valued functions of $(\lambda, I)$, i.e. $p=p(\lambda, I), E=E(\lambda, I), Q=Q(\lambda, I)$. If we describe a drift on the moduli space of exact solutions by a map $(X, Y, T) \rightarrow$ $I=I(X, Y, T)$, then the Abelian integrals $p, E, Q$ become functions of $z, X, Y, T$, 
via

$$
\begin{aligned}
p(z, X, Y, T) & =p(z, I(X, Y, T)), \\
E(z, X, Y, T) & =E(z, I(X, Y, T)) \\
Q(z, X, Y, T) & =Q(z, I(X, Y, T)) .
\end{aligned}
$$

The following was established in [36]:

Theorem 15. A necessary condition for the existence of the asymptotic solution (4.4) with leading term (4.3) and bounded terms $L_{1}$ and $A_{1}$ is that the equation

$$
\frac{\partial p}{\partial z}\left(\frac{\partial E}{\partial T}-\frac{\partial Q}{\partial Y}\right)-\frac{\partial E}{\partial z}\left(\frac{\partial p}{\partial T}-\frac{\partial Q}{\partial X}\right)+\frac{\partial Q}{\partial z}\left(\frac{\partial p}{\partial Y}-\frac{\partial E}{\partial X}\right)=0
$$

be satisfied.

The equation (4.6) is called the Whitham equation for (2.1). It can be viewed as a generalized dynamical system on $\mathcal{M}_{g}(n, m)$, i.e., a map $(X, Y, T) \rightarrow$ $\mathcal{M}_{g}(n, m)$. Some of its important features are:

- Even though the original two-dimensional system may depend on $y$, Whitham solutions which are $Y$-independent are still useful. As we shall see later, this particular case has deep connections with topological field theories. If we choose the local coordinate $z$ along the fiber as $z=E$, then the equation simplifies in this case to

$$
\partial_{T} p=\partial_{X} Q
$$

We note that it followed immediately from the consistency of (4.2) that we must have

$$
\partial_{T} \oint_{B_{k}} d p=\partial_{X} \oint_{B_{k}} d Q
$$

Thus (4.7) is a strengthening of this requirement which encodes also the solvability term by term of the linearized equations for all the successive terms of the asymptotic series (4.3).

- Naively, the Whitham equation seems to impose an infinite set of conditions, since it is required to hold at every point of the fiber $\Gamma$. However, the functions involved are all Abelian integrals, and their equality over the whole of $\Gamma$ can actually be reduced to a finite set of conditions. To illustrate this point, we consider the $Y$-independent Whitham equation on the moduli space of curves of the form

$$
y^{2}=\prod_{i=1}^{3}\left(E-E_{i}\right) \equiv R(E)
$$


Then the differentials $d p$ and $d Q$ are given by

$$
\begin{aligned}
d p & =\frac{d E}{\sqrt{R(E)}}\left(E-\frac{\int_{E_{2}}^{E_{3}} \frac{E d E}{\sqrt{R}}}{\int_{E_{2}}^{E_{3}} \frac{d E}{\sqrt{R}}}\right), \\
d Q & =\frac{d E}{\sqrt{R(E)}}\left(E^{2}-\frac{1}{2}\left(E_{1}+E_{2}+E_{3}\right) E-\frac{\int_{E_{2}}^{E_{3}} \frac{\left(E-\frac{1}{2} \sum_{i=1}^{3} E_{i}\right) E d E}{\sqrt{R}}}{\int_{E_{2}}^{E_{3}} \frac{d E}{\sqrt{R}}}\right) .
\end{aligned}
$$

We view $p$ and $Q$ as functions of $\left(E ; E_{i}\right)$, with $E$ the coordinate in the fiber $\Gamma$, and $E_{i}$ the coordinates on the moduli space of curves. Near each branch point $E_{i}, \sqrt{E-E_{i}}$ is a local coordinate and we may expand

$$
\begin{aligned}
& p=p\left(E_{i}\right)+\alpha \sqrt{E-E_{i}}+O\left(E-E_{i}\right), \\
& Q=Q\left(E_{i}\right)+\beta \sqrt{E-E_{i}}+O\left(E-E_{i}\right) .
\end{aligned}
$$

Differentiating with respect to $X$ and $T$, keeping $E$ fixed, we find that the leading singularities of $\partial_{T} p$ and $\partial_{X} Q$ are respectively $-\frac{\alpha}{2 \sqrt{E-E_{i}}} \partial_{T} E_{i}$ and $-\frac{\beta}{2 \sqrt{E-E_{i}}} \partial_{X} E_{i}$. Since $\frac{\beta}{\alpha}=\frac{d Q}{d p}$, we see that the equation (4.7) implies

$$
\partial_{T} E_{i}=\left.\left(\frac{d Q}{d p}\right)\right|_{E_{i}} \partial_{X} E_{i}
$$

Conversely, if the equation (4.9) is satisfied, then $\partial_{T} p-\partial_{X} Q$ is regular and normalized, and hence must vanish. Thus the equation (4.7) is actually equivalent to the set of differential equations (4.9).

- The equation (4.7) can be represented in a manifestly invariant form, without explicit reference to any local coordinate system $z$. Given a map $(X, Y, T) \rightarrow \mathcal{M}_{g}(n, m)$, the pull back of the bundle $\mathcal{N}_{g}^{1}(n, m)$ defines a bundle over a space with coordinates $X, Y, T$. The total space $\mathcal{N}^{4}$ of the last bundle is 4-dimensional. Let us introduce on it the one-form

$$
\alpha=p d X+E d Y+Q d T,
$$

Then (4.7) is equivalent to the condition that the wedge product of $d \alpha$ with itself be zero (as a 4 -form on $\mathcal{M}^{4}$ )

$$
d \alpha \wedge d \alpha=0 .
$$

- It is instructive to present the Whitham equation (4.7) in yet another form. Because (4.7) is invariant with respect to a change of local coordinate we may use $p=p(z, I)$ by itself as a local coordinate. With this choice we may view $E$ and $Q$ as functions of $p, X, Y$ and $T$, i.e. $E=E(p, X, Y, T), Q=Q(p, X, Y, T)$. With this choice of local coordinate (4.7) takes the form

$$
\partial_{T} E-\partial_{Y} Q+\{E, Q\}=0
$$


where $\{\cdot, \cdot\}$ stands for the usual Poisson bracket of two functions of the variables $p$ and $X$, i.e.

$$
\{f, g\}=f_{p} g_{X}-g_{p} f_{X}
$$

- In Theorem 12 we had focused on constructing an asymptotic solution for a single equation. This corresponds to a choice of $A$, and thus of an Abelian differential $Q$, and the Whitham equation is an equation for maps from $(X, Y, T)$ to $\mathcal{M}_{g}(n, m)$. As in the case of the $\mathrm{KP}$ and other hierarchies, we can also consider a whole hierarchy of Whitham equations. This means that the Abelian integral $Q$ is replaced by the really normalized Abelian integral $\Omega_{i}$ which has the following form

$$
\Omega_{i}=K^{i}+O\left(K^{-1}\right), \quad K^{n}=E,
$$

in a neighborhood of the puncture $P$ (compare with (2.48)). The result is a hierarchy of equations on maps of the form

$$
\left(X=T_{1}, Y=T_{2}, T=T_{3}, T_{4}, \ldots\right) \rightarrow \mathcal{M}_{g}(n) .
$$

The whole hierarchy may be written in the form (4.11) where we set now

$$
\alpha=\sum_{i} \Omega_{i} d T_{i}
$$

\section{B. Exact Solutions of Whitham Equations}

In [38] a construction of exact solutions to the Whitham equations (4.7) was proposed. In the following section, we shall present the most important special case of this construction, which is also of interest to topological field theories and supersymmetric gauge theories. It should be emphasized that for these applications, the definition of the hierarchy should be slightly changed. Namely, the Whitham equations describing modulated waves in soliton theory are equations for Abelian differentials with a real normalization (3.29). In what follows we shall consider the same equations, but where the real-normalized differentials are replaced by differentials with the complex normalization (3.30). As discussed in Section III.A, the two types of normalization coincide on the subspace corresponding to $M$-curves, which is essentially the space where all solutions are regular and where the averaging procedure is easily implemented. Thus the two forms of the Whitham hierarchy can be considered as different extensions of the same hierarchy. The second one is an analytic theory, and we shall henceforth concentrate on it.

In this subsection and in the rest of the paper, we shall restrict ourselves to the hierarchy of "algebraic geometric solutions" of Whitham equations, that is, solutions of the following stronger version of the equations (4.11)

$$
\frac{\partial}{\partial T_{i}} E=\left\{\Omega_{i}, E\right\}
$$


We note that the original Whitham equations can actually be interpreted as consistency conditions for the existence of an $E$ satisfying (4.15). Furthermore, the solutions of $(4.15)$ can be viewed in a sense as " $Y$-independent" solutions of Whitham equations, since the equation (4.12) reduces to $\partial_{T} E+\{E, Q\}=0$ for $Y$-independent solutions. They play the same role as Lax equations in the theory of $(2+1)$-dimensional soliton equations. As stressed earlier, $Y$-independent solutions of the Whitham hierarchy can be considered even for two-dimensional systems where the $y$-dependence is non-trivial in general.

Our first step is to show that (4.15) defines a system of commuting flows on $\mathcal{M}_{g}(n)$. For the sake of simplicity, we assume that there is only one puncture. Let us start with a more detailed description of this space which is a complex manifold with only orbifold singularities. Its complex dimension is equal to (c.f. (3.31)) $\operatorname{dim} \mathcal{M}_{g}(n)=4 g+n-1$, and we had constructed a set of local coordinates for it in Theorem 10 and subsequent discussion. Here we require the following slightly different set of coordinates (details can be found in [38]). The first $2 g$ coordinates are still the periods of $d E$,

$$
\tau_{A_{i}, E}=\oint_{A_{i}} d E, \tau_{B_{i}, E}=\oint_{B_{i}} d E
$$

The differential $d E$ has $2 g+n-1$ zeros (counting multiplicities). When all zeroes are simple, we can supplement (4.16) by the $2 g+n-1$ critical values $E_{s}$ of the Abelian differential $E$, i.e.

$$
E_{s}=E\left(q_{s}\right), \quad d E\left(q_{s}\right)=0, s=1, \ldots, 2 g+n-1,
$$

In general, $d E$ may have multiple zeroes, and we let $D=\sum \mu_{s} q_{s}$ be the zero divisor of $d E$. The degree of this divisor is equal to $\sum_{s} \mu_{s}=2 g-1+n$. Consider a small neighborhood of $q_{s}$, viewed as a point of the fibration $\mathcal{N}_{g}^{1}(n)$, above the original data point $m_{0}$ in the moduli space $\mathcal{M}_{g}(n)$. Viewed as a function on the fibration, $E$ is a deformation of its value $E\left(z, m_{0}\right)$ above the original data point, with multiple critical points $q_{s}$. Therefore, on each of the corresponding curve, there exists a local coordinate $w_{s}$ such that

$$
E=w_{s}^{\mu_{s}+1}(z, m)+\sum_{i=0}^{\mu_{s}-1} E_{s, i}(m) w_{s}^{i}(z, m) .
$$

The coefficients $E_{s, i}(m)$ of the polynomial (4.18) are well-defined functions on $\mathcal{M}_{g}(n)$. Together with $\tau_{A_{i}, E}, \tau_{B_{i}, E}$, they define a system of local coordinates on $\mathcal{M}_{g}(n)$.If $\mu_{s}=1$, then $E_{s, 0}$ clearly coincides with the critical value $E\left(q_{s}\right)$ from (4.17).

Let $\mathcal{D}^{\prime}$ be the open set in $\mathcal{M}_{g}(n)$ where the zero divisors of $d E$ and $d p$, namely the sets $\{z ; d E(z)=0\}$ and $\{z ; d p(z)=0\}$, do not intersect and let $\mathcal{D}^{0}$ be the open set in $\mathcal{M}_{g}(n)$ where all zeros of $d E$ are simple.

Theorem 16. The Whitham equations (4.15) define a system of commuting meromorphic vector fields (flows) on $\mathcal{M}_{g}(n)$ which are holomorphic on 
$\mathcal{D}^{\prime} \subset \mathcal{M}_{g}(n)$. On the open set $\mathcal{D}^{\prime} \cap \mathcal{D}^{0}$, the equations (4.15) have the form

$$
\begin{aligned}
\frac{\partial}{\partial T_{j}} \tau_{A_{i}, E} & =0, \frac{\partial}{\partial T_{j}} \tau_{B_{i}, E}=0 \\
\partial_{T_{j}} E_{s} & =\frac{d \Omega_{j}}{d p}\left(q_{s}\right) \partial_{X} E_{s} .
\end{aligned}
$$

Indeed, the equations (4.15) are fulfilled at each point of $\Gamma$, and thus

$$
\frac{\partial}{\partial T_{i}} \oint_{C} d E=\frac{d \Omega_{i}}{d p}(z) \partial_{X}\left(\oint_{C} d E\right)-\frac{d E}{d p}(z) \partial_{X}\left(\oint_{C} d \Omega_{i}\right)
$$

The functions $d E / d p$ and $d \Omega_{i} / d p$ are linearly independent. It follows that the periods of $d E$ are constants. The equations (4.19) coincide with (4.15) at the point $q_{s}$ (where $d E$ equals zero).

In order to complete the proof of Theorem 13, it suffices to show that (4.184.19) imply (4.15). The equation (4.19) implies that the difference between the left and right hand sides of (4.15) is a meromorphic function $f(z)$ on $\Gamma$. This function is holomorphic outside the puncture $P$ and the zeros of $d p$. At the puncture $P$, the function $f(z)$ has a pole of order less or equal to $(n-2)$. However, $f(z)$ equals zero at zeros of $d E$. Hence, the function $g(z)=f(z) \frac{d p}{d E}$ is holomorphic on $\Gamma$ and equals zero at $P$. Therefore, $f(z)=0$ identically. Theorem 13 is proved.

An important consequence of Theorem 13 is that the space $\mathcal{M}_{g}(n)$ admits a natural foliation, namely by the joint level sets of the functions $\tau_{A_{i}, E}, \tau_{B_{i}, E}$, which are smooth $(2 g+n-1)$-dimensional submanifolds, and which are invariant under the flows of the Whitham hierarchy (4.15). We shall sometimes refer to the leaves $\hat{\mathcal{M}}$ of this foliation as large leaves, to stress their distinction from the $g$-dimensional leaves $\mathcal{M}$ of the canonical foliation of $\mathcal{M}_{g}(n, m)$.

The special case of the construction of exact solutions to (4.15) in [38] may now be described as follows: the moduli space $\mathcal{M}_{g}(n, m)$ provides the solutions of the first $n+m$-flows of (4.15) parametrized by $3 g$ constants, which are the set of the last three coordinates $(3.38-3.39)$ on $\mathcal{M}_{g}(n, m)$.

Theorem 17. Let $T_{i}, i=1, \ldots, n+m, \tau_{A_{i}, E}, \tau_{B_{i}, E}, \tau_{A_{i}, Q}, \tau_{A_{i}, Q}, a_{i}$ be the coordinates on $\mathcal{M}_{g}(n, m)$ defined in Theorem 10. Then the projection

$$
\begin{gathered}
\mathcal{M}_{g}(n, m) \rightarrow \mathcal{M}_{g}(n) \\
(\Gamma, E, Q) \rightarrow(\Gamma, E)
\end{gathered}
$$

defines $(\Gamma, E)$ as a function of the coordinates on $\mathcal{M}_{g}(n, m)$. For each fixed set of parameters $\tau_{A_{i}, E}, \tau_{B_{i}, E}, \tau_{A_{i}, Q}, \tau_{A_{i}, Q}, a_{i}$, the map $\left(T_{i}\right)_{i=1}^{i=n+m} \rightarrow \mathcal{M}_{g}(n)$ satisfies the Whitham equations (4.15).

Proof. Let us use $E(z)$ as a local coordinate on $\Gamma$. Then as we saw earlier, the equations (4.15) are equivalent to the equations $\partial_{T_{i}} p(E, T)=\partial_{X} \Omega_{i}(E, T)$. 
These are the compatibility conditions for the existence of a generating function for all the Abelian differentials $d \Omega_{i}$. In fact, if we set

$$
d \lambda=Q d E
$$

then it follows from the definition of the coordinates that

$$
\partial_{T_{i}} d \lambda=d \Omega_{i}, \partial_{X} d \lambda=d p
$$

(For the proof of (4.23), it is enough to check that the right and the left hand sides of it have the same analytical properties.)

Theorem 18. We consider the same parametrization of $\mathcal{M}_{g}(n, m)$ as in Theorem 14. Then as a function of the parameters $T_{i}, 1 \leq i \leq n+m$, the second Abelian integral $Q(p, T)$ satisfies the same equations as $E$, i.e.

$$
\partial_{T_{i}} Q=\left\{\Omega_{i}, Q\right\}
$$

Furthermore

$$
\{E, Q\}=1 \text {. }
$$

We note that (4.25) can be viewed as a Whitham version of the so-called string equation (or Virasoro constraints) in a non-perturbative theory of 2-d gravity [19][66].

\section{The $\tau$-Function of the Whitham Hierarchy}

The solution of the Whitham hierarchy can be succinctly summarized in a single $\tau$-function defined as follows. The key underlying idea is that suitable submanifolds of $\mathcal{M}_{g}(n, m)$ can be parametrized by $2 g+N-1+\sum_{\alpha=1}^{N}\left(n_{\alpha}+m_{\alpha}\right)$ Whitham times $T_{A}$, to each of which is associated a "dual" time $T_{D A}$, and an Abelian differential $d \Omega_{A}$. We begin by discussing the simpler case of one puncture, $N=1$. Recall that the coefficients of the pole of $d \lambda$ has provided $n+m$ Whitham times $T_{j}=-\frac{1}{j} \operatorname{Res}\left(z^{j} d \lambda\right)$. Their "dual variables" are

$$
T_{D j}=\operatorname{Res}\left(z^{-j} d \lambda\right)
$$

and the associated Abelian differential are the familiar $d \Omega_{i}$ of (3.4) (complex normalized). When $g>0$, the moduli space $\mathcal{M}_{g}(n, m)$ has in addition $5 g$ more parameters. We consider only the foliations for which the following $3 g$ parameters are fixed

$$
\oint_{A_{k}} d E, \oint_{B_{k}} d E, \oint_{A_{k}} d Q
$$

Thus the case $g>0$ leads to two more sets of $g$ Whitham times each

$$
a_{k}=\oint_{A_{k}} d \lambda, T_{k}^{E}=\oint_{B_{k}} d Q
$$


Their dual variables are

$$
a_{D k}=-\frac{1}{2 \pi i} \oint_{B_{k}} d \lambda, T_{D k}^{E}=\frac{1}{2 \pi i} \oint_{A_{k}^{-}} E d \lambda .
$$

The corresponding Abelian differentials are respectively the holomorphic differentials $d \omega_{k}$ and the differentials $d \Omega_{k}^{E}$, defined to be holomorphic everywhere on $\Gamma$ except along the $A_{j}$ cycles, where they have discontinuities

$$
d \Omega_{k}^{E+}-d \Omega_{k}^{E-}=\delta_{j k} d E .
$$

We denote the collection of all $2 g+n+m$ times by $T_{A}=\left(T_{j}, a_{k}, T_{k}^{E}\right)$. In the case of $N>1$ punctures, we have $2 g+\sum_{\alpha}\left(n_{\alpha}+m_{\alpha}\right)$ times $\left(T_{\alpha, j}, a_{k}, T_{k}^{E}\right)$ and $3 N-3$ additional parameters for $\mathcal{M}_{g}(n, m)$, namely the residues of $d Q, d E$, and $d \lambda$ at the punctures $P_{\alpha}, 2 \leq \alpha \leq N$ (c.f. (3.35-3.36)). For simplicity, we only consider the leaves of $\mathcal{M}_{g}(n, m)$ where

$$
\operatorname{Res}_{P_{\alpha}}(d Q)=0, \operatorname{Res}_{P_{\alpha}}(d E)=\text { fixed, } 2 \leq \alpha \leq N
$$

and incorporate among the $T_{A}$ the residues of $d \lambda$ at $P_{\alpha}, 2 \leq \alpha \leq N, R_{\alpha}^{\lambda}=$ $\operatorname{Res}_{P_{\alpha}}(d \lambda)$ (c.f.(3.35)). The dual parameters to these $N-1$ additional Whitham times are then the regularized integrals

$$
R_{D \alpha}^{\lambda}=-\int_{P_{1}}^{P_{\alpha}} d \lambda, 2 \leq \alpha \leq N .
$$

More precisely, recall that the Abelian integral $\lambda$ has been fixed by the condition that its expansion near $P_{1}$, in terms of the local coordinate $z_{\alpha}$ defined by $E$, have no constant term. Near each $P_{\alpha}, 2 \leq \alpha \leq N$, in view of (4.31), it admits an expansion of the form

$$
\lambda\left(z_{\alpha}\right)=\sum_{j=1}^{n_{\alpha}+m_{\alpha}} \frac{T_{\alpha, j}}{z_{\alpha}^{j}}+\operatorname{Res}_{P_{\alpha}}(d \lambda) \log z_{\alpha}+\lambda_{\alpha}+O\left(z_{\alpha}\right) .
$$

For each $\alpha, 2 \leq \alpha \leq N$, we define the right hand side of (4.32) to be $\lambda_{\alpha}$. The Abelian differential $d \Omega_{\alpha}^{(3)}$ associated with $R_{\alpha}^{\lambda}$ is the Abelian differential of the third kind, with simple poles at $P_{1}$ and $P_{\alpha}$, and residue 1 at $P_{\alpha}$. In summary, we have the following table

\section{Times Dual Times Differentials}

$$
\begin{array}{ccc}
-\frac{1}{j} \operatorname{Res}_{P_{\alpha}}\left(z^{j} d \lambda\right) & \operatorname{Res}_{P_{\alpha}}\left(z^{-j} d \lambda\right) & d \Omega_{\alpha, j}^{0} \\
\oint_{A_{k}} d \lambda & -\frac{1}{2 \pi i} \oint_{B_{k}} d \lambda & d \omega_{k} \\
\operatorname{Res}_{P_{a}} d \lambda & -\int_{P_{1}}^{P_{\alpha}} d \lambda & d \Omega_{\alpha}^{(3)} \\
\oint_{B_{k}} d Q & \frac{1}{2 \pi i} \oint_{A_{k}} E d \lambda & d \Omega_{k}^{E}
\end{array}
$$


We can now define the $\tau$-function of the Whitham hierarchy by

$$
\begin{aligned}
\tau(T) & =e^{\mathcal{F}(T)}, \\
\mathcal{F}(T) & =\frac{1}{2} \sum_{A} T_{A} T_{D A}+\frac{1}{4 \pi i} \sum_{k=1}^{g} a_{k} T_{k}^{E} E\left(A_{k} \cap B_{k}\right),
\end{aligned}
$$

where $A_{k} \cap B_{k}$ is the point of intersection of the $A_{k}$ and $B_{k}$ cycles. In the case $\operatorname{Res}_{P_{a}} d E=0$ we have then (see [38])

Theorem 19. The derivatives of $\mathcal{F}$ with respect to the $2 g+\sum_{\alpha}\left(n_{\alpha}+m_{\alpha}\right)+N-1$ Whitham times $T_{A}$ are given by

$$
\begin{aligned}
\partial_{T_{A}} \mathcal{F} & =T_{D A}+\frac{1}{2 \pi i} \sum_{k=1}^{g} \delta_{a_{k}, A} T_{k}^{E} E\left(A_{k} \cap B_{k}\right), \\
\partial_{T_{\alpha, i}, T_{\beta, j}}^{2} \mathcal{F} & =\operatorname{Res}_{P_{\alpha}}\left(z_{\alpha}^{i} d \Omega_{\beta, j}\right), \\
\partial_{a_{j}, A}^{2} \mathcal{F} & =\frac{1}{2 \pi i}\left(E\left(A_{k} \cap B_{k}\right) \delta_{(E, k), A}-\oint_{B_{k}} d \Omega_{A}\right), \\
\partial_{(E, k) A}^{2} \mathcal{F} & =\frac{1}{2 \pi i} \oint_{A_{k}} E d \Omega_{A}, \\
\partial_{A B C}^{3} \mathcal{F} & =\sum_{q_{s}} \operatorname{Res}_{q_{s}}\left(\frac{d \Omega_{A} d \Omega_{B} d \Omega_{C}}{d E d Q}\right) .
\end{aligned}
$$

These formulae require some modifications when $\operatorname{Res}_{P_{\alpha}} d E \neq 0$, which is a case of particular interest in supersymmetric QCD (see Section VI below). In particular, the first derivatives with respect to $R_{\alpha}^{\lambda}$ become [17]

$$
\partial_{R_{\alpha}^{\lambda}} \mathcal{F}=T_{D \alpha}^{\lambda}+\frac{1}{2} \pi i \sum_{\beta} c_{\alpha, \beta} R_{\beta}^{\lambda}
$$

where $c_{\alpha, \beta}$ is an integer which is antisymmetric in $\alpha$ and $\beta$. It is then easy to see that the second derivatives are modified accordingly by constant shifts, while the third derivatives remain unchanged.

We would like to point out that the $2 g+\sum_{\alpha}\left(n_{\alpha}+m_{\alpha}\right)+N-1$ submanifolds of $\mathcal{M}_{g}(n, m)$ defined by fixing (4.27) as well as the residues of $d E$ and $d Q$ are yet another version of the $2 g+n-1$ large leaf $\hat{\mathcal{M}}$ of the foliation of $\mathcal{M}_{g}(n)$ encountered earlier in the case of one puncture. Indeed, imbedding $\mathcal{M}_{g}(n)$ in $\mathcal{M}_{g}(n, 1)$ by choosing $Q_{+}=k$ would fix two Whitham times $T_{n}$ and $T_{n+1}$, as we saw after Theorem 10. This reduces the dimension $2 g+n+1$ to the desired dimension $2 g+n-1$.

We observe that the first derivatives of $\mathcal{F}$ give the coefficients of the Laurent expansions and the periods of the form $\lambda$. The second derivatives give the coefficients of the expansions and the periods of the differentials $d \Omega_{A}$. In that sense the function $\mathcal{F}$ encodes all the information on the Whitham hierarchy. The 
formulae for the first and the second derivatives with respect to the variables $T_{i}$ are the analogues in the case of the averaged equations of the corresponding formulae for the $\tau$-function of the KP hierarchy. The formulae for the third derivatives are specific to the Whitham theory. As we shall see later, they are reminiscent rather of marginal deformations of topological or conformal field theories and of special geometry.

Finally, it may be worth noting that the expression (4.35) for $\mathcal{F}$ can be elegantly summarized as

$$
\mathcal{F}=\int_{\Gamma} \bar{d} \lambda \wedge d \lambda
$$

\section{Topological Landau-Ginzburg Models on a Ri- amman Surface}

In this section, we shall show that each $2 g+n-1$ leaf of the foliation of $\mathcal{M}_{g}(n)$ (or, equivalently, of the foliation of $\mathcal{M}_{g}(n, 1)$ upon imbedding $\mathcal{M}_{g}(n)$ into $\mathcal{M}_{g}(n, 1)$ ) actually parametrizes the marginal deformations of a topological field theory on a surface of genus $g$. Furthermore, the free energy of these theories coincides with the restriction to the leaf of the exponential of the $\tau$ function for the Whitham hierarchy. We begin with a brief discussion of some key features of topological field theories [63-65][11-13].

\section{A. Topological Field Theories}

In general, a two-dimensional quantum field theory is specified by the correlation functions $\left\langle\phi\left(z_{1}\right) \ldots \phi\left(z_{N}\right)\right\rangle_{g}$ of its local physical observables $\phi_{i}(z)$ on any surface $\Gamma$ of genus $g$. Here $\phi_{i}(z)$ are operator-valued tensors on $\Gamma$. The operators act on a Hilbert space of states with a designated vacuum state $|\Omega\rangle$. The correlators $\left\langle\phi\left(z_{1}\right) \ldots \phi\left(z_{N}\right)\right\rangle$ usually depend on the background metric on $\Gamma$ and on the location of the insertion points $z_{i}$. In particular, they may develop singularities as $z_{i}$ approaches $z_{j}$. Equivalently, the operator product $\phi_{i}\left(z_{i}\right) \phi_{j}\left(z_{j}\right)$ may develop singularities. For example, in a conformal field theory, $\phi_{i}\left(z_{i}\right) \phi_{j}\left(z_{j}\right)$ will have an operator product expansion of the form

$$
\phi_{i}\left(z_{i}\right) \phi_{j}\left(z_{j}\right)=\sum_{k} c_{i j}^{k}\left(z_{i}-z_{j}\right)^{h_{i}+h_{j}-h_{k}} \phi_{k}\left(z_{j}\right)+\text { descendants }
$$

where $h_{i}$ is the conformal dimension of $\phi_{i}$. If we let $\phi_{0}(z)$ be the field corresponding to $|\Omega\rangle$, under the usual states $\leftrightarrow$ fields correspondence, then we obtain a metric by setting

$$
\eta_{i j}=c_{i j}^{0} .
$$

Using $\eta_{i j}$ to raise and lower indices, and noting that $\left\langle\phi_{0}(z)\right\rangle_{0}=1$, we can easily recognize $c_{i j k}=c_{i j}^{k} \eta_{k l}$ as the three-point function on the sphere

$$
c_{i j k}=\left\langle\phi_{i}\left(z_{i}\right) \phi_{j}\left(z_{j}\right) \phi_{k}\left(z_{k}\right)\right\rangle_{0},
$$


which is actually independent of the insertion points $z_{i}, z_{j}, z_{k}$ by $\operatorname{SL}(2, \mathbf{C})$ invariance.

Topological field theories are theories where the correlation functions are actually independent of the insertion points $z_{i}$. Thus they depend only on the labels of the fields $\phi_{i}$ and the genus $g$ of $\Gamma$. This independence implies that the OPE of (5.1) contains no singularity, and that for all practical purposes, the operator product $\phi_{i}\left(z_{i}\right) \phi_{j}\left(z_{j}\right)$ can be replaced by the formal operator algebra

$$
\phi_{i} \phi_{j}=\sum_{k} c_{i j}^{k} \phi_{k}
$$

The associativity of operator compositions translates into the associativity of the operator algebra (5.4). Furthermore, the operator algebra is commutative, since factorization of the 4-point function in the $s$ and $t$ channels must give the same answer. If we assume that in a topological field theory, physical correlation functions can be factored through only physical states (as is the case, if the Hilbert space of physical states arises as the cohomology of a nilpotent BRST operator $Q$ acting on a larger Hilbert space containing spurious states), then the correlation functions of the theory on surfaces of all genera can all be expressed explicitly, through factorization, in terms of the structure constants $c_{i j k}$.

\section{B. Deformations of a Topological Field Theory}

We shall be particularly interested in the case where the topological field theory arises as a deformation by parameters $t_{i}$ of a fixed theory. In a topological field theory, the physical fields $\phi_{i}$ have clearly scaling dimension 0 . In presence of a BRST symmetry, they actually fit in a multiplet $\Phi_{i}=\left(\phi_{i}^{(0)}=\phi_{i}, \phi_{i}^{(1)}, \phi_{i}^{(2)}\right)$, where the "descendants" $\phi_{i}^{(d)}$ are tensors of scaling dimension $d$ which satisfy the descent equations $d \phi^{(d)}=\left\{Q, \phi^{(d+1)}\right\}$. In particular, $\phi_{i}^{(2)}$ can be integrated on the surface $\Gamma$. If we let $I(0)$ be the Lagrangian of the original theory, we can deform the theory to another theory with Lagrangian

$$
I(t)=I(0)-\sum_{i} t_{i} \int_{\Gamma} \phi_{i}^{(2)}
$$

The structure constants $c_{i j k}(t)$ of the new theory are then given by

$$
c_{i j k}(t)=\left\langle\phi_{i} \phi_{j} \phi_{k} \exp \left(\sum_{i} t_{i} \int_{\Gamma} \phi_{i}^{(2)}\right)\right\rangle_{0} .
$$

They define in turn a topological field theory. For deformations around a topological conformal field theory, i.e., a topological field theory whose stress tensor is already traceless before restricting to physical states, the structure constants $c_{i j k}(t)$ are known to satisfy the key compatibility condition $\partial_{l} c_{i j k}=\partial_{k} c_{i j l}$ 
[11-13]. This means that we can find a function $\mathcal{F}$, called the free energy and formally denoted by

$$
\mathcal{F}(t)=\left\langle\exp \left(\sum_{i} t_{i} \int \Phi_{i}\right)\right\rangle_{0}
$$

which satisfies the third derivative condition

$$
\partial_{i j k}^{3} \mathcal{F}(t)=c_{i j k} .
$$

In terms of $\mathcal{F}$, the commutativity and associativity conditions of the operator algebra with structure constants $c_{i j k}(t)$ become a system of differential equations of third order, called the Witten-Dijkgraaf-Verlinde-Verlinde (WDVV) equation.

\section{The Framework of Solitons}

In their original work [11-12], Dijkgraaf, Verlinde, and Verlinde derived an explicit expression for the free energy $\mathcal{F}(t)$ in the case of topological LandauGinzburg theories. These are the topological theories arising from twisting an $\mathrm{N}=2$ superconformal field theory, which is itself obtained by following the renormalization group flow to the fixed point of a Landau-Ginzburg model. Although the renormalization group flow modifies the kinetic terms in the Landau-Ginzburg action, the superpotential $W(x)$ remains unchanged, and thus characterizes both the associated superconformal and the topological models [30][61][67]. Our goal in this section is to show how this theory can fit in the framework of solitons, and to exhibit the natural emergence of the differential $Q d E$ and Whitham times.

We consider first the case of genus 0 , with $\Gamma=\{z \in \mathbf{C} \cup \infty\}$. The role of the superpotential $W(x)$ is played in our context by the Abelian integral $E$ with a unique pole of order $n$ at $\infty$. We consider then a leaf in the space $\mathcal{M}_{0}(n, 1)$, characterized by the condition that $Q=z$, and $E$ is of the form

$$
E=z^{n}+\sum_{i=0}^{n-2} u_{i} z^{i}+O\left(z^{-1}\right)
$$

This is of dimension $n-1$, and can be parametrized by the $n-1$ Whitham times $T_{A}, A=1, \ldots, n-1$, with the other times fixed to $T_{n}=0, T_{n+1}=\frac{n}{n+1}$. At each point $E$ on the leaf, the primary fields $\phi_{i}$ can be identified with $d \Omega_{i} / d Q$. The structure constants are defined by

$$
\left\langle\phi_{i} \phi_{j}\right\rangle=\operatorname{Res}_{\infty}\left(\frac{d \Omega_{(i)} d \Omega_{(j)}}{d E}\right),\left\langle\phi_{i} \phi_{j} \phi_{k}\right\rangle=\operatorname{Res}_{\infty}\left(\frac{d \Omega_{(i)} d \Omega_{(j)} d \Omega_{(k)}}{d E d Q}\right) .
$$

We note that $d Q$ corresponds to the field defined by the vacuum. The following can be derived from Theorem 16: 
Theorem 20. Let $\mathcal{F}(T)$ be the $\tau$-function of the (complex normalized) Whitham hierarchy, restricted to the $(n-1)$ dimensional leaf described above. Then

(i) the fields $\phi_{i}$ anti-diagonalize the pairing $\eta_{i j}$, i.e., $\left\langle\phi_{i} \phi_{j}\right\rangle=\delta_{i+j, n}$;

(ii) $\mathcal{F}(T)$ is the free energy of the theory, i.e., $\partial_{T_{i} T_{j} T_{k}}^{3} \mathcal{F}(T)=c_{i j k}$. In particular, $\mathcal{F}(T)$ satisfies the system of WDVV equations.

More generally, the case of $\Gamma$ of genus $g$ (with one puncture to simplify the notation) has been treated in [22][38]. In this case, the relevant leaf within $\mathcal{M}_{g}(n, 1)$ is of dimension $n-1+2 g$ and is given by the constraints

$$
\begin{aligned}
& T_{n}=0, T_{n+1}=\frac{n}{n+1} \\
& \oint_{A_{k}} d E=0, \oint_{B_{k}} d E=\text { fixed } \\
& \oint_{A_{k}} d Q=0 .
\end{aligned}
$$

Thus the leaf is parametrized by the $(n-1)$ Whitham times $T_{A}, A=1, \ldots, n-1$, and by the periods $a_{j}$ and $T_{j}^{E}$ of (4.28). The fields $\phi_{A}$ of the theory need to be augmented accordingly. We take the $2 g$ additional ones to be given by $d \omega_{j} / d Q$ and $d \Omega_{j}^{E} / d Q$, where the differentials $d \omega_{j}$ and $d \Omega_{j}^{E}$ are the ones associated to $a_{j}$ and $T_{j}^{E}$, as described earlier in (4.30).

Theorem 21. Let $\eta_{A B}$ and $c_{A B C}$ be defined as

$$
\begin{gathered}
\eta_{A, B}=\sum_{q_{s}} \operatorname{Res}_{q_{s}} \frac{d \Omega_{A} d \Omega_{B}}{d E}, \\
c_{A B C}=\sum_{q_{s}} \operatorname{Res}_{q_{s}} \frac{d \Omega_{A} d \Omega_{B} d \Omega_{C}}{d E d Q},
\end{gathered}
$$

with $q_{s}$ the zeroes of $d E$, and the indices $A, B, C$ running this time through the augmented set of $n-1+2 g$ indices given by $T_{A}=\left(T_{i}, a_{j}, T_{E, j}\right)$. Then

(i) $\eta_{i, j}=\delta_{i+j, n}, \eta_{a_{j},(E, k)}=\delta_{j, k}$. All other pairings vanish;

(ii) Let $\mathcal{F}\left(T_{i}, a_{j}, T_{E, j}\right)$ be the $\tau$-function of the Whitham hierarchy restricted to the leaf (5.11). Then $\partial_{A B C}^{3} \mathcal{F}(T)=c_{A B C}$, where $A$ runs through the augmented set of $n-1+2 g$ indices.

Note that in the genus 0 case when $Q=z$, the sum in (5.12-5.13) over the residues at the zeros of $d E$ reduces to the residue at infinity.

Remarkably, the larger spaces $\mathcal{M}_{g}(n, m)$ can accommodate the gravitational descendants of the fields $\phi_{A}$. More precisely, consider for $g=0$ the leaf of the space $\mathcal{M}_{0}(n, m n+1)$ given by the following evident modification of the earlier normalization (5.11)

$$
T_{i n}=0, i=1, \ldots, m, \quad T_{n m+1}=\frac{n m}{n m+1} .
$$


The space of Whitham times is automatically increased to the correct number by taking all the coefficients of $Q d E$. The additional $m(n-1)$ fields may be identified with the first $m$ gravitational descendants of the primary fields. Namely, the $p$-th descendant $\sigma_{p}\left(\phi_{i}\right)$ of the primary field $\phi_{i}$ is just $\frac{d \Omega_{p n+i}}{d Q}$. This statement is a direct corollary of the following theorem.

Theorem 22. The correlation functions given by $\left\langle\phi_{A} \phi_{B} \phi_{C}\right\rangle=\partial_{A B C}^{3} \mathcal{F}$ with $\sigma_{p}\left(\phi_{i}\right)=d \Omega_{i+p n} / d Q$ satisfy the factorization properties for descendant fields

$$
\left\langle\sigma_{p}\left(\phi_{i}\right) \phi_{B} \phi_{C}\right\rangle=\left\langle\sigma_{p-1}\left(\phi_{i}\right) \phi_{j}\right\rangle \eta^{j k}\left\langle\phi_{k} \phi_{B} \phi_{C}\right\rangle,
$$

where $\phi_{i}, i=1, \ldots, n-1$ are primary fields, and $\phi_{A}$ are all fields (including descendants).

Factorization properties for descendant fields were derived by Witten [63$66]$. We note that the completeness of the operator algebra requires a larger set of fields that just $g$, which is the dimension of the small leaves of the canonical foliation of $\mathcal{M}_{g}(n, m)$, and which will be shown in the next section to be the dimension of the moduli space of vacua of certain supersymmetric gauge theories. This is one of the difficulties in establishing direct contact between topological field theories and supersymmetric gauge theories, although there has been progress in this direction [6][44][45].

\section{Seiberg-Witten Solutions of $\mathrm{N}=2$ Supersym- metric Gauge Theories}

Moduli spaces of geometric structures are appearing increasingly frequently as the key to the physics of certain supersymmetric gauge or string theories. One recurrent feature is a moduli space of degenerate vacua in the physical theory. The physics of the theory is then encoded in a Kähler geometry on the space of vacua, or, in presence of powerful constraints such as $\mathrm{N}=2$ supersymmetry, in an even more restrictive special geometry, where the Kähler potential is dictated by single holomorphic function $\mathcal{F}$, called the prepotential. This was the case for Type IIA and Type IIB strings, where the vacua corresponding to compactifications on Calabi-Yau threefolds [31][68] produce effective $\mathrm{N}=2$ four dimensional supergravity theories. The massless scalars of such theories (in this case, the moduli of the Calabi-Yau threefold) must parametrize a manifold equipped with special geometry [9][51][57]. More recently, a similar phenomenon has been brought to light by Seiberg-Witten [52][53] for $\mathrm{N}=2$ supersymmetric gauge theories. Remarkably, the space of vacua of these theories, which is classically just a space of diagonalizable and traceless matrices, becomes upon quantization a moduli space of Riemann surfaces. The prepotential $\mathcal{F}$ for the quantum effective theory can then be derived from a meromorphic one-form $d \lambda$ on each surface. A particularly striking feature of these effective theories, noticed by many authors $[24][28][42-43][49][56]$, is a strong but as yet ill-understood similarity with the 
Whitham theory of solitons. Indeed, the quantum spaces of vacua for many $\mathrm{N}=2$ SUSY theories actually coincide with certain leaves of the canonical foliation of $\mathcal{M}_{g}(n, m)$ [39], the Seiberg-Witten form $d \lambda$ with the one-form $Q d E$ central to Whitham theory, and the effective prepotential of the gauge theories with the exponential of the $\tau$-function of the Whitham theory! The purpose of this section is to review some of these developments.

\section{A. $N=2$ Supersymmetric Gauge Theories}

We begin with a brief account of $\mathrm{N}=2$ SUSY Yang-Mills theories in four dimensions with gauge group $G$ [2]. The Yang-Mills gauge field $A=A_{\mu} d x^{\mu}$ is imbedded in an $\mathrm{N}=2$ gauge multiplet consisting of $A$, left and right Weyl spinors $\lambda_{L}$ and $\lambda_{R}$, and a complex scalar field $\phi$, with all fields valued in the adjoint representation of $G$. The requirement of $\mathrm{N}=2$ SUSY and renormalizability fixes uniquely the action

$$
I=\int_{M^{4}} d^{4} x \operatorname{Tr}\left[\frac{1}{4 g^{2}} F \wedge F^{*}+\frac{\theta}{8 \pi^{2}} F \wedge F+D \phi^{\dagger} \wedge * D \phi+\left[\phi, \phi^{\dagger}\right]^{2}\right]+\text { fermions },
$$

where $g$ is the coupling constant, $\theta$ is the instanton angle, and we have written explicitly only the bosonic part of the action. The classical vacua are given by the critical points of the action. In this case, they work out to be $A=0, \phi$ is constant (up to a gauge transformation), and

$$
\left[\phi, \phi^{\dagger}\right]=0 \text {. }
$$

Thus $\phi$ must lie in the Cartan subalgebra. For $G=\operatorname{SU}\left(N_{c}\right)\left(N_{c}\right.$ is commonly referred to as number of "colors"), we set

$$
\phi=\left(\begin{array}{ccccc}
\bar{a}_{1} & & & & \\
& \bar{a}_{2} & & & \\
& & \ddots & & \\
& & & \ddots & \\
& & & & \bar{a}_{N_{c}}
\end{array}\right), \sum_{k=1}^{N_{c}} \bar{a}_{k}=0 .
$$

Thus the classical space of vacua is parametrized by the $\vec{a}_{k}$, up to a Weyl permutation.

For a generic configuration $\bar{a}_{k}$, we have $\bar{a}_{j} \neq \bar{a}_{k}$ for any $j \neq k$, and the gauge group $\mathrm{SU}\left(N_{c}\right)$ is spontaneously broken down to $\mathrm{U}(1)^{N_{c}-1}$. At the quantum level, we expect then the space of inequivalent vacua to be parametrized by $N_{c}-1$ parameters $a_{k}$ (thought of as renormalizations of the $\bar{a}_{k}, \sum_{k=1}^{N_{c}} a_{k}=0$ ), with each vacuum corresponding to a theory of $N_{c}-1$ interacting $\mathrm{U}(1)$ gauge fields $A_{j}$, i.e., $N_{c}-1$ copies of electromagnetism. In the weak coupling regime, we expect singularities at $a_{k}=a_{l}$, where the gauge symmetry is suddenly enhanced. Since $\mathrm{N}=2 \mathrm{SUSY}$ remains unbroken, each gauge field $A_{j}$ is part of an $\mathrm{N}=2 \operatorname{SUSY} \mathrm{U}(1)$ gauge multiplet $\left(A_{j}, \lambda_{L j}, \lambda_{R j}, \phi_{j}\right)$, all in the adjoint 
representation of $U(1)$. Again, the action for a theory with such a field content is fixed by $\mathrm{N}=2$ supersymmetry. To leading order in the low momentum expansion for these fields, it must be of the form

$$
I_{\text {eff }}=\frac{1}{8 \pi} \int_{M^{4}} d^{4} x\left[\left(\operatorname{Im} \tau^{j k}\right) F_{j} \wedge * F_{k}+\left(\operatorname{Re} \tau^{j k}\right) F_{j} \wedge F_{k}+d \phi^{\dagger i} \wedge d \phi_{D}^{i}\right]+\text { fermions }
$$

where

$$
\tau^{j k}=\frac{\partial^{2} \mathcal{F}}{\partial a_{j} \partial a_{k}}, \phi_{D}^{j}=\frac{\partial \mathcal{F}}{\partial a_{j}}(\phi)
$$

for a suitable complex and analytic function $\mathcal{F}(a, \Lambda)$, called the prepotential. We note that the prepotential $\mathcal{F}$ is a function not just of the vacua parameters $a_{k}$, but also of a scale $\Lambda$ introduced by renormalization.

Thus the physics of the quantum theory is encoded in a single function $\mathcal{F}$. What is known about $\mathcal{F}$ ? To insure the positivity of the kinetic energy, we must have

$$
\operatorname{Im} \frac{\partial^{2} \mathcal{F}}{\partial a_{j} \partial a_{k}}>0
$$

Geometrically, $\mathcal{F}$ defines then a Kähler metric on the quantum moduli space by

$$
d s^{2}=\sum \operatorname{Im}\left(\frac{\partial^{2} \mathcal{F}}{\partial a_{j} \partial a_{k}}\right) d a_{j} \overline{d a_{k}}
$$

Furthermore, at weak-coupling $\Lambda \ll 1, \mathcal{F}$ can be evaluated in perturbation theory. For pure $\mathrm{SU}\left(N_{c}\right)$ Yang-Mills, one finds

$$
\mathcal{F}(a, \Lambda)=\frac{2 N_{c}}{2 \pi i} \sum_{k=1}^{N_{c}} a_{k}^{2}-\frac{1}{8 \pi i} \sum_{j, k=1}^{N_{c}}\left(a_{k}-a_{j}\right)^{2} \log \frac{\left(a_{k}-a_{j}\right)^{2}}{\Lambda^{2}}+\sum_{d=1}^{\infty} \mathcal{F}_{d} \Lambda^{2 N_{c} d}
$$

The first term on the right hand side is the classical prepotential. The second term is the perturbative one-loop quantum correction. In view of $\mathrm{N}=2$ nonrenormalization theorems, it is known that higher loops do not contribute. The third term is the instanton contribution, consisting of $d$-instanton processes for all $d$. We observe that the expansion (6.8) implies in particular that $\mathcal{F}$ has nontrivial monodromy around $a_{j}=a_{k}$ in the $\Lambda \ll 1$ regime. The exact solution of $\mathrm{N}=2$ Yang-Mills theories is reduced in this way to finding a holomorphic $\mathcal{F}$ satisfying the constraints (6.6) and (6.8).

We have just described the main problem for $\mathrm{N}=2$ SUSY pure $\mathrm{SU}(N)$ YangMills theories. However, the same problem should be addressed for general $\mathrm{N}=2$ SUSY gauge theories with gauge group $\mathrm{G}$, with matter fields ("hypermultiplets") in a representation $R$ of $G$. As in the case of pure Yang-Mills, the Wilson effective Lagrangian of these theories is dictated by a prepotential $\mathcal{F}_{G, R}(a, \Lambda)$, and the problem is to determine $\mathcal{F}_{G, R}(a, \Lambda)$, subject to the constraints (6.6) and (6.8), where the right hand side of (6.8) has been modified to incorporate the contributions of the hypermultiplets. For example, in presence 
of $N_{f}$ hypermultiplets in the fundamental representation of bare masses $m_{i}$, $1 \leq i \leq N_{f}$, the one-loop correction to the prepotential for the $\mathrm{SU}\left(N_{c}\right)$ theory contains the additional term

$$
\sum_{k=1}^{N_{c}} \sum_{i=1}^{N_{f}}\left(a_{k}+m_{j}\right)^{2} \log \frac{\left(a_{k}+m_{j}\right)^{2}}{\Lambda^{2}} .
$$

\section{B. The Seiberg-Witten Ansatz}

The requirements that $\mathcal{F}$ have monodromy and a Hessian with positive definite imaginary part, suggest an underlying non-trivial geometry on the quantum space of vacua. In [52][53], Seiberg and Witten made the fundamental Ansatz that

- For each $\Lambda$, the quantum moduli space should parametrize a family of Riemann surfaces $\Gamma(a, \Lambda)$ of genus $g=N_{c}-1$, now known as the spectral curves of the theory;

- on each $\Gamma(a, \Lambda)$, there is a meromorphic one-form $d \lambda$;

- $\mathcal{F}$ is determined by the periods of $d \lambda^{2}$

$$
a_{k}=\frac{1}{2 \pi i} \oint_{A_{k}} d \lambda, \quad a_{D, k}=\frac{1}{2 \pi i} \oint_{B_{k}} d \lambda, \quad \frac{\partial \mathcal{F}}{\partial a_{k}}=a_{D, k} .
$$

The gauge theories under consideration contain dyons, i.e., particles which carry both electric and magnetic charges. Let $(n, m) \in \mathbf{Z}^{N_{c}-1} \times \mathbf{Z}^{N_{c}-1}$ be their charges, with $\left(n_{i}, m_{i}\right)$ the charge with respect to the $i$-th $\mathrm{U}(1)$ factor. The $\mathrm{N}=2$ SUSY algebra implies the bound $M^{2} \geq 2\left|a n+a_{D} m\right|^{2}$ from below for their masses. Thus the states saturating this bound, known as Bogomolny-PrasadSommerfeld or BPS states, are described by the lattice spanned by the periods of $d \lambda$. The singular locus of the fibration $\Gamma(a, \Lambda)$, namely the points where the curve degenerates and a period $a_{j}$ or $a_{D, j}$ vanishes, corresponds then to vacua where one or several dyons become massless.

For pure $\mathrm{SU}(2)$ Yang-Mills, the monodromy prescription at $\infty$ is restrictive enough to suggest the identification of the quantum moduli space of vacua with $H / \Gamma(2)$ ( $H$ denotes the upper half space, and $\Gamma(2)$ the subgroup of $\operatorname{SL}(2, \mathbf{Z})$ matrices congruent to 1 mod 2), assuming the minimal number two of singularities in the interior of the quantum moduli space. Since then, spectral curves have been proposed for a variety of gauge theories with matter, based on physical considerations such as decoupling, or analogies with singularity theory or soliton theory (see e.g. [40] and references therein). However, at the present

\footnotetext{
${ }^{2}$ In this section, we adopt the normalization (6.9) for the periods $a_{i}$ of $d \lambda$ rather than (3.39), in keeping with the literature on Seiberg-Witten theory. Similarly, the present $\mathcal{F}$ differs from the earlier $\tau$-function $\mathcal{F}_{\text {Whitham }}$ of soliton theory (c.f. $(4.35)$ ) by $\mathcal{F}=-\frac{1}{2 \pi i} \mathcal{F}_{\text {Whitham }}$.
} 
time, we still do not have a complete correspondence between the group theoretic characterization of the gauge theory, consisting of the group $G$ and the representation $R$ for the hypermultiplets, and the fibration of spectral curves which characterizes its geometric and physical content.

\section{The Framework of the Theory of Solitons}

Nevertheless, an intriguing feature of most of the spectral curves for $\mathrm{N}=2$ SUSY gauge theories known so far, is that they, together with the one-form $d \lambda$, fit exactly in the framework of the foliation on $\mathcal{M}_{g}(n, m)$ with $d \lambda=Q d E$. In particular,

- The spectral curves for $\mathrm{SU}\left(N_{c}\right)$ theories with $N_{f}<2 N_{c}$ hypermultiplets in the fundamental representation of bare masses $m_{i}, 1 \leq i \leq N_{f}$, are given by the leaf $(\Gamma, E, Q)$ with the following properties.

- $d E$ has simple poles, at points $P_{+}, P_{-}, P_{i}$, with residues $-N_{c}, N_{c}-N_{f}$, and $1\left(1 \leq i \leq N_{f}\right)$ respectively. Its periods around homology cycles are integer multiples of $2 \pi i$;

- $Q$ is a well-defined meromorphic function, with simple poles only at $P_{+}$ and $P_{-}$;

- The other parameters of the leaf are fixed by the following normalization of the one-form $d \lambda=Q d E$

$$
\begin{aligned}
\operatorname{Res}_{P_{i}}(d \lambda) & =-m_{i}, \\
\operatorname{Res}_{P_{+}}(z d \lambda) & =-N_{c} 2^{-1 / N_{c}}, \operatorname{Res}_{P_{-}}(z d \lambda) \\
& =\left(N_{c}-N_{f}\right)\left(\frac{\Lambda^{2 N_{c}-N_{f}}}{2}\right)^{1 /\left(N_{c}-N_{f}\right)} \\
\operatorname{Res}_{P_{+}}(d \lambda) & =0 .
\end{aligned}
$$

Here $z=E^{-1 / N_{c}}$ or $z=E^{1 /\left(N_{c}-N_{f}\right)}$ is as usual the holomorphic coordinate system near $P_{+}$or near $P_{-}$adapted to the Abelian integral $E$.

These conditions imply that $\Gamma$ is hyperelliptic, and admits an equation of the form (see [39])

$$
y^{2}=\prod_{k=1}^{N_{c}}\left(Q-\bar{a}_{k}\right)^{2}-\Lambda^{2 N_{c}-N_{f}} \prod_{j=1}^{N_{f}}\left(Q+m_{j}\right) \equiv A(Q)^{2}-B(Q) .
$$

Strictly speaking, the parameters $\bar{a}_{k}$ of (6.11) agree with the classical vacua in (6.3) only when $N_{c}<N_{f}$. For $N_{f} \geq N_{c}$, there are $O(\Lambda)$ corrections, which can be absorbed in a reparametrization leaving the prepotential $\mathcal{F}$ invariant [15]. Thus we may view the $\bar{a}_{k}$ of (6.3) and (6.11) as identical. If we represent the Riemann surface (6.11) by a two-sheeted covering of the complex plane, 
then the meromorphic function $Q$ on $\Gamma$ in $d \lambda=Q d E$ is just the coordinate in each sheet, while the Abelian integral $E$ is given by $E=\log (y+A(Q))$. The points $P_{ \pm}$correspond to the points at infinity, with the two possible choices of signs \pm for $y= \pm \sqrt{A^{2}-B}$. To choose a canonical homology basis, we let $x_{k}^{ \pm}$ $1 \leq k \leq N_{c}$, be the branch points $A\left(x_{k}^{ \pm}\right)^{2}-B\left(x_{k}^{ \pm}\right), A_{k}$ be a simple contour enclosing the slit from $x_{k}^{-}$to $x_{k}^{+}$for $2 \leq k \leq N_{c}$, and $B_{k}$ be the curve going from $x_{k}^{-}$to $x_{1}^{-}$on each sheet. We can now give a preliminary and easy check that the curve (6.11) is consistent with the expected behavior of the theory at weak-coupling. Consider for simplicity the case of pure Yang-Mills, $N_{f}=0$. Then as $\Lambda \rightarrow 0$, the discriminant of the curve behaves as $\Lambda^{N_{c}} \prod_{j<k}\left(\bar{a}_{j}-\bar{a}_{k}\right)^{2}$, and the singularities are at the expected location. Furthermore, in this limit, $d \lambda \sim Q \frac{A^{\prime}(Q)}{A(Q)} d Q+\ldots$, and the cycles $A_{k}$ are just contours in the complex plane around a slit which shrinks to a single point $\bar{a}_{k}$. The residue formula gives at once

$$
a_{k}=\frac{1}{2 \pi i} \oint_{A_{k}} Q \frac{A^{\prime}(Q)}{A(Q)} d Q+O\left(\Lambda^{N_{c}}\right)=\bar{a}_{k}+O\left(\Lambda^{N_{c}}\right),
$$

identifying $\bar{a}_{k}$ as a classical order parameter.

- The spectral curves for the other classical gauge groups with matter in the fundamental representation are restrictions of the ones for $\mathrm{SU}\left(N_{c}\right)$ [4][16];

- The $\mathrm{SU}\left(N_{c}\right)$ theory with matter in the adjoint representation is of particular interest. For massless matter, the theory has actually an $\mathrm{N}=4$ supersymmetry, and is conformally invariant. As the hypermultiplet acquires mass, the $\mathrm{N}=4$ SUSY is broken down to an $\mathrm{N}=2 \mathrm{SUSY}$. In [18], Donagi and Witten argued that the spectral curves for the theory are then given by Hitchin systems. Expressed in terms of elliptic Calogero-Moser systems, the curves they proposed are given precisely by the leaf $(\Gamma, k, z)$ in Section III.D. Here the hypermultiplet mass has been scaled to 1 , and the moduli $\tau=\omega_{2} / \omega_{1}$ of the torus is the microscopic gauge coupling.

Although this suggests a deep relation between $\mathrm{N}=2$ gauge theories and integrable models, such a relation is still not fully understood at the present time. Nevertheless, the parallelism between the two fields allows us to apply to the study of the prepotential $\mathcal{F}$ of gauge theories the methods developed in the theory of solitons. Thus Theorem 16 implies readily [17]

Theorem 23. The prepotential $\mathcal{F}$ for $\mathrm{SU}\left(N_{c}\right)$ gauge theories with $N_{f}<2 N_{c}$ hypermultiplets of masses $m_{j}$ in the fundamental representation, satisfies the following differential equation

$$
\begin{aligned}
\sum_{j=1}^{N_{c}} a_{j} & \frac{\partial \mathcal{F}}{\partial a_{j}}+\sum_{j=1}^{N_{f}} m_{j} \frac{\partial \mathcal{F}}{\partial m_{j}}-2 \mathcal{F}= \\
& -\frac{1}{2 \pi i}\left[\operatorname{Res}_{P_{+}}(z d \lambda) \operatorname{Res}_{P_{+}}\left(z^{-1} d \lambda\right)+\operatorname{Res}_{P_{-}}(z d \lambda) \operatorname{Res}_{P_{-}}\left(z^{-1} d \lambda\right)\right]
\end{aligned}
$$


We observe that there is a slight abuse of language here, since in the case of the effective prepotential of gauge theories, $\mathcal{F}$ is only fixed up to $a_{k}$ independent terms by (6.9). This is consistent with the fact that only derivatives of $\mathcal{F}$ with respect to $a_{k}$ occur in the effective action. Thus the $a_{k}$ independent terms on the right hand side of $(6.13)$ can be ignored by adjusting $\mathcal{F}$. The prepotential (4.35) (restricted to the leaf corresponding to the $\mathrm{SU}\left(N_{c}\right)$ theory) is one choice of $\mathcal{F}$. Another choice suggested by dimensional analysis (c.f. (6.8)) is the prepotential $\mathcal{F}$ satisfying the homogeneity condition $\left(\frac{\partial}{\partial \Lambda}+\mathcal{D}\right) \mathcal{F}=0$. In this case, we recognize (6.13) as a renormalization group equation, with the beta function given by the right hand side of (6.13). Earlier versions of (6.13) appear in [24][46][47][56].

To illustrate the power of Theorem 20, we shall show how it can generate explicit expressions for the contributions of instanton processes to any order. Thus we consider the regime where $\Lambda$ is small and all the $A_{k}$-cycles degenerate simultaneously. A fundamental observation is that in this regime, the quantum order parameters $a_{k}$ are perturbations of their classical counterparts $\tilde{a}_{k}$ which can be determined explicitly to any order. In fact, as noted in the arguments leading to (6.12), the $A_{k}$ cycles are simple contours shrinking to a point in one sheet of the Riemann surface and residue formulae apply. The approximation (6.12) can be improved to

$$
\begin{aligned}
& a_{k}=\bar{a}_{k}+\sum_{m=1}^{\infty} \frac{\Lambda^{\left(2 N_{c}-N_{f}\right) m}}{2^{2 m}(m !)^{2}}\left(\frac{\partial}{\partial \bar{a}_{k}}\right)^{2 m-1} \bar{S}_{k}\left(\bar{a}_{k}\right), \\
& \bar{S}_{k}(x)=\frac{\prod_{j=1}^{N_{f}}\left(x+m_{j}\right)}{\prod_{l \neq k}\left(x-\bar{a}_{l}\right)^{2}} .
\end{aligned}
$$

The evaluation of the dual periods $a_{D k}$ is of course more difficult. We need to show that the prepotential $\mathcal{F}$, as defined by the $B_{k}$-periods, reproduces the classical prepotential $\mathcal{F}^{(0)}$ in (6.8) (with hypermultiplets) and satisfies the nonrenormalization theorem. This requires an analytic continuation in an auxiliary parameter $\xi$, as explained in [15]. However, once this is established, the difficult instanton contributions can be derived from the renormalization group equation. Setting $\mathcal{F}=\mathcal{F}^{(0)}+\mathcal{F}^{(1)}+\mathcal{F}^{(2)}+\ldots$, we have, say up to 2 -instanton order and using Euler's homogeneity relation,

$$
\sum_{j=1}^{N_{c}} a_{j} \frac{\partial \mathcal{F}}{\partial a_{j}}+\sum_{j=1}^{N_{f}} m_{j} \frac{\partial \mathcal{F}}{\partial m_{j}}=\left(N_{f}-2 N_{c}\right)\left(\frac{1}{4 \pi i} \sum_{k=1}^{N_{c}} a_{k}^{2}+\mathcal{F}^{(1)}+2 \mathcal{F}^{(2)}\right) \text {. }
$$

(We note the overall factor $N_{f}-2 N_{c}$, which confirms the known conformal invariance of the theory with $N_{f}=2 N_{c}$. For the spectral curves of this theory, we refer to [4]). On the other hand, dropping all $a_{k}$-independent terms, the right hand side of (6.13) is easily found

$$
\sum_{j=1}^{N_{c}} a_{j} \frac{\partial \mathcal{F}}{\partial a_{j}}+\sum_{j=1}^{N_{f}} m_{j} \frac{\partial \mathcal{F}}{\partial m_{j}}-2 \mathcal{F}=\frac{1}{4 \pi i}\left(N_{f}-2 N_{c}\right) \sum_{k=1}^{N_{c}} \bar{a}_{k}^{2}
$$


We can now use (6.14) to reexpress the right hand side of (6.16) in terms of the quantum order parameters $a_{k}$. The instanton contributions can then be read off after suitable rearrangements [15][17]

$$
\begin{aligned}
& \mathcal{F}^{(1)}=\frac{1}{8 \pi i} \Lambda^{2 N_{c}-N_{f}} \sum_{k=1}^{N_{c}} S_{k}\left(a_{k}\right) \\
& \mathcal{F}^{(2)}=\frac{1}{32 \pi i} \Lambda^{2\left(2 N_{c}-N_{f}\right)}\left(\sum_{k \neq l} \frac{S_{k}\left(a_{k}\right) S_{l}\left(a_{l}\right)}{\left(a_{k}-a_{l}\right)^{2}}+\frac{1}{4} \sum_{k=1}^{N_{c}} S_{k}\left(a_{k}\right) \partial_{a_{k}}^{2} S_{k}\left(a_{k}\right)\right) .
\end{aligned}
$$

Here the function $S_{k}(x)$ is defined in analogy with (6.14) by

$$
S_{k}(x)=\frac{\prod_{j=1}^{N_{f}}\left(x+m_{j}\right)}{\prod_{j \neq k}^{N_{c}}\left(x-a_{j}\right)^{2}}
$$

We turn next to a determination of the effective prepotential at strong coupling. In general, when a single cycle $A_{k}$ or $B_{k}$ degenerates, we expect the effective prepotential to be expressible in terms of functions on the resulting surface of lower genus. A particularly interesting case is the behavior of $\mathcal{F}$ near a point on the quantum moduli space of maximum degeneracy, where all $B_{k}$ cycles degenerate simultaneously, and the spectral curve degenerates to two spheres connected by thin tubes. Physically, this means that a maximum number of mutually local dyons become simultaneously massless. As shown in [20], the points of maximum degeneracy occur at the curves $y^{2}=A_{0}(Q)^{2}-4 \Lambda^{2 N_{c}}$, where $A_{0}(Q)$ is given by the $N$-th Chebyshev polynomial

$$
A_{0}=2 \Lambda^{N_{c}} C_{0}\left(\frac{Q}{2 \Lambda}\right), C_{0}(z)=\cos (N \arccos (z))
$$

A neighborhood of the maximum degeneracy point on the quantum moduli space is parametrized by polynomials $P(Q)$ of degree $N-2$, with the spectral curve $y^{2}=A(Q)^{2}-4 \Lambda^{2 N_{c}}, A(Q)=A_{0}(Q)+2 \Lambda^{N_{c}} P\left(\frac{Q}{2 \Lambda}\right)$. Since it is the $B_{k}$ cycle which degenerates this time, it is more convenient to express the prepotential $\mathcal{F}\left(a_{D k}\right)$ and the beta function in terms of the dual variables $a_{D k}$, which can then be evaluated to an arbitrary order of accuracy by residue methods. The renormalization group equation remains the same under interchange of the dual variables $a_{k} \leftrightarrow a_{D k}$

$$
\sum_{k=1}^{N_{c}} a_{D k} \frac{\partial \mathcal{F}}{\partial a_{D k}}-2 \mathcal{F}=\frac{2 N_{c}}{2 \pi i} u\left(a_{D}\right)
$$

where $u$ is the coefficient of $Q^{N_{c}-2}$ in $A(Q)$. Residue calculations show next 
$a_{D k}$ is of first order in $P$ and that

$$
\begin{aligned}
a_{D k} & =i(-)^{k} \frac{s_{k}}{N_{c}} P\left(c_{k}\right)+\sum_{m=1}^{\infty} a_{D k}^{(m)} \\
s_{k} & =\sin \left(\frac{k \pi}{N_{c}}\right), c_{k}=\cos \left(\frac{k \pi}{N_{c}}\right), k=1, \ldots, N_{c}
\end{aligned}
$$

where the $a_{D k}^{(m)}$ are of order $O^{m}\left(a_{D}\right)$ and can be evaluated explicitly. To third order in $a_{D k}$, we find for $u$

$$
u=2 i \Lambda \sum_{k=1}^{N_{c}-1} s_{k} a_{D k}+\frac{1}{4 N_{c}} \sum_{k=1}^{N_{c}-1} a_{D k}^{2}+\frac{i}{32 N_{c}^{2} \Lambda}\left[\sum_{k=1}^{N_{c}-1} \frac{a_{D k}^{2}}{s_{k}^{3}}-4 \sum_{k \neq l}^{N_{c}-1} \frac{a_{D k}^{2} a_{D l} s_{l}}{\left(c_{k}-c_{l}\right)^{2}}\right]
$$

Solving the renormalization group equation, we obtain [14]

Theorem 24. Near the point of maximum degeneracy on the quantum moduli space given by (6.19), the prepotential $\mathcal{F}$ is given by the following expression

$$
\begin{aligned}
\mathcal{F}\left(a_{D}\right)=-\frac{2 N_{c} \Lambda}{\pi} \sum_{k=1}^{N_{c}-1} s_{k} a_{D k} & -\frac{i}{4 \pi} \sum_{k=1}^{N_{c}-1} a_{D k}^{2} \log \frac{a_{D k}}{\Lambda_{k}} \\
+ & \frac{1}{32 \pi N_{c} \Lambda}\left[\sum_{k=1}^{N_{c}-1} \frac{a_{D k}^{3}}{s_{k}^{3}}-4 \sum_{k \neq l}^{N_{c}-1} \frac{a_{D k}^{2} a_{D l} s_{l}}{\left(c_{k}-c_{l}\right)^{2}}\right]
\end{aligned}
$$

up to third order in the order parameters $a_{D k}$. Here $\Lambda_{k}$ is determined by $\log \frac{\Lambda_{k}}{\Lambda}=\frac{3}{2}+\log s_{k}$.

We should mention that there is by now an extensive literature on SeibergWitten theories, and we refer to [40] for a description of other recent advances and for a more complete list of references.

\section{Bibliography}

[1] Adler, M., On a trace functional for formal pseudo-differential operators and the symplectic structure for Korteweg-de Vries type equations, Invent. Math. 50 (1979) 219-248.

[2] Amati, D., Konishi, K., Y. Meurice, G.C. Rossi, and G. Veneziano, Nonperturbative aspects of supersymmetric gauge theories, Physics Reports 162 (1988) 169-248.

[3] Arbarello, E. and C. DeConcini, On a set of equations characterizing Riemann matrices, Ann. of Math. 120 (1984) 119-140. 
[4] Argyres, P. and A.P. Shapere, The vacuum structure of $N=2$ super-QCD with classical gauge groups, Nucl. Phys. B 461 (1996) 437-459, hepth/9508175.

[5] Bogoyavlenski, O. and S.P. Novikov, On the connection between Hamiltonian formalisms of stationary and non-stationary problems, Funct. Anal. and Appl. 10 (1976) 9-13.

[6] G. Bonelli and M. Matone, Non-perturbative relations in N=2 SUSY YangMills and WDVV equations, Phys.Rev.Lett. 77 (1996) 4712-4715, hepth/9605090.

[7] Cherednik, I., Differential equations for the Baker-Akhiezer functions of algebraic curves, Funct. Anal. and Appl. 12 (1978) 45-52.

[8] Date, E., M. Jimbo, M. Kashiwara, and T. Miwa, Transformation groups for soliton equations, in M. Jimbo and T. Miwa (eds) Non-linear integrable systems-classical and quantum theory, Proc. RIMS Symposium, Singapore, 1983.

[9] de Wit, B., P.G. Lauwers, and A. van Proeyen, Lagrangians of $N=2$ supergravity-matter systems, Nucl. Phys. B 255 (1985) 569-608.

[10] Dickey, L.A., Soliton equations and Hamiltonian systems, Advanced Series in Mathematical Physics, Vol. 12 (1991) World Scientific, Singapore.

[11] Dijkgraaf, R., E. Verlinde, and H. Verlinde, Topological strings in $d<1$, Nucl. Phys. B 352 (1991) 59-86.

[12] Dijkgraaf, R., E. Verlinde, and H. Verlinde, Notes on topological string theory and 2D quantum gravity, in String theory and quantum gravity, Proceedings of the Trieste Spring School 1990, M. Green (ed), WorldScientific, 1991.

[13] Dijkgraaf, R. and E. Witten, Mean field theory, topological field theory, and multi-matrix models, Nucl. Phys. B 342 (1990) 486-522.

[14] D'Hoker, E. and D.H. Phong, Strong coupling expansions in $S U\left(N_{c}\right)$ Seiberg-Witten theory, Phys. Lett. B 397 (1997) 94-103.

[15] D'Hoker, E., I.M. Krichever, and D.H. Phong, The effective prepotential for $N=2$ supersymmetric $S U(N)$ gauge theories, Nucl. Phys. B 489 (1997) 179-210.

[16] D'Hoker, E., I.M. Krichever, and D.H. Phong, The effective prepotential for $N=2$ supersymmetric $S O(N)$ and $S p(2 N)$ gauge theories, Nucl. Phys. B 489 (1997) 211-222. 
[17] D'Hoker, E., I.M. Krichever, and D.H. Phong, The renormalization group equation for $N=2$ supersymmetric gauge theories, Nucl. Phys. B494 (1997) 89-104, hep-th/9610156.

[18] Donagi, R. and E. Witten, Supersymmetric Yang-Mills theory and integrable systems, Nucl. Phys. B 460 (1996) 299-334, hep-th/9610101.

[19] Douglas, M. and S. Shenker, Strings in less than one dimension, Nucl. Phys. B 335 (1990) 635-654.

[20] Douglas, M. and S. Shenker, Dynamics of SU(3) gauge theories, Nucl. Phys. B 447 (1995) 271-296, hep-th/9503163.

[21] Dubrovin, B.A., Integrable systems in topological field theory, Nucl. Phys. B 379 (1992) 627-689.

[22] Dubrovin, B.A., Hamiltonian formalism of Whitham-type hierarchies and topological Landau-Ginzburg models, Comm. Math. Phys. 145 (1992) 195207.

[23] Dubrovin, B.A. and S.P. Novikov, The Hamiltonian formalism of onedimensional systems of the hydrodynamic type, and the BogoliubovWhitham averaging method, Sov. Math. Doklady 27 (1983) 665-669.

[24] Eguchi, T. and S.K. Yang, Prepotentials of $N=2$ supersymmetric gauge theories and soliton equations, Mod.Phys.Lett. A 11 (1996) 131-138, hepth/9510183.

[25] Faddeev, L. and L. Takhtajan, Hamiltonian methods in the theory of soliton, Springer-Verlag, 1987.

[26] Flaschka, H., Forrest, M.G., and D. McLaughlin, Multiphase averaging and the inverse spectral solution of the Korteweg-de Vries equation, Comm. Pure Appl. Math. 33 (1980) 739-784.

[27] Gelfand, I.M. and L.A. Dickey, Fractional powers of operators and Hamiltonian systems, Funct. Anal. and Appl. 10 (1976) 13-29.

[28] Gorsky, A., I.M. Krichever, A. Marshakov, A. Mironov, and A. Morozov, Phys. Lett. B 355 (1995) 466-474.

[29] Gorsky, A. and N. Nekrasov, Elliptic Calogero-Moser system from twodimensional current algebra, preprint ITEP-NG/1-94, hep-th/9401021.

[30] Greene, B., C. Vafa, and N. Warner, Calabi-Yau manifolds and renormalization group flows, Nucl. Phys. B 324 (1989) 371-390.

[31] Greene, B. and S.T. Yau, eds., Mirror Symmetry II, Studies in Advanced Mathematics, American Mathematical Society-International Press (1997), Rhode Island and Hong Kong. 
[32] Klemm, A., W. Lerche, and S. Theisen, Non-perturbative actions of $N=2$ supersymmetric gauge theories, Int. J. Mod. Phys. A 11 (1996) 1929-1974, hep-th/9505150.

[33] Krichever, I.M., The algebraic-geometric construction of Zakharov-Shabat equations and their solutions, Doklady Akad. Nauka USSR 227 (1976) 291-294.

[34] Krichever, I.M., Methods of algebraic geometry in the theory of non-linear equations, Russian Math Surveys 32 (1977) 185-213.

[35] Krichever, I.M., Elliptic solutions of the Kadomtsev-Petviashvili equation and integrable systems of particles, Funct. Anal. Appl. 14 (1980) 282-290.

[36] Krichever, I.M., Averaging method for two-dimensional integrable equations, Funct. Anal. Appl. 22 (1988) 37-52.

[37] Krichever, I.M., Spectral theory of two-dimensional periodic operators and its applications, Russian Math. Surveys (1989) 44 (1989) 145-225.

[38] Krichever, I.M., The $\tau$-function of the universal Whitham hierarchy, matrix models, and topological field theories, Comm. Pure Appl. Math. 47 (1994) 437-475.

[39] Krichever, I.M. and D.H. Phong, On the integrable geometry of soliton equations and $N=2$ supersymmetric gauge theories, J. Differential Geometry 45 (1997) 349-389.

[40] Lerche, W., Introduction to Seiberg-Witten theory and its stringy origins, Proceedings of the Spring School and Workshop in String Theory, ICTP, Trieste (1996), hep-th/9611190.

[41] Magri, F., A simple model of the integrable Hamiltonian equation, J. Math. Phys. 19 (1978) 1156-1162.

[42] Martinec, E., Integrable structures in supersymmetric gauge and string theory, Phys. Lett. B 367 (1996) 91-96, hep-th/9510204.

[43] Martinec, E. and N. Warner, Integrable models and supersymmetric gauge theory, Nucl. Phys. B 459 (1996) 97-112, hep-th/9609161.

[44] Marshakov, A., Non-perturbative quantum theories and integrable equations, Int. J. Mod. Phys. A 12 (1997) 1607-1650, hep-th/9610242.

[45] Marshakov, A., A. Mironov, and A. Morozov, WDVV-like equations in N=2 SUSY Yang-Mills theory, hep-th/9607109.

[46] Matone, M., Instantons and recursion relations in N=2 Susy gauge theory, Phys. Lett. B 357 (1995) 342-348. 
[47] Minahan, J. and D. Nemeschansky, N=2 Super Yang-Mills and Subgroups of $S L(2, Z)$, Nucl. Phys. B 468 (1996) 73-84, hep-th/9601059.

[48] Mumford, D., Theta Functions I, II, Birkhäuser (1980) Boston.

[49] Nakatsu, T. and K. Takasaki, Whitham-Toda hierarchy and N=2 supersymmetric Yang-Mills theory, Mod. Phys. Lett. A 11 (1996) 157-168, hep-th/9509162.

[50] Novikov, S.P. and A. Veselov, On Poisson brackets compatible with algebraic geometry and Korteweg-deVries dynamics on the space of finite-zone potentials, Soviet Math. Doklady 26 (1982) 357-362.

[51] Periwal, V. and A. Strominger, Kähler geometry of the space of $N=2$ supersonformal field theories, Phys. Lett. B 235 (1990) 261-267.

[52] Seiberg, N. and E. Witten, Electric-magnetic duality, Monopole Condensation, and Confinement in N=2 Supersymmetric Yang-Mills Theory, Nucl. Phys. B 426 (1994) 1952, hep-th/9407087.

[53] Seiberg, N. and E. Witten, Monopoles, Duality and Chiral Symmetry Breaking in N=2 Supersymmetric QCD, Nucl. Phys. B 431 (1994) 484550 , hep-th/9410167.

[54] Sato, M., Soliton equations and the universal Grassmann manifold, Math. Notes. Series 18 (1984) Sophia University, Tokyo.

[55] Shiota, T., Characterization of Jacobian varieties in terms of soliton equations, Invent. Math. 83 (1986) 333-382.

[56] Sonnenschein, J., S. Theisen, and S. Yankielowicz, On the relations between the holomorphic prepotential and the quantum moduli in SUSY gauge theories, Phys. Lett. B 367 (1996) 145-150, hep-th/9510129.

[57] Strominger, A., Special Geometry, Comm. Math. Phys. 133 (1990) 163180.

[58] Takebe, T., Representation theoretic meaning for the initial-value problem for the Toda lattice hirearchy, Lett. Math. Phys. 21 (1991), 77-84.

[59] Terng, C.L., Soliton equations and differential geometry, J. Differential Geometry 45 (1997) 407-445.

[60] Terng, C.L. and K. Uhlenbeck, to appear.

[61] Vafa, C., Topological Landau-Ginzburg models, Mod. Phys. Lett. A 6 (1991) 337-346.

[62] Whitham, G., Linear and non-linear waves, Wiley (1974) New York. 
[63] Witten, E., Topological Quantum Field Theory, Comm. Math. Phys. 117 (1988) 353-386.

[64] Witten, E., Topological sigma models, Comm. Math. Phys. 118 (1988) 411-449.

[65] Witten, E., Mirror manifolds and topological field theory, in Essays on Mirror Manifolds, ed. by S.T. Yau, International Press (1992), Hong-Kong, 120-159.

[66] Witten, E., Two-dimensional gravity and intersection theory on moduli space, Surveys in Differential Geometry 1 (1991) 281-332.

[67] Witten, E., Phases of $N=2$ theories in two dimensions, Nucl. Phys. B 403 (1993) 159-222; also reprinted in [31].

[68] Yau, S.T., ed., Essays on Mirror Manifolds, International Press (1992) Hong-Kong. 
